\title{
Ammonia as an Ultimate Amino Source in the Synthesis of Primary Amines via Nickel-Promoted C-H Bond Amination
}

Lin Yu, ${ }^{a}$ Chan Yang, ${ }^{a}$ Yongqi Yu, ${ }^{a}$ Da Liu,,${ }^{a}$ Liang $\mathrm{Hu},{ }^{a}$ Yuanjiu Xiao, ${ }^{\mathrm{a}}$ Ze-Nan Song, ${ }^{\mathrm{a}}$ and Ze Tan ${ }^{\mathrm{a}, *}$

a State Key Laboratory of Chemo/Biosensing and Chemometrics, College of Chemistry and Chemical Engineering, Hunan University, Changsha 410082, P. R. China.

e-mail:ztanze@gmail.com

\section{Supporting Information}

\section{Table of contents}

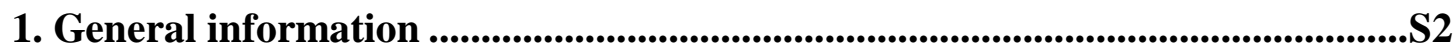

2. Typical procedure for the preparation of aryl amides...........................................S2

3. Nickel-promoted $\mathrm{C}$-H amination to form primary anilines .................................S4

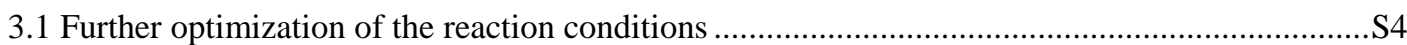

3.2 General procedure for nickel-promoted $\mathrm{C}-\mathrm{H}$ amination to form primary anilines......................S5

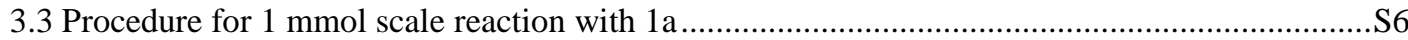

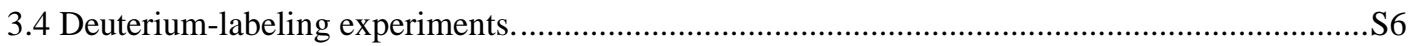

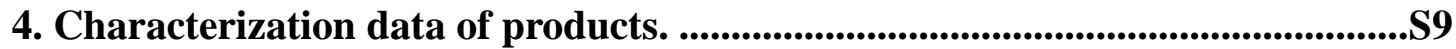

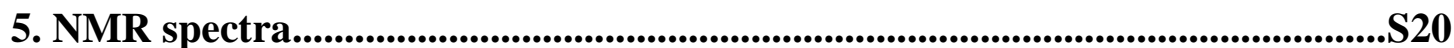




\section{General information}

${ }^{1} \mathrm{H}$ NMR and ${ }^{13} \mathrm{C}$ NMR were recorded in $\mathrm{CDCl}_{3}$ or DMSO- $d_{6}$ at room temperature on the Varian INOVA-400 spectrometer $\left(400 \mathrm{MHz}{ }^{1} \mathrm{H}\right)$ or Bruker spectrometer (400 $\left.\mathrm{MHz}{ }^{1} \mathrm{H}\right)$. The chemical-shifts scale is based on internal TMS. The peak patterns are indicated as follows: s, singlet; d, doublet; t, triplet; q, quartet; m, multiplet; qui, quin tet; sxt, sextet. The coupling constants, $J$ are reported in Hertz (Hz). Mass spectros -copy data were collected on an HRMS-ESI instrument.

Unless otherwise noted, all reagents were obtained from commercial suppliers and used without further purification. Anhydrous $\mathrm{NiCl}_{2}$ was purchased from Alfa Aesar. All solvents were purified and dried according to standard methods prior to use. Products were purified by flash column chromatography on 100-160 mesh silica gel, $\mathrm{SiO}_{2}$.

\section{Typical procedure for the preparation of aryl amides}

All amides 1 were synthesized from the corresponding benzoic acids or benzoyl chlorides and aromatic amine. The deuterated amides were synthesized according to a literature method, spectral properties are consistent with literature values. ${ }^{1}$ The amides $\mathbf{1 a b}-\mathbf{1 v}$ were synthesized according to literature procedures. ${ }^{2}$<smiles>O=C(Nc1ccccc1C1=NCCO1)c1ccccc1</smiles><smiles>CC(C)(NC(=O)c1ccccc1)c1ccccn1</smiles><smiles>COc1ccccc1NC(=O)c1ccccc1</smiles><smiles>O=C(Nc1cccc[n+]1[O-])c1ccccc1</smiles><smiles>O=C(NCc1ccccc1)c1ccccn1</smiles><smiles>O=C(Nc1cccc2cccnc12)c1ccccc1</smiles><smiles>Cc1ccc(C(=O)Nc2cccc3cccnc23)cc1</smiles>

$1 b$<smiles>COc1ccc(C(=O)Nc2cccc3cccnc23)cc1</smiles><smiles>C=Cc1ccc(C(=O)Nc2cccc3cccnc23)cc1</smiles> 
<smiles>O=C(Nc1cccc2cccnc12)c1ccc(-c2ccccc2)cc1</smiles><smiles>N#Cc1ccc(C(=O)Nc2cccc3cccnc23)cc1</smiles><smiles>COC(=O)c1ccc(C(=O)Nc2cccc3cccnc23)cc1</smiles><smiles>O=C(Nc1cccc2cccnc12)c1ccc(OC(F)(F)F)cc1</smiles><smiles>O=C(Nc1cccc2cccnc12)c1ccc(C(F)(F)F)cc1</smiles><smiles>O=C(Nc1cccc2cccnc12)c1ccc(F)cc1</smiles>

$1 \mathrm{~h}$

1 i<smiles>O=C(Nc1cccc2cccnc12)c1ccc(Cl)cc1</smiles><smiles>O=C(Nc1cccc2cccnc12)c1ccc(Br)cc1</smiles><smiles>O=C(Nc1cccc2cccnc12)c1ccc([N+](=O)[O-])cc1</smiles><smiles>O=C(Nc1cccc2cccnc12)c1cccc(-c2ccccc2)c1</smiles><smiles>O=C(Nc1cccc2cccnc12)c1cccc(C(F)(F)F)c1</smiles><smiles>CC(=O)c1cccc(C(=O)Nc2cccc3cccnc23)c1</smiles><smiles>O=C(Nc1cccc2cccnc12)c1ccccc1C(F)(F)F</smiles>

$1 q$<smiles>O=C(Nc1cccc2cccnc12)c1cccc2ccccc12</smiles>
$1 r$<smiles>O=C(Nc1cccc2cccnc12)c1ccncc1</smiles><smiles>CCCCNc1cccc2cccnc12</smiles><smiles>O=C(Nc1cccc2cccnc12)c1ccc(Cl)c(Cl)c1</smiles><smiles>O=C(Nc1cccc2cccnc12)C1=CCCCC1</smiles><smiles>O=C(Nc1cccc2cccnc12)c1ccc(-c2ccccn2)cc1</smiles><smiles>[X]c1ccc(-n2cccn2)cc1C(=O)Nc1cccc2cccnc12</smiles>

Procedure for the synthesis of substrates $\mathbf{1 w}$ and $\mathbf{1} \mathbf{x}^{3}$. 


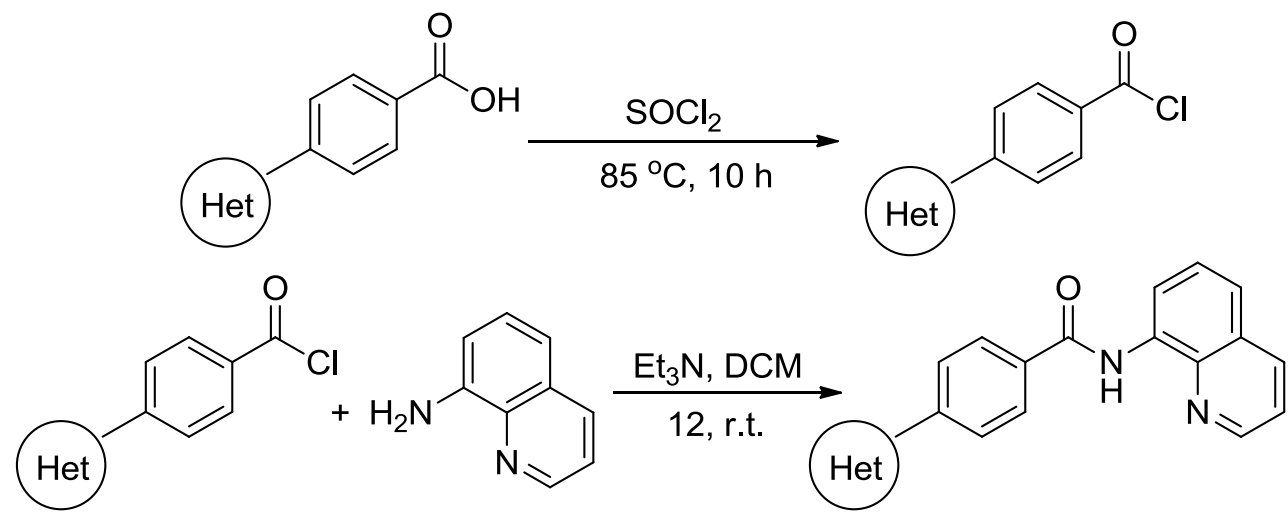

An acid chloride $(5 \mathrm{mmol})$, prepared from the corresponding carboxylic acid and thionyl chloride and quinolin- 8 -amine $(5 \mathrm{mmol})$ were added to a $50 \mathrm{~mL}$ flask and then dissolved with DCM $(20 \mathrm{~mL})$. $\mathrm{Et}_{3} \mathrm{~N}(10 \mathrm{mmol})$ was taken to the vigorously stirred solution via a syringe. The reaction mixture was stirred at room temperature for $12 \mathrm{~h}$ and quenched with saturated $\mathrm{NaHCO}_{3} \cdot \mathrm{H}_{2} \mathrm{O}$ was added to the mixture and extracted with DCM. Combined organic phase was washed with saturated brine and dried over $\mathrm{Na}_{2} \mathrm{SO}_{4}$, and then filtered, the solvent was removed in a rotary evaporator. The crude product was purified by silica gel column with petroleum ether/ethyl acetate eluent gave the desired product $\mathbf{1} \mathbf{w}$ or $\mathbf{1 x}$.

\section{Nickel-promoted $\mathrm{C}-\mathrm{H}$ amination to form primary anilines}

\subsection{Further optimization of the reaction conditions}

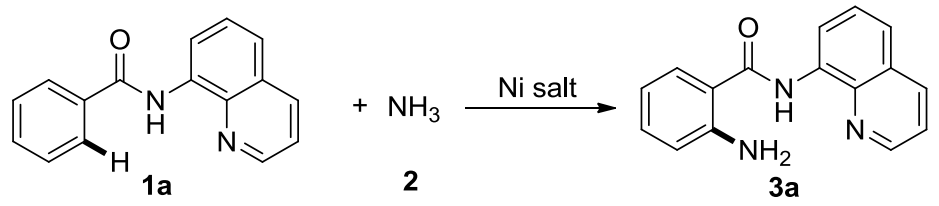

\begin{tabular}{lllllll}
\hline Entry & $\mathrm{NiCl}_{2}$ (equiv) & AgTFA (equiv) & TBAA (equiv) & Solvent & Temp $\left({ }^{\circ} \mathrm{C}\right)$ & Yield $(\%)^{a, b}$ \\
\hline 1 & 2 & 2 & 2 & DMSO & 140 & 68 \\
2 & 1 & 2 & 2 & DMSO & 140 & 38 \\
3 & 0 & 2 & 2 & DMSO & 140 & 0 \\
4 & 2 & 1 & 2 & DMSO & 140 & 65 \\
5 & 2 & 0 & 2 & DMSO & 140 & 63 \\
\hline
\end{tabular}




\begin{tabular}{|c|c|c|c|c|c|c|}
\hline 6 & 2 & 0 & 1 & DMSO & 140 & 50 \\
\hline 7 & 2 & 0 & 0 & DMSO & 140 & 26 \\
\hline 7 & 2 & 0 & 2 & NMP & 140 & 65 \\
\hline 8 & 2 & 0 & 2 & NMP & 130 & 63 \\
\hline $9^{c}$ & 2 & 0 & 2 & NMP & 120 & 66 \\
\hline $10^{d}$ & 2 & 0 & 2 & NMP & 110 & 47 \\
\hline $11^{e}$ & 0.5 & 0 & 2 & NMP & 120 & 21 \\
\hline $12^{f}$ & 0.5 & 0 & 2 & NMP & 120 & $<10$ \\
\hline $13^{g}$ & 0.5 & 0 & 2 & NMP & 120 & $<10$ \\
\hline $14^{h}$ & 0.5 & 0 & 2 & NMP & 120 & trace \\
\hline $15^{i}$ & 0.1 & 2 & 2 & NMP & 120 & $<10$ \\
\hline $16^{j}$ & 0.1 & 2 & 2 & NMP & 120 & $<10$ \\
\hline $17^{k}$ & 0.1 & 2 & 2 & NMP & 120 & $<10$ \\
\hline $18^{l}$ & 2 & 0 & 2 & NMP & 120 & 18 \\
\hline
\end{tabular}

${ }^{a}$ Reaction conditions: amide $1 \mathrm{a}(0.15 \mathrm{mmol}), \mathrm{NiCl}_{2}$ (x mmol), $\mathrm{K}_{2} \mathrm{CO}_{3}(0.3 \mathrm{mmol})$, TBAA (x mmol), Solvent (5.0 $\mathrm{mL}$ ), reaction run in a sealed tube under $\mathrm{NH}_{3}$ ( $1 \mathrm{~atm}$, closed) for $2 \mathrm{~h} .{ }^{b}$ Isolated yield. ${ }^{c} 12$ hours. ${ }^{d} 24$ hours. ${ }^{e}$ Add 0.3 mmol NMO as oxidant. ${ }^{f}$ Add 0.3 mmol $\mathrm{Ag}_{2} \mathrm{O}$ as oxidant. ${ }^{g}$ Add $0.3 \mathrm{mmol} \mathrm{MnO}_{2}$ as oxidant. ${ }^{h}$ Add $0.3 \mathrm{mmol} \mathrm{K}_{2} \mathrm{~S}_{2} \mathrm{O}_{8}$ as oxidant. ${ }^{i}$ Add $0.03 \mathrm{mmol} \mathrm{PPh}_{3}$ as ligand. ${ }^{j}$ Add $0.015 \mathrm{mmol}$ dppe as ligand. ${ }^{k}$ Add $0.015 \mathrm{mmol}$ 1,10-phen as ligand. ' $\mathrm{using}$ aqueous ammonia instead of gaseous ammonia and under $\mathrm{N}_{2}$ atmosphere.

\subsection{General procedure for nickel-promoted C-H amination to form}

\section{primary anilines}

Aromatic amide 1 (0.15 mmol), $\mathrm{NiCl}_{2}(39 \mathrm{mg}, 0.3 \mathrm{mmol}), \mathrm{K}_{2} \mathrm{CO}_{3}(42 \mathrm{mg}, 0.3 \mathrm{mmol})$, TBAA (90 mg, $0.3 \mathrm{mmol})$ and NMP (5.0 mL) were added to a $35 \mathrm{~mL}$ Schlenk flask equipped with a high-vacuum PTFE valve-to-glass seal. After air-evacuation and being refilled with $\mathrm{NH}_{3}(1 \mathrm{~atm})$ three times, ammonia gas was injected for 10 minutes via a balloon. Then the flask was sealed under $\mathrm{NH}_{3}(1 \mathrm{~atm})$ and stirred at $120{ }^{\circ} \mathrm{C}$ for $12 \mathrm{~h}$. After the reaction was quenched by addition of brine, the mixture was extracted with dichloromethane, and the combined organic layer was dried over sodium sulfate. Concentration in vacuo followed by silica gel column purification with petroleum ether /ethyl acetate/triethylamine eluent gave the desired product 3. (The procedure of 
reaction condition $\mathrm{B}$ are similar to those of reaction condition $\mathrm{A})$.

\subsection{Procedure for $1 \mathrm{mmol}$ scale reaction with $1 \mathrm{a}$}

A dried round bottom flask charged with benzamide 1a (248 mg, $1.0 \mathrm{mmol}), \mathrm{NiCl}_{2}$ (259.2 mg, $2.0 \mathrm{mmol}), \mathrm{K}_{2} \mathrm{CO}_{3}(276.4 \mathrm{mg}, 2.0 \mathrm{mmol})$, TBAA (603 mg, $\left.2.0 \mathrm{mmol}\right)$, AgTFA (441.8 mg, $2.0 \mathrm{mmol})$, was evacuated and purged with argon. DMSO (30 mL) was added by syringe under argon. After air-evacuation and being refilled with $\mathrm{NH}_{3}$ (1 atm) three times, ammonia gas was injected for 10 minutes via a balloon. Then the flask was sealed under $\mathrm{NH}_{3}$ and stirred at $140{ }^{\circ} \mathrm{C}$ for $2 \mathrm{~h}$. After the reaction was quenched by addition of brine, the mixture was extracted with dichloromethane, and the combined organic layer was dried over sodium sulfate. Concentration in vacuo followed by silica gel column purification with petroleum ether /ethyl acetate/triethylamine eluent (80:10:1) gave the desired product 3a in 65\% yield (171 $\mathrm{mg})$.

\subsection{Deuterium-labeling experiments.}

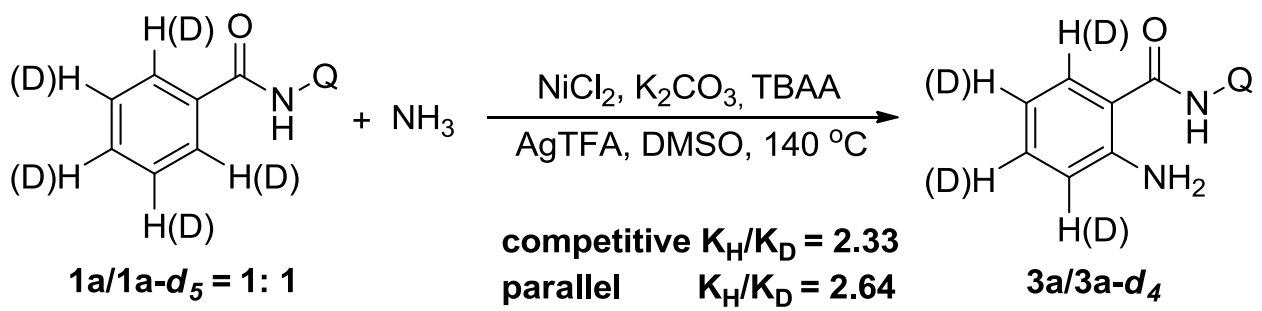

Intermolecular competition KIE Following general procedure: 1a (37 mg, 0.15 mmol), 1a-d $\boldsymbol{d}_{5}(38 \mathrm{mg}, 0.15 \mathrm{mmol}), \mathrm{NiCl}_{2}(78 \mathrm{mg}, 0.6 \mathrm{mmol}), \mathrm{K}_{2} \mathrm{CO}_{3}(83 \mathrm{mg}, 0.6$ mmol), tetrabutylammonium acetate $(181 \mathrm{mg}, 0.6 \mathrm{mmol}), \operatorname{AgTFA}(133 \mathrm{mg}, 0.6 \mathrm{mmol})$ and DMSO (10.0 mL) were added to a $75 \mathrm{~mL}$ Schlenk flask equipped with a high-vacuum PTFE valve-to-glass seal. The flask was injected with ammonia gas for 10 minutes. Then the flask was sealed under $\mathrm{NH}_{3}$ and stirred at $140{ }^{\circ} \mathrm{C}$ for $8 \mathrm{~min}$. The product was separated by column chromatography to give the desired product in $15 \%$ yield.

${ }^{1} \mathrm{H}$ NMR $\left(400 \mathrm{MHz}, \mathrm{CDCl}_{3}\right): \delta 10.64(\mathrm{~s}, 1.00 \mathrm{H}), 8.89-8.83(\mathrm{~m}, 2.00 \mathrm{H}), 8.19(\mathrm{~d}, J=$ $8.2 \mathrm{~Hz}, 1.00 \mathrm{H}), 7.79(\mathrm{~d}, J=7.9 \mathrm{~Hz}, 0.69 \mathrm{H}), 7.62-7.51(\mathrm{~m}, 2.00 \mathrm{H}), 7.48(\mathrm{dd}, J=6.1$, 
$3.9 \mathrm{~Hz}, 1.00 \mathrm{H}), 7.29(\mathrm{t}, J=7.6 \mathrm{~Hz}, 0.71 \mathrm{H}), 6.80(\mathrm{t}, J=7.5 \mathrm{~Hz}, 0.70 \mathrm{H}), 6.75(\mathrm{~d}, J=$ $8.2 \mathrm{~Hz}, 0.70 \mathrm{H}), 5.73(\mathrm{~s}, 2.00 \mathrm{H})$. The KIE value was calculated as $\mathrm{k}_{\mathrm{H}} / \mathrm{k}_{\mathrm{D}}=2.33$.

Intermolecular parallel KIE Following general procedure: $1 \mathrm{a}(37 \mathrm{mg}, 0.15 \mathrm{mmol})$ or $\mathbf{1 a}-\boldsymbol{d}_{5}(38 \mathrm{mg}, 0.15 \mathrm{mmol}), \mathrm{NiCl}_{2}(39 \mathrm{mg}, 0.3 \mathrm{mmol}), \mathrm{K}_{2} \mathrm{CO}_{3}(42 \mathrm{mg}, 0.15 \mathrm{mmol})$, tetrabutylammonium acetate (90 mg, $0.3 \mathrm{mmol})$, AgTFA (66 mg, $0.3 \mathrm{mmol})$ and DMSO (5.0 mL) were added to a $75 \mathrm{~mL}$ Schlenk flask equipped with a high-vacuum PTFE valve-to-glass seal. Then the flask was sealed under $\mathrm{NH}_{3}$ and stirred at $140{ }^{\circ} \mathrm{C}$ for $8 \mathrm{~min}$. The product was separated by column chromatography to give the desired product in $12 \%$ yield.

${ }^{1} \mathrm{H}$ NMR $\left(400 \mathrm{MHz}, \mathrm{CDCl}_{3}\right): \delta 10.57(\mathrm{~s}, 1.00 \mathrm{H}), 8.85-8.75(\mathrm{~m}, 2.00 \mathrm{H}), 8.11(\mathrm{~d}, J=$ $8.3 \mathrm{~Hz}, 1.00 \mathrm{H}), 7.71(\mathrm{~d}, J=7.8 \mathrm{~Hz}, 0.73 \mathrm{H}), 7.55-7.44(\mathrm{~m}, 2.00 \mathrm{H}), 7.48(\mathrm{dd}, J=8.2$, $4.2 \mathrm{~Hz}, 1.00 \mathrm{H}), 7.22(\mathrm{t}, J=7.7 \mathrm{~Hz}, 0.72 \mathrm{H}), 6.73(\mathrm{t}, J=7.4 \mathrm{~Hz}, 0.73 \mathrm{H}), 6.68(\mathrm{~d}, J=$ $8.1 \mathrm{~Hz}, 0.72 \mathrm{H}), 5.66(\mathrm{~s}, 2.00 \mathrm{H})$. The KIE value was calculated as $\mathrm{k}_{\mathrm{H}} / \mathrm{k}_{\mathrm{D}}=2.64$.

${ }^{1} \mathrm{H}$ NMR Spectrum of Intermolecular competition
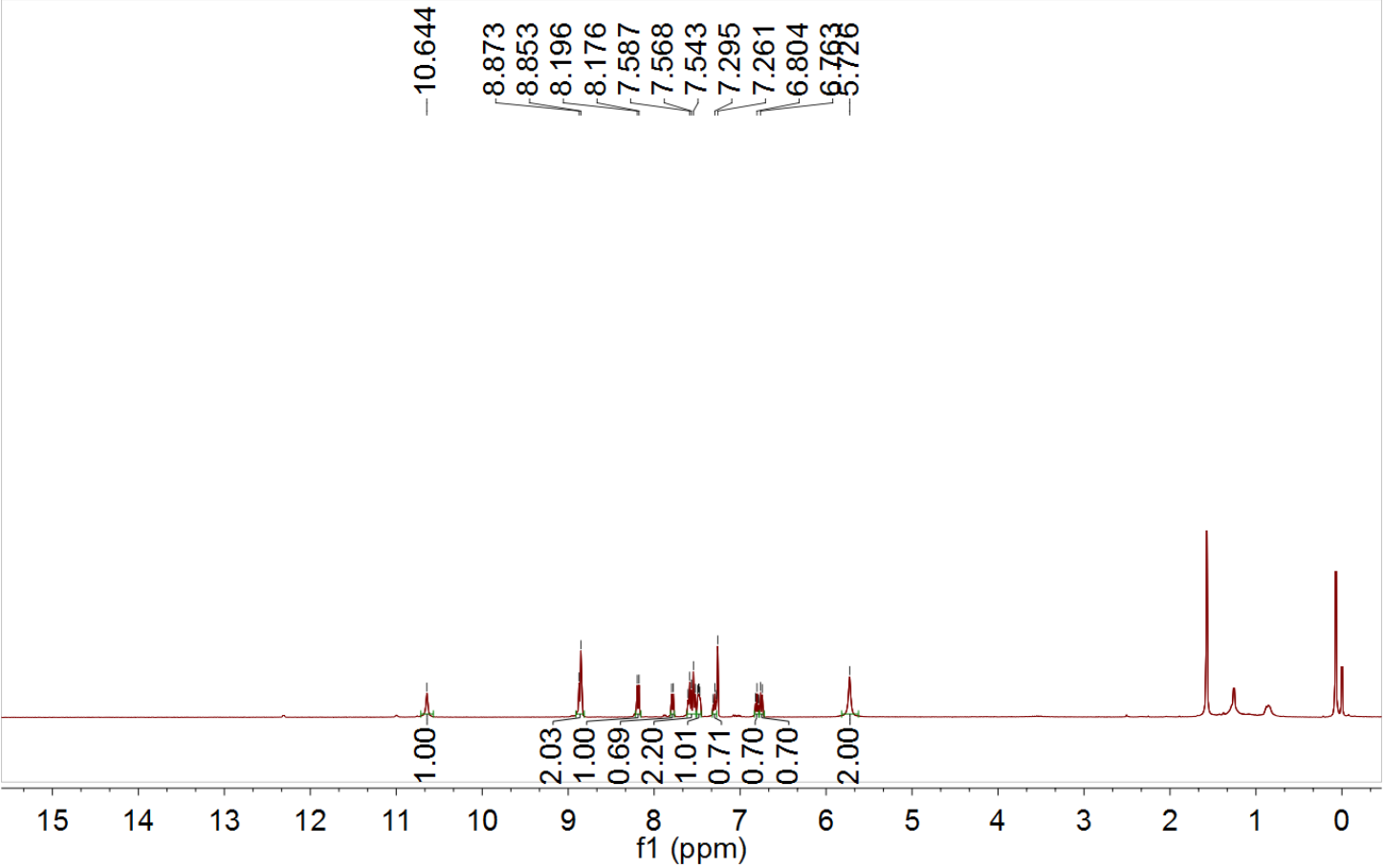
${ }^{1} \mathrm{H}$ NMR Spectrum of Intermolecular parallel

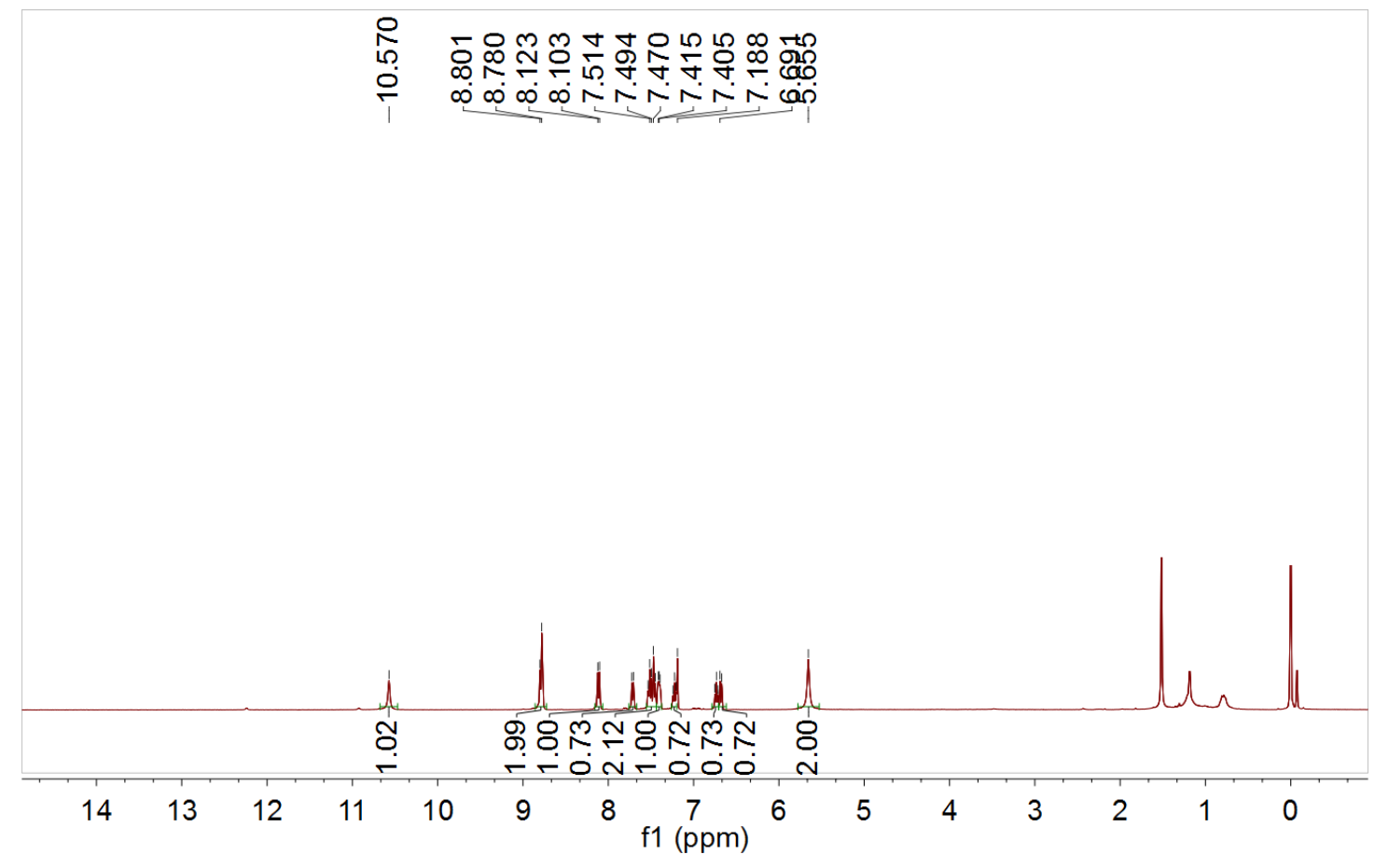

${ }^{1} \mathrm{H}$ NMR Spectrum of recovered amide (1a-d $)$ : We didn't observed the H/D exchange in the recovered amide in the deuterium labelling experiments.

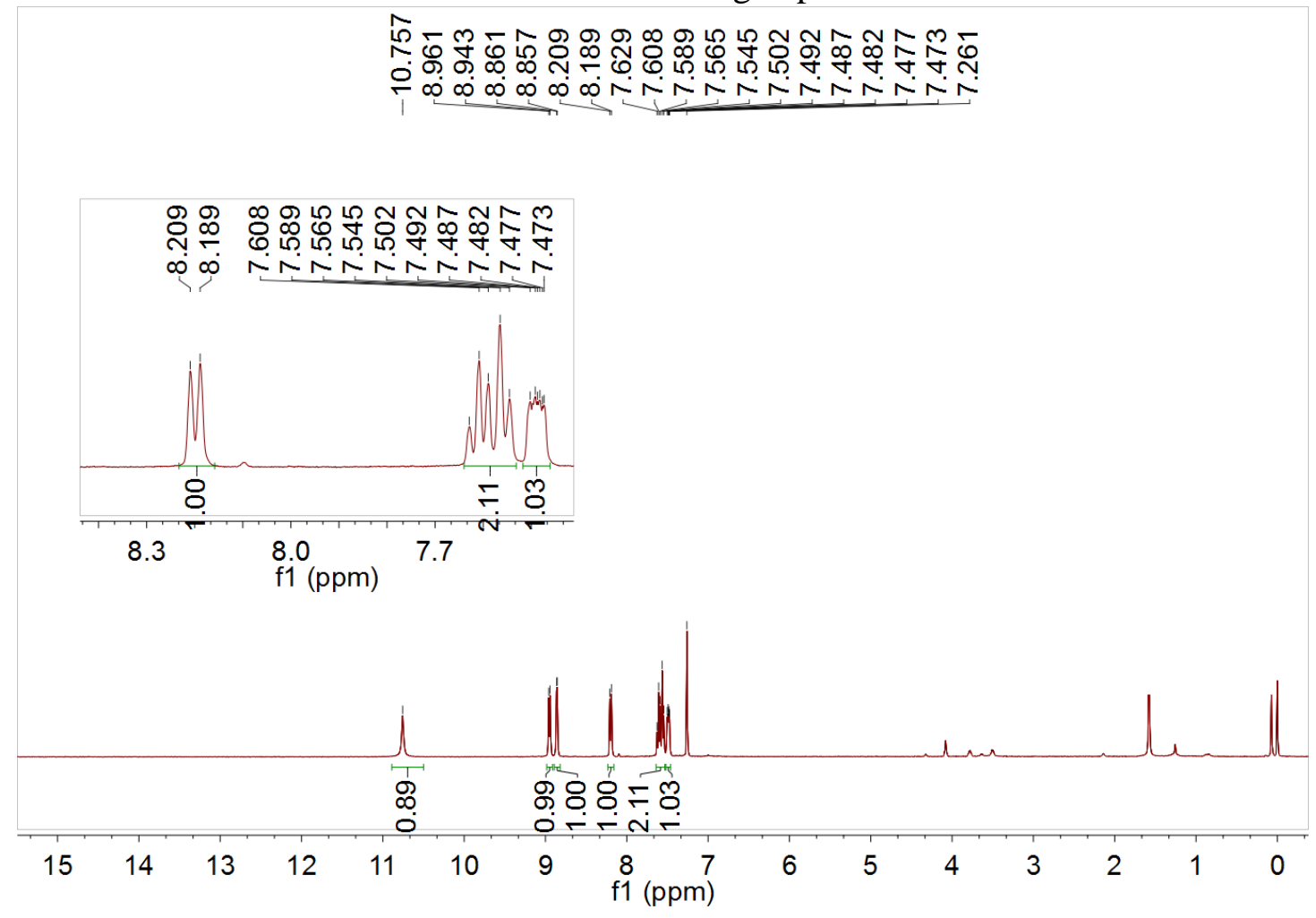




\section{Characterization data of products.}

\section{4-(pyridin-2-yl)- $N$-(quinolin-8-yl)benzamide (1w):}<smiles>O=C(Nc1cccc2cccnc12)c1ccc(-c2ccccn2)cc1</smiles>

Following the general procedure the title compound was isolated by flash chromatography (eluent: petrol ether/ethyl acetate $=2 / 1$ ) as a white solid in $83 \%$ yield (1.349 g), mp 152-153 ${ }^{\circ} \mathrm{C} .{ }^{1} \mathrm{H}$ NMR (400 MHz, $\left.\mathrm{CDCl}_{3}\right): \delta 10.79(\mathrm{~s}, 1 \mathrm{H}), 8.95(\mathrm{~d}$, $J=7.4 \mathrm{~Hz}, 1 \mathrm{H}), 8.84(\mathrm{~d}, J=1.9 \mathrm{~Hz}, 1 \mathrm{H}), 8.72(\mathrm{~d}, J=3.1 \mathrm{~Hz}, 1 \mathrm{H}), 8.25-8.12(\mathrm{~m}, 5 \mathrm{H})$, 7.83-7.74 (m, 2H), 7.61-7.50 (m, 2H), 748-7.42 (m, 1H), 7.29-7.23 (m, $1 \mathrm{H}) ;{ }^{13} \mathrm{C}$ NMR (100 MHz, $\left.\mathrm{CDCl}_{3}\right): \delta 165.0,156.1,149.8,148.3,142.5,138.7,136.8,136.3$, 135.2, 134.5, 127.9, 127.7, 127.4, 127.2, 122.7, 121.7, 121.6, 120.8, 116.5; HRMS (ESI, m/z): calcd for $\mathrm{C}_{21} \mathrm{H}_{15} \mathrm{~N}_{3} \mathrm{O}[\mathrm{M}+\mathrm{H}]^{+}$: 326.1288; Found: 326.1302 .

\section{4-(1H-pyrazol-1-yl)- $N$-(quinolin-8-yl)benzamide (1x):}<smiles>O=C(Nc1cccc2cccnc12)c1ccc(-n2cccn2)cc1</smiles>

Following the general procedure the title compound was isolated by flash chromatography (eluent: petrol ether/ethyl acetate $=2 / 1$ ) as a white solid in $62 \%$ yield $(0.973 \mathrm{~g}), \mathrm{mp} 183-184{ }^{\circ} \mathrm{C} .{ }^{1} \mathrm{H}$ NMR $\left(400 \mathrm{MHz}, \mathrm{CDCl}_{3}\right): \delta 10.74(\mathrm{~s}, 1 \mathrm{H}), 8.92(\mathrm{~d}$, $J=6.9 \mathrm{~Hz}, 1 \mathrm{H}), 8.84(\mathrm{~d}, J=2.6 \mathrm{~Hz}, 1 \mathrm{H}), 8.24-8.10(\mathrm{~m}, 3 \mathrm{H}), 8.01(\mathrm{~d}, J=1.9 \mathrm{~Hz}, 1 \mathrm{H})$, $7.87(\mathrm{~d}, J=8.6,2 \mathrm{H}), 7.77(\mathrm{~s}, 1 \mathrm{H}), 7.62-7.50(\mathrm{~m}, 2 \mathrm{H}), 7.46(\mathrm{dd}, J=8.2,4.2 \mathrm{~Hz}, 1 \mathrm{H})$, $6.51(\mathrm{~s}, 1 \mathrm{H}) ;{ }^{13} \mathrm{C} \mathrm{NMR}\left(100 \mathrm{MHz}, \mathrm{CDCl}_{3}\right): \delta 164.3,148.3,142.5,141.8,138.7,136.3$, 134.4, 132.6, 128.7, 127.9, 127.4, 126.8, 121.7, 121.7, 118.7, 116.5, 108.3; HRMS (ESI, m/z): calcd for $\mathrm{C}_{19} \mathrm{H}_{14} \mathrm{~N}_{4} \mathrm{O}[\mathrm{M}+\mathrm{H}]^{+}: 315.1240$; Found: 315.1255 .

\section{2-amino- $N$-(2-(pyridin-2-yl)propan-2-yl)benzamide (3ac):}<smiles>CC(C)(NC(=O)c1ccccc1N)c1ccccn1</smiles>

3ac

Following the general procedure the title compound was isolated by flash chromatography (eluent: petrol ether/ethyl acetate/triethylamine $=80 / 10 / 1$ ) as a semisolid in $9 \%$ yield $(3.4 \mathrm{mg}),{ }^{1} \mathrm{H} \mathrm{NMR}\left(400 \mathrm{MHz}, \mathrm{CDCl}_{3}\right): \delta 8.54(\mathrm{~d}, J=3.9 \mathrm{~Hz}$, $1 \mathrm{H}), 8.46(\mathrm{~s}, 1 \mathrm{H}), 7.71(\mathrm{t}, J=7.6 \mathrm{~Hz}, 1 \mathrm{H}), 7.53(\mathrm{~d}, J=7.8 \mathrm{~Hz}, 1 \mathrm{H}), 7.44(\mathrm{~d}, J=8.0$ $\mathrm{Hz}, 1 \mathrm{H}), 7.19(\mathrm{t}, J=6.8,2 \mathrm{H}), 6.73-6.64(\mathrm{~m}, 2 \mathrm{H}), 5.55(\mathrm{~s}, 2 \mathrm{H}), 1.85(\mathrm{~s}, 6 \mathrm{H}) ;{ }^{13} \mathrm{C} \mathrm{NMR}$ $\left(100 \mathrm{MHz}, \mathrm{CDCl}_{3}\right): \delta 168.6,164.7,148.6,147.6,137.0,131.7,127.6,121.8,119.4$, 
117.6, 117.1, 116.5, 56.6, 27.6; HRMS (ESI, m/z): calcd for $\mathrm{C}_{15} \mathrm{H}_{17} \mathrm{~N}_{3} \mathrm{O}[\mathrm{M}+\mathrm{H}]^{+}$: 256.1444; Found: 256.1450.

\section{2-amino- $N$-(quinolin-8-yl)benzamide (3a):}<smiles>Nc1ccccc1C(=O)Nc1cccc2cccnc12</smiles>

3a

Following the general procedure the title compound was isolated by flash chromatography (eluent: petrol ether/ethyl acetate/triethylamine $=80 / 10 / 1$ ) as a white solid in $66 \%(68 \%)$ yield $(26.7 \mathrm{mg})$. This compound is known ${ }^{4} .{ }^{1} \mathrm{H}$ NMR $(400 \mathrm{MHz}$, $\left.\mathrm{CDCl}_{3}\right): \delta 10.53(\mathrm{~s}, 1 \mathrm{H}), 8.78-8.72(\mathrm{~m}, 2 \mathrm{H}), 8.05(\mathrm{~d}, J=8.2 \mathrm{~Hz}, 1 \mathrm{H}), 7.68(\mathrm{~d}, J=7.9$ $\mathrm{Hz}, 1 \mathrm{H}), 7.51-7.38(\mathrm{~m}, 2 \mathrm{H}), 7.34(\mathrm{dd}, J=8.1,4.2 \mathrm{~Hz}, 1 \mathrm{H}), 7.19(\mathrm{t}, J=7.6,1 \mathrm{H}), 6.69$ $(\mathrm{t}, J=7.5 \mathrm{~Hz}, 1 \mathrm{H}), 6.65(\mathrm{~d}, J=8.2 \mathrm{~Hz}, 1 \mathrm{H}), 5.65(\mathrm{~s}, 2 \mathrm{H}) ;{ }^{13} \mathrm{C}$ NMR $(100 \mathrm{MHz}$, $\left.\mathrm{CDCl}_{3}\right): \delta 167.6,149.4,148.2,138.8,136.3,134.7,132.7,128.0,127.6,127.3,121.6$, $121.4,117.5,116.7,116.2$.

\section{2-amino-4-methyl- $N$-(quinolin-8-yl)benzamide (3b):}<smiles>Cc1ccc(C(=O)Nc2cccc3cccnc23)c(N)c1</smiles>

$3 \mathbf{b}$

Following the general procedure the title compound was isolated by flash chromatography (eluent: petrol ether/ethyl acetate/triethylamine $=80 / 10 / 1$ ) as a white solid in $45 \%$ (51\%) yield (21.2 mg). This compound is known ${ }^{4} .{ }^{1} \mathrm{H}$ NMR (400 MHz, $\left.\mathrm{CDCl}_{3}\right): \delta 10.61(\mathrm{~s}, 1 \mathrm{H}), 8.89-8.80(\mathrm{~m}, 2 \mathrm{H}), 8.15(\mathrm{~d}, J=8.2 \mathrm{~Hz}, 1 \mathrm{H}), 7.68(\mathrm{~d}, J=8.1$ $\mathrm{Hz}, 1 \mathrm{H}), 7.57(\mathrm{t}, J=7.9 \mathrm{~Hz}, 1 \mathrm{H}), 7.50(\mathrm{~d}, J=8.2 \mathrm{~Hz}, 1 \mathrm{H}), 7.44(\mathrm{dd}, J=8.2,4.2 \mathrm{~Hz}$, $1 \mathrm{H}), 6.61(\mathrm{~d}, J=8.1 \mathrm{~Hz}, 1 \mathrm{H}), 6.55(\mathrm{~s}, 1 \mathrm{H}), 5.75(\mathrm{~s}, 2 \mathrm{H}), 2.31(\mathrm{~s}, 3 \mathrm{H}) ;{ }^{13} \mathrm{C} \mathrm{NMR}(100$ $\mathrm{MHz}, \mathrm{CDCl}_{3}$ ): $\delta 167.6,149.6,148.1,143.3,138.8,136.2,134.8,128.0,127.6,127.3$, $121.5,121.2,118.0,117.7,116.1,113.5,21.4$.

2-amino-4-methoxy- $N$-(quinolin-8-yl)benzamide (3c):<smiles>COc1ccc(C(=O)Nc2cccc3cccnc23)c(N)c1</smiles>

Following the general procedure the title compound was isolated by flash chromatography (eluent: petrol ether/ethyl acetate/triethylamine $=80 / 10 / 1$ ) as a white solid in $51 \%$ (62\%) yield $(27.3 \mathrm{mg})$. This compound is known ${ }^{4} .{ }^{1} \mathrm{H}$ NMR (400 MHz, 
$\left.\mathrm{CDCl}_{3}\right): \delta 10.55(\mathrm{~s}, 1 \mathrm{H}), 8.83(\mathrm{~d}, J=5.5 \mathrm{~Hz}, 2 \mathrm{H}), 8.16(\mathrm{~d}, J=8.2 \mathrm{~Hz}, 1 \mathrm{H}), 7.73(\mathrm{~d}, J$ $=8.7 \mathrm{~Hz}, 1 \mathrm{H}), 7.60-7.41(\mathrm{~m}, 3 \mathrm{H}), 6.37(\mathrm{~d}, J=8.7 \mathrm{~Hz}, 1 \mathrm{H}), 6.20(\mathrm{~s}, 1 \mathrm{H}), 5.93(\mathrm{~s}, 2 \mathrm{H})$, $3.82(\mathrm{~s}, 3 \mathrm{H}) ;{ }^{13} \mathrm{C}$ NMR $\left(100 \mathrm{MHz}, \mathrm{CDCl}_{3}\right): \delta 167.4,163.2,151.7,148.1,138.8,136.3$, 135.0, 129.4, 128.0, 127.4, 121.6, 121.0, 116.0, 109.3, 104.4, 100.6, 55.2.

2-amino- $N$-(quinolin-8-yl)-4-vinylbenzamide (3d):<smiles>C=Cc1ccc(C(=O)Nc2cccc3cccnc23)c(N)c1</smiles>

3d

Following the general procedure the title compound was isolated by flash chromatography (eluent: petrol ether/ethyl acetate/triethylamine $=80 / 10 / 1$ ) as a white solid in 50\% (53\%) yield (23.0 mg). This compound is known ${ }^{4} .{ }^{1} \mathrm{H}$ NMR (400 MHz, $\left.\mathrm{CDCl}_{3}\right): \delta 10.64(\mathrm{~s}, 1 \mathrm{H}), 8.85(\mathrm{~d}, J=7.2 \mathrm{~Hz}, 2 \mathrm{H}), 8.19(\mathrm{~d}, J=8.2 \mathrm{~Hz}, 1 \mathrm{H}), 7.76(\mathrm{~d}, J$ $=8.0 \mathrm{~Hz}, 1 \mathrm{H}), 7.62-7.50(\mathrm{~m}, 2 \mathrm{H}), 7.47(\mathrm{dd}, J=6.4,3.9 \mathrm{~Hz}, 1 \mathrm{H}), 6.89(\mathrm{~d}, J=8.1 \mathrm{~Hz}$, $1 \mathrm{H}), 6.74(\mathrm{~s}, 1 \mathrm{H}), 6.66(\mathrm{dd}, J=17.5,10.8 \mathrm{~Hz}, 1 \mathrm{H}), 5.82(\mathrm{~d}, J=17.6 \mathrm{~Hz}, 1 \mathrm{H}), 5.34(\mathrm{~d}$, $J=10.8 \mathrm{~Hz}, 1 \mathrm{H})$; Due to $\mathrm{H} / \mathrm{D}$ exchange of $\mathrm{NH}_{2}$ with $\mathrm{CDCl}_{3}, \mathrm{NH}_{2}$ disappear. ${ }^{13} \mathrm{C}$ NMR (100 MHz, $\left.\mathrm{CDCl}_{3}\right): \delta 167.4,149.7,148.2,141.7,138.8,136.3,136.2,134.8$, $128.0,128.0,127.4,121.6,121.3,116.2,115.9,115.4,115.3,114.6$.

\section{2-amino-4-phenyl- $N$-(quinolin-8-yl)benzamide (3e):}<smiles>Nc1cc(-c2ccccc2)ccc1C(=O)Nc1cccc2cccnc12</smiles>

$3 e$

Following the general procedure the title compound was isolated by flash chromatography (eluent: petrol ether/ethyl acetate/triethylamine $=80 / 10 / 1$ ) as a white solid in $46 \%(66 \%)$ yield (33.6 mg). This compound is known ${ }^{4} .{ }^{1} \mathrm{H}$ NMR (400 MHz, $\left.\mathrm{CDCl}_{3}\right): \delta 10.70(\mathrm{~s}, 1 \mathrm{H}), 8.93-8.84(\mathrm{~m}, 2 \mathrm{H}), 8.18(\mathrm{~d}, J=8.2 \mathrm{~Hz}, 1 \mathrm{H}), 7.86(\mathrm{~d}, J=8.2$ $\mathrm{Hz}, 1 \mathrm{H}), 7.66-7.36(\mathrm{~m}, 8 \mathrm{H}), 7.04(\mathrm{~d}, J=8.1 \mathrm{~Hz}, 1 \mathrm{H}), 6.96(\mathrm{~s}, 1 \mathrm{H}), 5.87(\mathrm{~s}, 2 \mathrm{H}) ;{ }^{13} \mathrm{C}$ NMR (100 MHz, $\left.\mathrm{CDCl}_{3}\right): \delta 167.5,149.8,148.2,145.5,140.3,138.8,136.3,134.8$, $128.8,128.2,128.0,127.9,127.4,127.1,121.6,121.4,116.2,115.9,115.8,115.0$.

2-amino-4-cyano- $N$-(quinolin-8-yl)benzamide (3f):<smiles>N#Cc1ccc(C(=O)Nc2cccc3cccnc23)c(N)c1</smiles>

$3 \mathbf{f}$ 
Following the general procedure the title compound was isolated by flash chromatography (eluent: petrol ether/ethyl acetate/triethylamine $=80 / 10 / 1$ ) as a yellow solid in 63\% (75\%) yield (34.9 mg), mp 165-166 ${ }^{\circ} \mathrm{C} .{ }^{1} \mathrm{H}$ NMR (400 MHz, $\left.\mathrm{CDCl}_{3}\right): \delta 10.64(\mathrm{~s}, 1 \mathrm{H}), 8.90-8.75(\mathrm{~m}, 2 \mathrm{H}), 8.19(\mathrm{~d}, J=8.2 \mathrm{~Hz}, 1 \mathrm{H}), 7.80(\mathrm{~d}, J=8.0$ $\mathrm{Hz}, 1 \mathrm{H}), 7.62-7.50(\mathrm{~m}, 2 \mathrm{H}), 7.49$ (dd, $J=7.2,3.5 \mathrm{~Hz}, 1 \mathrm{H}), 7.04-6.96(\mathrm{~m}, 2 \mathrm{H}), 5.91$ (s, $2 \mathrm{H}) ;{ }^{13} \mathrm{C} \mathrm{NMR}\left(100 \mathrm{MHz}, \mathrm{CDCl}_{3}\right): \delta 166.1,149.2,148.4,138.7,136.4,134.1,128.4$, 128.0, 127.3, 122.1, 121.8, 120.4, 119.5, 119.3, 118.3, 116.5, 115.7; HRMS (ESI, m/z): calcd for $\mathrm{C}_{17} \mathrm{H}_{12} \mathrm{~N}_{4} \mathrm{O}[\mathrm{M}+\mathrm{Na}]^{+}:$311.0903; Found: 311.0917 .

methyl 3-amino-4-(quinolin-8-ylcarbamoyl)benzoate (3g):<smiles>CC(=O)c1ccc(C(=O)Nc2cccc3cccnc23)c(N)c1</smiles>

$3 g$

Following the general procedure the title compound was isolated by flash chromatography (eluent: petrol ether/ethyl acetate/triethylamine $=80 / 10 / 1$ ) as a yellow solid in $68 \%$ (73\%) yield $\left(35.2 \mathrm{mg}\right.$ ). This compound is known ${ }^{4} .{ }^{1} \mathrm{H}$ NMR (400 $\left.\mathrm{MHz}, \mathrm{CDCl}_{3}\right): \delta 10.65(\mathrm{~s}, 1 \mathrm{H}), 8.84(\mathrm{~d}, J=3.7 \mathrm{~Hz}, 2 \mathrm{H}), 8.17(\mathrm{~d}, J=8.1 \mathrm{~Hz}, 1 \mathrm{H}), 7.80$ $(\mathrm{d}, J=8.0 \mathrm{~Hz}, 1 \mathrm{H}), 7.60-7.44(\mathrm{~m}, 3 \mathrm{H}), 7.41(\mathrm{~d}, J=8.5 \mathrm{~Hz}, 2 \mathrm{H}), 5.81(\mathrm{~s}, 2 \mathrm{H}), 3.92$ (s, $3 \mathrm{H}) ;{ }^{13} \mathrm{C}$ NMR $\left(100 \mathrm{MHz}, \mathrm{CDCl}_{3}\right): \delta 166.8,166.5,149.1,148.3,138.7,136.4,134.4$, $133.6,128.0,127.8,127.3,121.8,121.7,119.5,118.5,117.3,116.4,52.3$.

2-amino- $N$-(quinolin-8-yl)-4-(trifluoromethoxy)benzamide (3h):<smiles>Nc1cc(OC(F)(F)F)ccc1C(=O)Nc1cccc2cccnc12</smiles>

$3 h$

Following the general procedure the title compound was isolated by flash chromatography (eluent: petrol ether/ethyl acetate/triethylamine $=80 / 10 / 1$ ) as a white solid in $40(54 \%)$ yield $(28.1 \mathrm{mg})$. This compound is known ${ }^{4}{ }^{1} \mathrm{H}$ NMR $(400 \mathrm{MHz}$, $\left.\mathrm{CDCl}_{3}\right): \delta 10.59(\mathrm{~s}, 1 \mathrm{H}), 8.83(\mathrm{~d}, J=7.4 \mathrm{~Hz}, 2 \mathrm{H}), 8.19(\mathrm{~d}, J=8.1 \mathrm{~Hz}, 1 \mathrm{H}), 7.79(\mathrm{~d}, J$ $=8.7 \mathrm{~Hz}, 1 \mathrm{H}), 7.63-7.51(\mathrm{~m}, 2 \mathrm{H}), 7.48(\mathrm{dd}, J=8.2,4.2 \mathrm{~Hz}, 1 \mathrm{H}), 6.63(\mathrm{~d}, J=8.6 \mathrm{~Hz}$, $1 \mathrm{H}), 6.56(\mathrm{~s}, 1 \mathrm{H}), 5.93(\mathrm{~s}, 2 \mathrm{H}) ;{ }^{13} \mathrm{C} \mathrm{NMR}\left(100 \mathrm{MHz}, \mathrm{CDCl}_{3}\right): \delta 166.7,152.4\left(\mathrm{q}, J_{\mathrm{C}-\mathrm{F}}=\right.$ $2.0 \mathrm{~Hz}), 151.0,148.3,138.8,136.4,134.5,129.5$, 128.0, 127.4, 121.7, 121.6, 116.3, 114.5, 108.5, 108.2; ${ }^{19} \mathrm{~F}$ NMR (377 MHz, $\left.\mathrm{CDCl}_{3}\right) \delta-57.3$.

2-amino- $N$-(quinolin-8-yl)-4-(trifluoromethoxy)benzamide (3i): 
<smiles>Nc1cc(C(F)(F)F)ccc1C(=O)Nc1cccc2cccnc12</smiles>

3i

Following the general procedure the title compound was isolated by flash chromatography (eluent: petrol ether/ethyl acetate/triethylamine $=80 / 10 / 1$ ) as a white solid in 74\% (81\%) yield (40.2 mg), mp 122-123 ${ }^{\circ} \mathrm{C} .{ }^{1} \mathrm{H}$ NMR (400 MHz, $\left.\mathrm{CDCl}_{3}\right): \delta$ $10.65(\mathrm{~s}, 1 \mathrm{H}), 8.83(\mathrm{~d}, J=4.3 \mathrm{~Hz}, 2 \mathrm{H}), 8.18(\mathrm{~d}, J=8.2 \mathrm{~Hz}, 1 \mathrm{H}), 7.85(\mathrm{~d}, J=8.0 \mathrm{~Hz}$, 1H), 7.61-7.53 (m, 2H), 7.51-7.44 (m, 1H), 7.07-6.93 (m, 2H), $5.90(\mathrm{~s}, 2 \mathrm{H}) ;{ }^{13} \mathrm{C} \mathrm{NMR}$ $\left(100 \mathrm{MHz}, \mathrm{CDCl}_{3}\right): \delta 166.6,149.2,148.4,138.7,136.4,134.3,134.2$ (q, $J_{\mathrm{C}-\mathrm{F}}=32.1$ $\mathrm{Hz}), 128.4,128.0,127.3,123.6\left(\mathrm{q}, J_{\mathrm{C}-\mathrm{F}}=271.0 \mathrm{~Hz}\right), 121.9,121.8,118.7,116.4,114.0$ $\left(\mathrm{q}, J_{\mathrm{C}-\mathrm{F}}=3.8 \mathrm{~Hz}\right), 112.9\left(\mathrm{q}, J_{\mathrm{C}-\mathrm{F}}=3.6 \mathrm{~Hz}\right) ;{ }^{19} \mathrm{~F}$ NMR $\left(377 \mathrm{MHz}, \mathrm{CDCl}_{3}\right) \delta-63.6$; HRMS (ESI, m/z): calcd for $\mathrm{C}_{17} \mathrm{H}_{12} \mathrm{~F}_{3} \mathrm{~N}_{3} \mathrm{O}[\mathrm{M}+\mathrm{H}]^{+}$: 332.1005; Found: 332.1016.

\section{2-amino-4-fluoro- $N$-(quinolin-8-yl)benzamide (3j):}<smiles>Nc1cc(F)ccc1C(=O)Nc1cccc2cccnc12</smiles>

$3 \mathbf{j}$

Following the general procedure the title compound was isolated by flash chromatography (eluent: petrol ether/ethyl acetate/triethylamine $=80 / 10 / 1$ ) as a white solid in $49 \%$ (58\%) yield $(24.5 \mathrm{mg})$. This compound is known ${ }^{4} .{ }^{1} \mathrm{H}$ NMR $(400 \mathrm{MHz}$, $\left.\mathrm{CDCl}_{3}\right): \delta 10.54(\mathrm{~s}, 1 \mathrm{H}), 8.84-8.75(\mathrm{~m}, 2 \mathrm{H}), 8.16(\mathrm{~d}, J=8.2 \mathrm{~Hz}, 1 \mathrm{H}), 7.75(\mathrm{t}, J=7.3$ $\mathrm{Hz}, 1 \mathrm{H}), 7.60-7.49(\mathrm{~m}, 2 \mathrm{H}), 7.45(\mathrm{dd}, J=7.4,3.8 \mathrm{~Hz}, 1 \mathrm{H}), 6.48(\mathrm{t}, J=8.4 \mathrm{~Hz}, 1 \mathrm{H})$, $6.41(\mathrm{~d}, J=10.8 \mathrm{~Hz}, 1 \mathrm{H}), 5.95(\mathrm{~s}, 2 \mathrm{H}) ;{ }^{13} \mathrm{C} \mathrm{NMR}\left(100 \mathrm{MHz}, \mathrm{CDCl}_{3}\right): \delta 166.9,165.6$ $\left(\mathrm{d}, J_{\mathrm{C}-\mathrm{F}}=248.4 \mathrm{~Hz}\right), 151.7\left(\mathrm{~d}, J_{\mathrm{C}-\mathrm{F}}=12.1 \mathrm{~Hz}\right), 148.2,138.7,136.3,134.6,129.9(\mathrm{~d}$, $\left.J_{\mathrm{C}-\mathrm{F}}=11.2 \mathrm{~Hz}\right), 128.0,127.3,121.6,121.4,116.2,112.6,104.3\left(\mathrm{~d}, J_{\mathrm{C}-\mathrm{F}}=22.6 \mathrm{~Hz}\right)$, $103.1\left(\mathrm{~d}, J_{\mathrm{C}-\mathrm{F}}=24.1 \mathrm{~Hz}\right) ;{ }^{19} \mathrm{~F}$ NMR $\left(377 \mathrm{MHz}, \mathrm{CDCl}_{3}\right): \delta-107.4$.

\section{2-amino-4-chloro- $N$-(quinolin-8-yl)benzamide (3k):}<smiles>Nc1cc(Cl)ccc1C(=O)Nc1cccc2cccnc12</smiles>

$3 \mathbf{k}$

Following the general procedure the title compound was isolated by flash chromatography (eluent: petrol ether/ethyl acetate/triethylamine $=80 / 10 / 1$ ) as a white solid in 54\% (62\%) yield (27.6 mg). This compound is known ${ }^{4} .{ }^{1} \mathrm{H}$ NMR (400 MHz, $\left.\mathrm{CDCl}_{3}\right): \delta 10.58(\mathrm{~s}, 1 \mathrm{H}), 8.84-8.78(\mathrm{~m}, 2 \mathrm{H}), 8.17(\mathrm{~d}, J=8.2 \mathrm{~Hz}, 1 \mathrm{H}), 7.68(\mathrm{~d}, J=8.2$ 
$\mathrm{Hz}, 1 \mathrm{H}), 7.60-744(\mathrm{~m}, 3 \mathrm{H}), 6.74(\mathrm{~d}, J=8.3 \mathrm{~Hz}, 2 \mathrm{H}), 5.86(\mathrm{~s}, 2 \mathrm{H}) ;{ }^{13} \mathrm{C}$ NMR $(100$ $\left.\mathrm{MHz}, \mathrm{CDCl}_{3}\right): \delta 166.9,150.4,148.3,138.7,138.5,136.3,134.5,128.9,128.0,127.3$, $121.7,121.6,116.9,116.7,116.3,114.5$.

2-amino-4-bromo- $N$-(quinolin-8-yl)benzamide (31):<smiles>Nc1cc(Br)ccc1C(=O)Nc1cccc2cccnc12</smiles>

3I

Following the general procedure the title compound was isolated by flash chromatography (eluent: petrol ether/ethyl acetate/triethylamine $=80 / 10 / 1$ ) as a white solid in 47\% (69\%) yield (35.3 mg). This compound is known ${ }^{4} .{ }^{1} \mathrm{H}$ NMR (400 MHz, $\left.\mathrm{CDCl}_{3}\right): \delta 10.57(\mathrm{~s}, 1 \mathrm{H}), 8.84-8.78(\mathrm{~m}, 2 \mathrm{H}), 8.16(\mathrm{~d}, J=8.2 \mathrm{~Hz}, 1 \mathrm{H}), 7.62-7.45(\mathrm{~m}$, $4 \mathrm{H}), 6.94-6.84(\mathrm{~m}, 2 \mathrm{H}), 5.84(\mathrm{~s}, 2 \mathrm{H}) ;{ }^{13} \mathrm{C} \mathrm{NMR}\left(100 \mathrm{MHz}, \mathrm{CDCl}_{3}\right): \delta 166.9,150.4$, 148.2 , 138.7, 136.3, 134.4, 128.9, 127.9, 127.3, 127.0, 121.7, 121.6, 119.7, 116.3, 114.9 .

\section{2-amino-4-nitro- $N$-(quinolin-8-yl)benzamide (3m):}<smiles>Nc1cc([N+](=O)[O-])ccc1C(=O)Nc1cccc2cccnc12</smiles>

Following the general procedure the title compound was isolated by flash chromatography (eluent: petrol ether/ethyl acetate/triethylamine $=20 / 10 / 1$ ) as a yellow solid in $58 \%$ (71\%) yield (32.8 mg), mp 218-219 ${ }^{\circ} \mathrm{C} .{ }^{1} \mathrm{H}$ NMR (400 MHz, $\left.\mathrm{CDCl}_{3}\right): \delta 10.59(\mathrm{~s}, 1 \mathrm{H}), 8.93(\mathrm{~s}, 1 \mathrm{H}), 8.64(\mathrm{~d}, J=7.5 \mathrm{~Hz}, 1 \mathrm{H}), 8.43(\mathrm{~d}, J=8.2 \mathrm{~Hz}$, $1 \mathrm{H}), 7.89(\mathrm{~d}, J=8.6,1 \mathrm{H}), 7.77-7.58(\mathrm{~m}, 4 \mathrm{H}), 7.41(\mathrm{~d}, J=8.5 \mathrm{~Hz}, 1 \mathrm{H}), 6.91(\mathrm{~s}, 2 \mathrm{H})$; ${ }^{13} \mathrm{C} \mathrm{NMR}\left(100 \mathrm{MHz}, \mathrm{CDCl}_{3}\right): \delta 165.6,150.3,150.0,149.4,138.6,136.9,134.0,129.7$, 128.0, 127.1 122.8, 122.5, 120.2, 117.3, 111.1, 109.3; HRMS (ESI, m/z): calcd for $\mathrm{C}_{16} \mathrm{H}_{12} \mathrm{~N}_{4} \mathrm{O}_{3}[\mathrm{M}+\mathrm{H}]^{+}$: 309.0982 ; Found: 309.0982.

\section{4-amino- $N$-(quinolin-8-yl)-[1,1'-biphenyl]-3-carboxamide (3n):}<smiles>Nc1ccc(-c2ccccc2)cc1C(=O)Nc1cccc2cccnc12</smiles>

3n

Following the general procedure the title compound was isolated by flash chromatography (eluent: petrol ether/ethyl acetate/triethylamine $=40 / 10 / 1$ ) as a yellow solid in $56 \%(63 \%)$ yield $(32.0 \mathrm{mg})$. This compound is known ${ }^{4}$. ${ }^{1} \mathrm{H}$ NMR (400 
$\left.\mathrm{MHz}, \mathrm{CDCl}_{3}\right): \delta 10.68(\mathrm{~s}, 1 \mathrm{H}), 8.88(\mathrm{~d}, J=7.5 \mathrm{~Hz}, 1 \mathrm{H}), 8.83(\mathrm{~d}, J=3.7 \mathrm{~Hz}, 1 \mathrm{H}), 8.18$ $(\mathrm{d}, J=8.2 \mathrm{~Hz}, 1 \mathrm{H}), 7.99(\mathrm{~s}, 1 \mathrm{H}), 7.66-7.50(\mathrm{~m}, 5 \mathrm{H}), 7.47(\mathrm{t}, J=6.9 \mathrm{~Hz}, 3 \mathrm{H}), 7.34$ (t, $J$ $=7.2 \mathrm{~Hz}, 1 \mathrm{H}), 6.84(\mathrm{~d}, J=8.4 \mathrm{~Hz}, 1 \mathrm{H}), 5.74(\mathrm{~s}, 2 \mathrm{H}) ;{ }^{13} \mathrm{C} \mathrm{NMR}\left(100 \mathrm{MHz}, \mathrm{CDCl}_{3}\right): \delta$ $167.7,148.6,148.3,140.6,138.8,136.3,134.7,131.5,129.9,128.8,128.0,127.3$, $126.5,126.4,126.1,121.6,121.5,117.9,116.8,116.4$.

\section{2-amino- $N$-(quinolin-8-yl)-5-(trifluoromethyl)benzamide (3o):}<smiles>Nc1ccc(C(F)(F)F)cc1C(=O)Nc1cccc2cccnc12</smiles>

30

Following the general procedure the title compound was isolated by flash chromatography (eluent: petrol ether/ethyl acetate/triethylamine $=80 / 10 / 1$ ) as a white solid in $76 \%(82 \%)$ yield $(40.7 \mathrm{mg})$. This compound is known ${ }^{4} .{ }^{1} \mathrm{H}$ NMR $(400 \mathrm{MHz}$, $\left.\mathrm{CDCl}_{3}\right): \delta 10.61(\mathrm{~s}, 1 \mathrm{H}), 8.85(\mathrm{~d}, J=3.9 \mathrm{~Hz}, 1 \mathrm{H}), 8.80(\mathrm{~d}, J=7.1 \mathrm{~Hz}, 1 \mathrm{H}), 8.17(\mathrm{~d}, J$ $=8.2 \mathrm{~Hz}, 1 \mathrm{H}), 7.98(\mathrm{~s}, 1 \mathrm{H}), 7.60-7.52(\mathrm{~m}, 2 \mathrm{H}), 7.47(\mathrm{dd}, J=7.4,5.6 \mathrm{~Hz}, 2 \mathrm{H}), 6.77(\mathrm{~d}$, $J=8.6 \mathrm{~Hz}, 1 \mathrm{H}), 6.09$ (s, 2H); ${ }^{13} \mathrm{C} \mathrm{NMR}\left(100 \mathrm{MHz}, \mathrm{CDCl}_{3}\right): \delta 166.6,151.8,148.5$, $138.7,136.3,134.3,129.3\left(\mathrm{q}, J_{\mathrm{C}-\mathrm{F}}=3.2 \mathrm{~Hz}\right), 128.0,127.2,125.2\left(\mathrm{q}, J_{\mathrm{C}-\mathrm{F}}=3.9 \mathrm{~Hz}\right)$, $124.4\left(\mathrm{q}, J_{\mathrm{C}-\mathrm{F}}=269.0 \mathrm{~Hz}\right), 121.9,121.7,118.3\left(\mathrm{q}, J_{\mathrm{C}-\mathrm{F}}=32.8 \mathrm{~Hz}\right), 117.2,116.5,115.3$; ${ }^{19} \mathrm{~F}$ NMR (377 MHz, $\left.\mathrm{CDCl}_{3}\right): \delta-61.3$.

\section{5-acetyl-2-amino- $N$-(quinolin-8-yl)benzamide (3p):}<smiles>CC(=O)c1ccc(N)c(C(=O)Nc2cccc3cccnc23)c1</smiles>

Following the general procedure the title compound was isolated by flash chromatography (eluent: petrol ether/ethyl acetate/triethylamine $=80 / 10 / 1$ ) as a white solid in 52\% (60\%) yield (27.5 mg), mp 212-213 $\left.{ }^{\circ} \mathrm{C} .{ }^{1} \mathrm{H} \mathrm{NMR} \mathrm{(400} \mathrm{MHz}, \mathrm{CDCl}_{3}\right): \delta$ $10.71(\mathrm{~s}, 1 \mathrm{H}), 8.86(\mathrm{~s}, 1 \mathrm{H}), 8.80(\mathrm{~d}, J=6.9 \mathrm{~Hz}, 1 \mathrm{H}), 8.47(\mathrm{~s}, 1 \mathrm{H}), 8.19(\mathrm{~d}, J=8.2 \mathrm{~Hz}$, $1 \mathrm{H}), 7.89(\mathrm{~d}, J=8.6,1 \mathrm{H}), 7.62-7.46(\mathrm{~m}, 3 \mathrm{H}), 6.72(\mathrm{~d}, J=8.3 \mathrm{~Hz}, 1 \mathrm{H}), 6.31(\mathrm{~s}, 2 \mathrm{H})$, $2.60(\mathrm{~s}, 3 \mathrm{H}) ;{ }^{13} \mathrm{C} \mathrm{NMR}\left(100 \mathrm{MHz}, \mathrm{CDCl}_{3}\right): \delta 195.6,167.0,153.2,148.5,138.8,136.3$, 134.4, 133.0, 129.4, 128.0, 127.3, 126.2, 121.8, 121.8, 116.6, 116.4, 115.0, 26.0; HRMS (ESI, m/z): calcd for $\mathrm{C}_{18} \mathrm{H}_{15} \mathrm{~N}_{3} \mathrm{O}_{2}[\mathrm{M}+\mathrm{H}]^{+}: 306.1237$; Found: 306.1252 .

\section{2-amino- $N$-(quinolin-8-yl)-6-(trifluoromethyl)benzamide (3q)}


<smiles>Nc1cccc(C(F)(F)F)c1C(=O)Nc1cccc2cccnc12</smiles>

$3 q$

Following the general procedure the title compound was isolated by flash chromatography (eluent: petrol ether/ethyl acetate/triethylamine $=80 / 10 / 1$ ) as a white solid in $40 \%$ (53\%) yield $(26.3 \mathrm{mg}), \mathrm{mp} 140-141{ }^{\circ} \mathrm{C} .{ }^{1} \mathrm{H}$ NMR $\left(400 \mathrm{MHz}, \mathrm{CDCl}_{3}\right): \delta$ $10.19(\mathrm{~s}, 1 \mathrm{H}), 8.93(\mathrm{~d}, J=3.6 \mathrm{~Hz}, 1 \mathrm{H}), 8.75(\mathrm{~s}, 1 \mathrm{H}), 8.16(\mathrm{~d}, J=8.1 \mathrm{~Hz}, 1 \mathrm{H})$, 7.65-7.52 (m, 2H), $7.44(\mathrm{dd}, J=8.0 \mathrm{~Hz}, 4.0 \mathrm{~Hz}, 1 \mathrm{H}), 7.31(\mathrm{t}, J=7.6 \mathrm{~Hz}, 1 \mathrm{H}), 7.11(\mathrm{~d}$, $J=7.6 \mathrm{~Hz}, 1 \mathrm{H}), 6.93(\mathrm{~d}, J=8.0 \mathrm{~Hz}, 1 \mathrm{H}), 4.45(\mathrm{~s}, 2 \mathrm{H}) ;{ }^{13} \mathrm{C} \mathrm{NMR}\left(100 \mathrm{MHz}, \mathrm{CDCl}_{3}\right)$ : $\delta 165.4,148.4,145.7,138.5,136.3,134.2,130.3,130.0,127.2,122.5,121.7,120.1$ (q, $\left.J_{\mathrm{C}-\mathrm{F}}=2.2 \mathrm{~Hz}\right), 119.8,117.0,115.6\left(\mathrm{q}, J_{\mathrm{C}-\mathrm{F}}=5.2 \mathrm{~Hz}\right) ;{ }^{19} \mathrm{~F}$ NMR $\left(377 \mathrm{MHz}, \mathrm{CDCl}_{3}\right): \delta$ -58.3; HRMS (ESI, m/z): calcd for $\mathrm{C}_{17} \mathrm{H}_{12} \mathrm{~F}_{3} \mathrm{~N}_{3} \mathrm{O}[\mathrm{M}+\mathrm{H}]^{+}$: 332.1005; Found: 332.1017 .

\section{2-amino- $N$-(quinolin-8-yl)-1-naphthamide (3r)}<smiles>Nc1ccc2ccccc2c1C(=O)Nc1cccc2cccnc12</smiles>

$3 r$

Following the general procedure the title compound was isolated by flash chromatography (eluent: petrol ether/ethyl acetate/triethylamine $=80 / 10 / 1$ ) as a white solid in 78\% (80\%) yield (37.6 mg), mp 207-208 ${ }^{\circ} \mathrm{C} .{ }^{1} \mathrm{H}$ NMR (400 MHz, DMSO- $\left.d_{6}\right)$ : $10.24(\mathrm{~s}, 1 \mathrm{H}), 8.86(\mathrm{~d}, J=6.5 \mathrm{~Hz}, 1 \mathrm{H}), 8.79(\mathrm{~d}, J=2.4 \mathrm{~Hz}, 1 \mathrm{H}), 8.43(\mathrm{~d}, J=8.1 \mathrm{~Hz}$, $1 \mathrm{H}), 7.86(\mathrm{~d}, J=8.4 \mathrm{~Hz}, 1 \mathrm{H}), 7.81-7.66(\mathrm{~m}, 4 \mathrm{H}), 7.60(\mathrm{dd}, J=8.0,4.0 \mathrm{~Hz}, 1 \mathrm{H}), 7.38$ $(\mathrm{t}, J=7.4 \mathrm{~Hz}, 1 \mathrm{H}), 7.21(\mathrm{t}, J=7.2 \mathrm{~Hz}, 1 \mathrm{H}), 7.14(\mathrm{~d}, J=8.9 \mathrm{~Hz}, 1 \mathrm{H}), 6.03(\mathrm{~s}, 2 \mathrm{H}) ;{ }^{13} \mathrm{C}$ NMR (100 MHz, DMSO- $\left.d_{6}\right): \delta 166.9,149.1,145.1,138.3,136.8,134.5,131.6,131.2$, 128.4, 128.0, 127.4, 127.2, 126.5, 122.4, 122.4, 122.3, 121.7, 119.2, 117.1, 111.1; HRMS (ESI, m/z): calcd for $\mathrm{C}_{20} \mathrm{H}_{15} \mathrm{~N}_{3} \mathrm{O}[\mathrm{M}+\mathrm{H}]^{+}: 314.1288$; Found: 314.1301 .

\section{3-amino- $N$-(quinolin-8-yl)isonicotinamide (3s)}<smiles>Nc1cnccc1C(=O)Nc1cccc2cccnc12</smiles>

3s

Following the general procedure the title compound was isolated by flash chromatography (eluent: petrol ether/ethyl acetate/triethylamine $=40 / 10 / 1$ ) as a yellow solid in $33 \%$ (48\%) yield $\left(19.0 \mathrm{mg}\right.$ ). This compound is known ${ }^{4} .{ }^{1} \mathrm{H}$ NMR (400 
$\left.\mathrm{MHz}, \mathrm{CDCl}_{3}\right): \delta 10.73(\mathrm{~s}, 1 \mathrm{H}), 8.90-8.72(\mathrm{~m}, 2 \mathrm{H}), 8.23(\mathrm{~s}, 1 \mathrm{H}), 8.17(\mathrm{~d}, J=8.2 \mathrm{~Hz}$, $1 \mathrm{H}), 8.05(\mathrm{~d}, J=4.0 \mathrm{~Hz}, 1 \mathrm{H}), 7.60-7.45(\mathrm{~m}, 4 \mathrm{H}), 5.74(\mathrm{~s}, 2 \mathrm{H}) ;{ }^{13} \mathrm{C} \mathrm{NMR}(100 \mathrm{MHz}$, $\left.\mathrm{CDCl}_{3}\right): \delta 165.7,148.4,144.0,141.1,138.6,137.7,136.4,134.0,127.9,127.3,122.1$, $121.8,120.6,119.8,116.5$.

\section{2-amino-4-methyl- $N$-(quinolin-8-yl)-5-(trifluoromethyl)benzamide (3t)}<smiles>Cc1cc(N)c(C(=O)Nc2cccc3cccnc23)cc1C(F)(F)F</smiles>

$3 t$

Following the general procedure the title compound was isolated by flash chromatography (eluent: petrol ether/ethyl acetate/triethylamine $=80 / 10 / 1$ ) as a white solid in 66\% (73\%) yield (37.8 mg), mp 146-147 ${ }^{\circ} \mathrm{C} .{ }^{1} \mathrm{H}$ NMR (400 MHz, $\left.\mathrm{CDCl}_{3}\right): \delta$ $10.60(\mathrm{~s}, 1 \mathrm{H}), 8.86(\mathrm{~d}, J=1.6 \mathrm{~Hz}, 1 \mathrm{H}), 8.79(\mathrm{~d}, J=7.0 \mathrm{~Hz}, 1 \mathrm{H}), 8.18(\mathrm{~d}, J=8.1 \mathrm{~Hz}$, $1 \mathrm{H}), 7.98(\mathrm{~s}, 1 \mathrm{H}), 7.61-7.51(\mathrm{~m}, 2 \mathrm{H}), 7.48(\mathrm{dd}, J=4.9,3.2 \mathrm{~Hz}, 1 \mathrm{H}), 6.59$ (s, 1H), 6.05 (s, 2H), $2.42(\mathrm{~s}, 3 \mathrm{H}) ;{ }^{13} \mathrm{C} \mathrm{NMR}\left(100 \mathrm{MHz}, \mathrm{CDCl}_{3}\right): \delta 166.7,151.7,148.4,141.4$, 138.8, 136.3, 134.4, 128.0, 127.3, 126.0 (q, $\left.J_{\mathrm{C}-\mathrm{F}}=5.7 \mathrm{~Hz}\right), 124.8\left(\mathrm{q}, J_{\mathrm{C}-\mathrm{F}}=270.1 \mathrm{~Hz}\right)$, $121.7,121.7,119.8,117.1$ (q, $\left.J_{\mathrm{C}-\mathrm{F}}=30.1 \mathrm{~Hz}\right), 116.3,112.5,19.4 ;{ }^{19} \mathrm{~F}$ NMR $(377 \mathrm{MHz}$, $\mathrm{CDCl}_{3}$ ): $\delta$-60.0; HRMS (ESI, m/z): calcd for $\mathrm{C}_{18} \mathrm{H}_{14} \mathrm{~F}_{3} \mathrm{~N}_{3} \mathrm{O}[\mathrm{M}+\mathrm{H}]^{+}: 346.1162$; Found: 346.1175 .

\section{2-amino-4,5-dichloro- $N$-(quinolin-8-yl)benzamide (3u)}<smiles>Nc1cc(Cl)c(Cl)cc1C(=O)Nc1cccc2cccnc12</smiles>

3u

Following the general procedure the title compound was isolated by flash chromatography (eluent: petrol ether/ethyl acetate/triethylamine $=40 / 10 / 1$ ) as a white solid in 53\% (61\%) yield (30.3 mg). This compound is known ${ }^{4} .{ }^{1} \mathrm{H}$ NMR (400 MHz, $\left.\mathrm{CDCl}_{3}\right): \delta 10.52(\mathrm{~s}, 1 \mathrm{H}), 8.87(\mathrm{~s}, 1 \mathrm{H}), 8.79(\mathrm{~d}, J=6.4 \mathrm{~Hz}, 1 \mathrm{H}), 8.19(\mathrm{~d}, J=8.1 \mathrm{~Hz}$, $1 \mathrm{H}), 7.80(\mathrm{~s}, 1 \mathrm{H}), 7.64-7.54(\mathrm{~m}, 2 \mathrm{H}), 7.50(\mathrm{dd}, J=7.6,3.8 \mathrm{~Hz}, 1 \mathrm{H}), 6.85$ (s, 1H), 5.77 $(\mathrm{s}, 2 \mathrm{H}) ;{ }^{13} \mathrm{C}$ NMR $\left(100 \mathrm{MHz}, \mathrm{CDCl}_{3}\right): \delta 165.8,148.5,148.5,138.7,136.5,136.4$, $134.2,128.9,128.0,127.3,121.9,121.8,119.3,118.3,116.5,116.0$.

\section{2-amino-4-(pyridin-2-yl)- $N$-(quinolin-8-yl)benzamide (3w)}


<smiles>Nc1cc(-c2ccccn2)ccc1C(=O)Nc1cccc2cccnc12</smiles>

Following the general procedure the title compound was isolated by flash chromatography (eluent: petrol ether/ethyl acetate/triethylamine $=20 / 10 / 1$ ) as a white solid in 51\% (65\%) yield (41.3 mg), mp 184-185 $\left.{ }^{\circ} \mathrm{C} .{ }^{1} \mathrm{H} \mathrm{NMR} \mathrm{(400} \mathrm{MHz}, \mathrm{CDCl}_{3}\right): \delta$ $10.68(\mathrm{~s}, 1 \mathrm{H}), 8.90-8.80(\mathrm{~m}, 2 \mathrm{H}), 8.69(\mathrm{~d}, J=3.8 \mathrm{~Hz}, 1 \mathrm{H}), 8.16(\mathrm{~d}, J=8.3,1 \mathrm{H}), 7.86$ $(\mathrm{d}, J=8.2,1 \mathrm{H}), 7.78-7.70(\mathrm{~m}, 2 \mathrm{H}), 7.60-7.48(\mathrm{~m}, 2 \mathrm{H}), 7.49-7.41(\mathrm{~m}, 2 \mathrm{H}), 7.35(\mathrm{~d}, J=$ $8.2,1 \mathrm{H}), 7.25(\mathrm{~d}, J=6.8,1 \mathrm{H}), 5.86(\mathrm{~s}, 2 \mathrm{H}) ;{ }^{13} \mathrm{C} \mathrm{NMR}\left(100 \mathrm{MHz}, \mathrm{CDCl}_{3}\right): \delta 167.4$, $156.4,149.8,149.7,148.2,143.2,138.8,136.8,136.3,134.7,128.2,128.0,127.4$, 122.7, 121.6, 121.4, 120.8, 116.4, 116.3, 115.7, 115.2; HRMS (ESI, m/z): calcd for $\mathrm{C}_{21} \mathrm{H}_{16} \mathrm{~N}_{4} \mathrm{O}[\mathrm{M}+\mathrm{H}]^{+}:$341.1397; Found: 341.1406.

\section{2-amino-4-(2H-pyrrol-2-yl)- $N$-(quinolin-8-yl)benzamide (3x)}<smiles>Nc1cc(-n2cccn2)ccc1C(=O)Nc1cccc2cccnc12</smiles>

Following the general procedure the title compound was isolated by flash chromatography (eluent: petrol ether/ethyl acetate/triethylamine $=20 / 10 / 1$ ) as a white solid in 59\% (68\%) yield (42.0 mg), mp 195-196 ${ }^{\circ} \mathrm{C} .{ }^{1} \mathrm{H}$ NMR (400 MHz, DMSO- $\left.d_{6}\right)$ : $\delta 10.56(\mathrm{~s}, 1 \mathrm{H}), 9.00-8.95(\mathrm{~m}, 1 \mathrm{H}), 8.69(\mathrm{~d}, J=7.4 \mathrm{~Hz}, 1 \mathrm{H}), 8.50-8.43(\mathrm{~m}, 2 \mathrm{H}), 7.83$ $(\mathrm{d}, J=8.7 \mathrm{~Hz}, 1 \mathrm{H}), 7.79(\mathrm{~s}, 1 \mathrm{H}), 7.75-7.61(\mathrm{~m}, 3 \mathrm{H}), 7.37(\mathrm{~s}, 1 \mathrm{H}), 7.16(\mathrm{~d}, J=8.5 \mathrm{~Hz}$, $1 \mathrm{H}), 6.83(\mathrm{~s}, 2 \mathrm{H}), 6.58(\mathrm{~s}, 1 \mathrm{H}) ;{ }^{13} \mathrm{C}$ NMR (100 MHz, DMSO-d 6$): \delta 166.4,151.4$, $149.2,142.8,141.5,138.4,136.9,134.4,129.4,128.0,128.0,127.2,122.4,122.0$, 116.5, 112.4, 108.3, 105.8, 105.6; HRMS (ESI, m/z): calcd for $\mathrm{C}_{19} \mathrm{H}_{15} \mathrm{~N}_{5} \mathrm{O}[\mathrm{M}+\mathrm{H}]^{+}$: 330.1349; Found: 330.1364.

\section{References}

1. A. M. Suess, M. Z. Ertem, C. J. Cramer, S. S. Stahl, J. Am. Chem. Soc. 2013, 135, 9797-9804.

2. (a) M. Shang, S.-Z. Sun, H.-X. Dai, J.-Q. Yu, J. Am. Chem. Soc. 2014, 136, 3354 -3357; (b) Q. Zhang, K. Chen, W. Rao, Y. Zhang, F.-J. Chen, B.-F. Shi, Angew. Chem. Int. Ed. 2013, 52, 13588-13592; (c) X.-Q. Hao, L.-J. Chen, B. Ren, L.-Y. Li, X.-Y. Yang, J.-F. Gong, J.-L. Niu, M.-P. Song, Org. Lett. 2014, 16, 1104-1107; (d) H. Shiota, Y. Ano, Y. Aihara, Y. Fukumoto, N. Chatani, J. Am.Chem. Soc. 2011, 133, 14952-14955; (e) L. D. Tran, I. Popov, O. Daugulis, J. Am. Chem. Soc. 2012, 134, 18237-18240; (f) T. Truong, K. Klimovica, O. Daugulis, J. Am. Chem. Soc. 2013, 135, 9342-9345; (g) L. D. Tran, J. Roane, O. Daugulis, Angew. Chem., Int. 
Ed. 2013, 52, 6043-6046; (h) M. Nishino, K. Hirano, T. Satoh, M. Miura, Angew. Chem. Int. Ed. 2013, 52, 4457-4461; (i) J. Roane, O. Daugulis, J. Am. Chem. Soc. 2016, 138, 4601-4607; (j) K. Shibata, N. Chatani, Org. Lett. 2014, 16, 5148-5151.

3. M. Shang, M.-M. Wang, T. G. Sanit-Denis, M.-H. Li, H.-X. Dai, J.-Q. Yu, Angew. Chem. Int. Ed. 2017, 56, 5317-5321.

4. (a) L. Yu, X. Chen, D. Liu, L. Hu, Y. Yu, H. Huang, Z. Tan, Q. Gui, Adv. Synth. Catal. 2018, 360, 1346-1351; (b) Z. Li, H. Yu, C. Bolm, Angew. Chem. Int. Ed. 2017, 56, 9532-9535. 


\section{NMR spectra}

${ }^{1} \mathrm{H}$ NMR Spectrum of $\mathbf{1} \mathbf{w}$

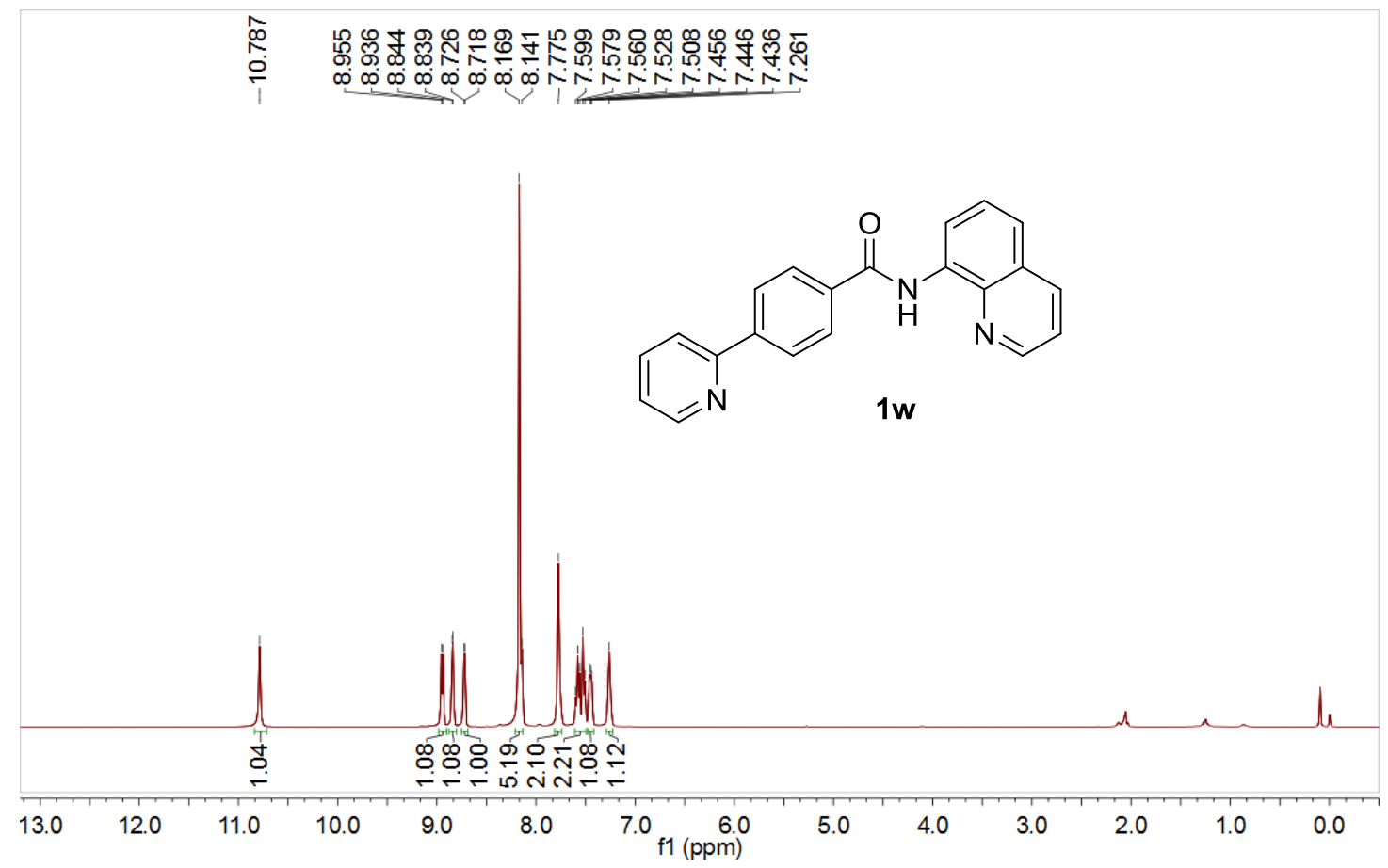

${ }^{13} \mathrm{C}$ NMR Spectrum of $\mathbf{1 w}$

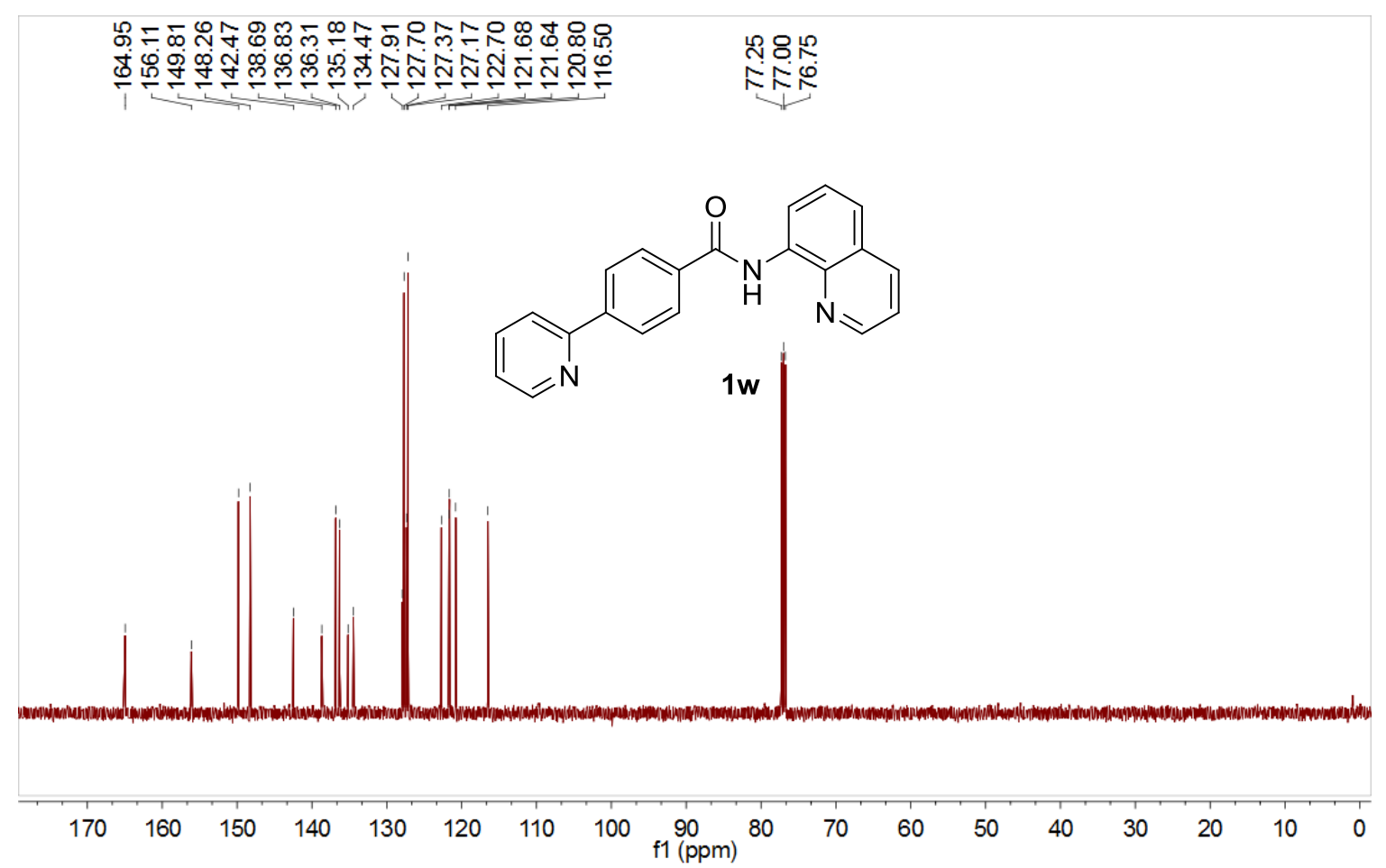


${ }^{1} \mathrm{H}$ NMR Spectrum of $\mathbf{1 x}$

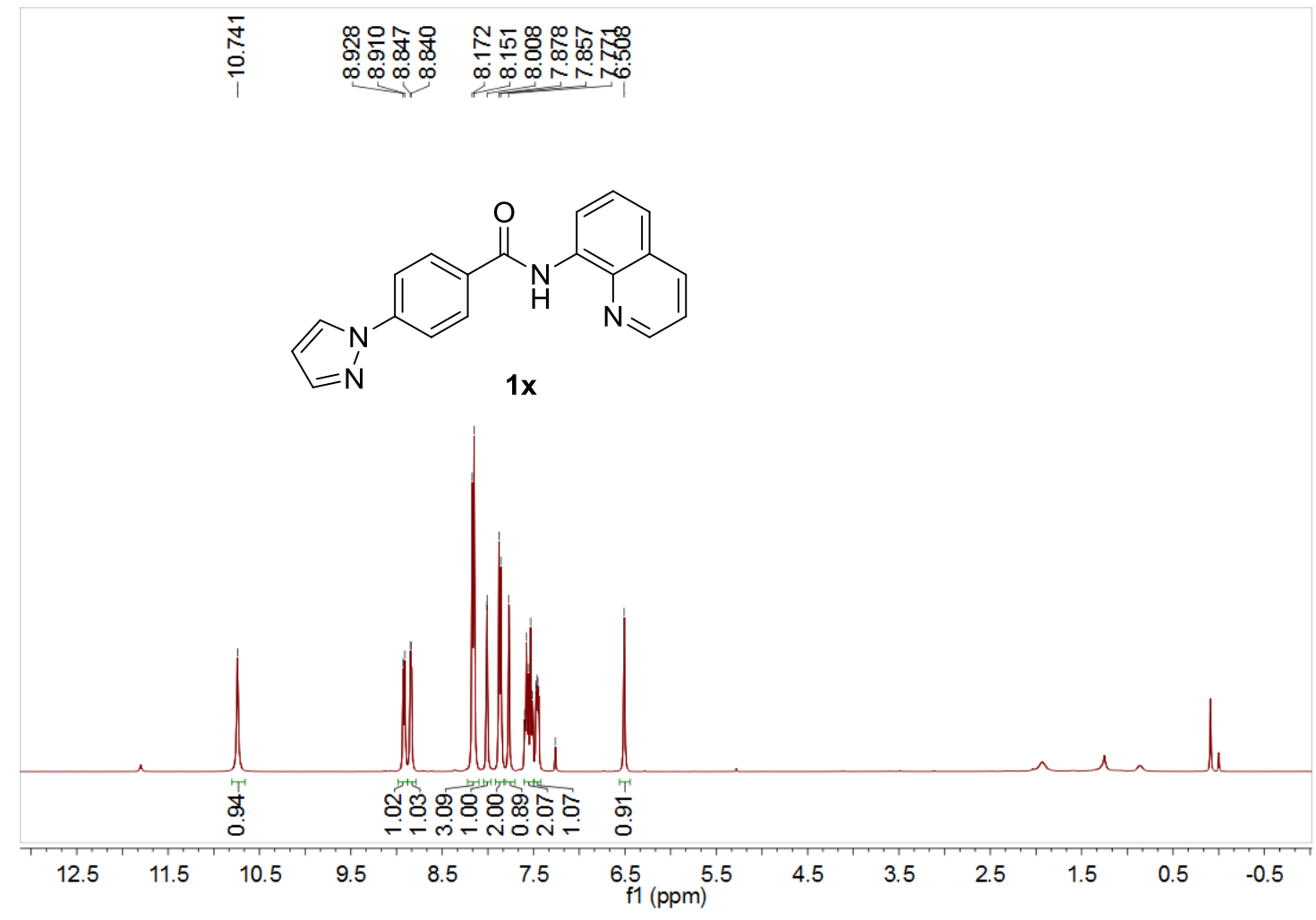

${ }^{13} \mathrm{C}$ NMR Spectrum of $\mathbf{1 x}$

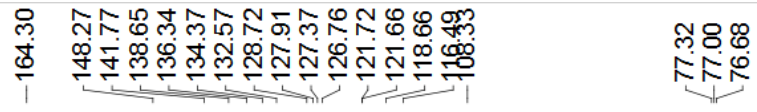<smiles>[X]c1ccc(-n2cccn2)cc1C(=O)Nc1cccc2cccnc12</smiles>

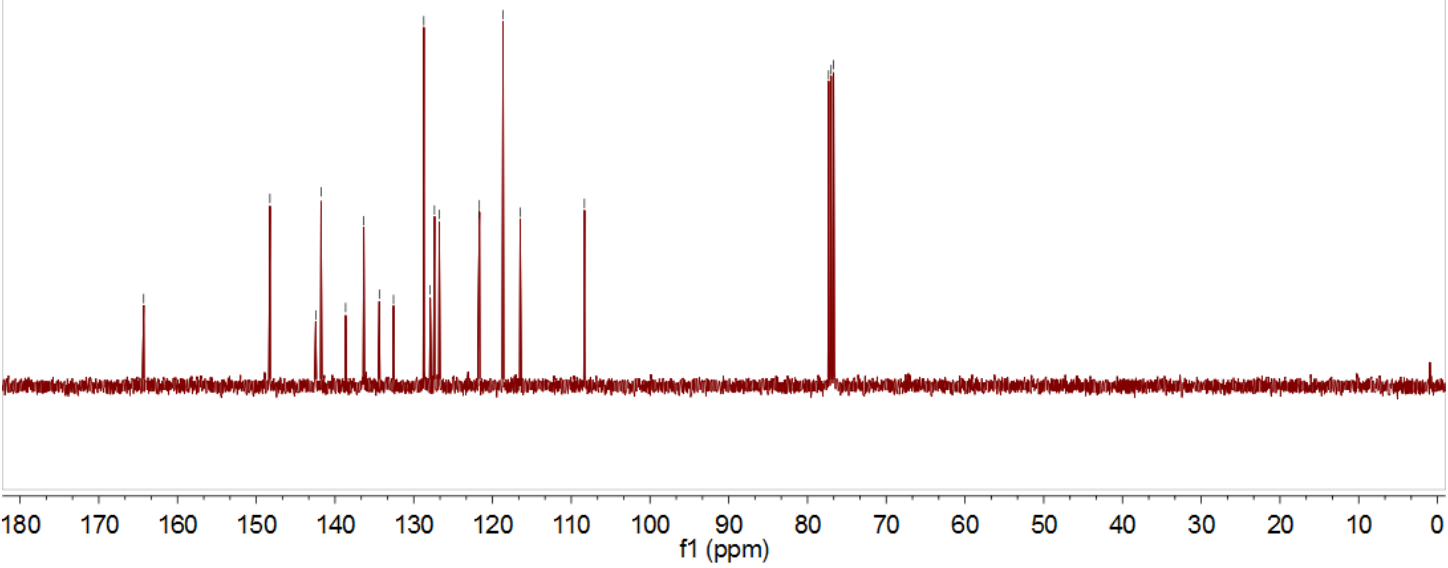


${ }^{1} \mathrm{H}$ NMR Spectrum of $\mathbf{3 a c}$

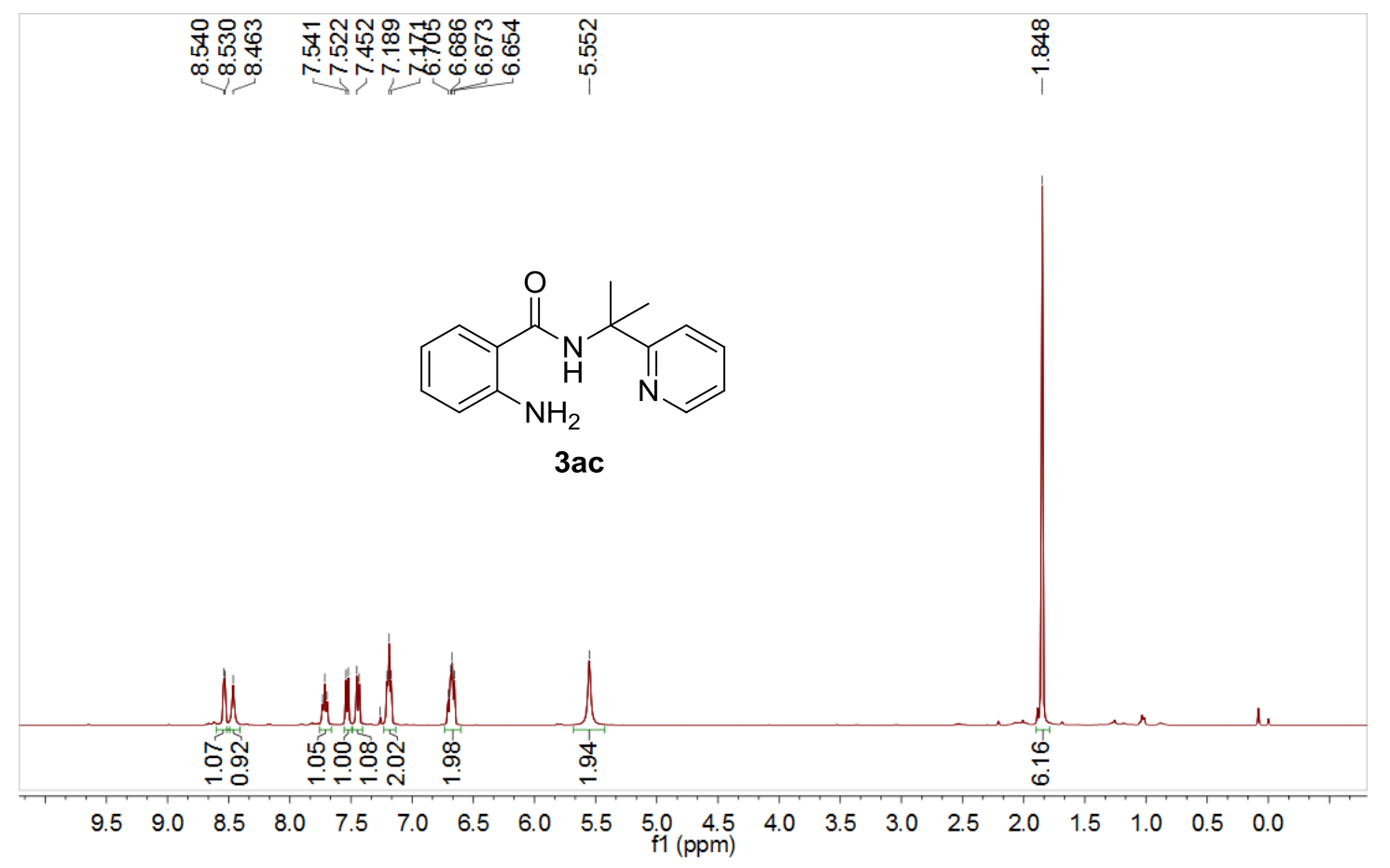

${ }^{13} \mathrm{C}$ NMR Spectrum of $\mathbf{3 a c}$

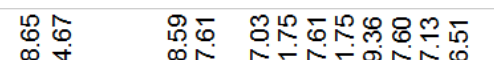

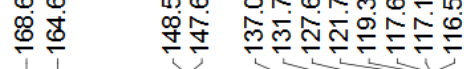

도유

$\underset{\substack{1 \\ 0}}{\stackrel{1}{\pi}}$

$\underbrace{N_{3 H_{2}}}_{3 a c}$

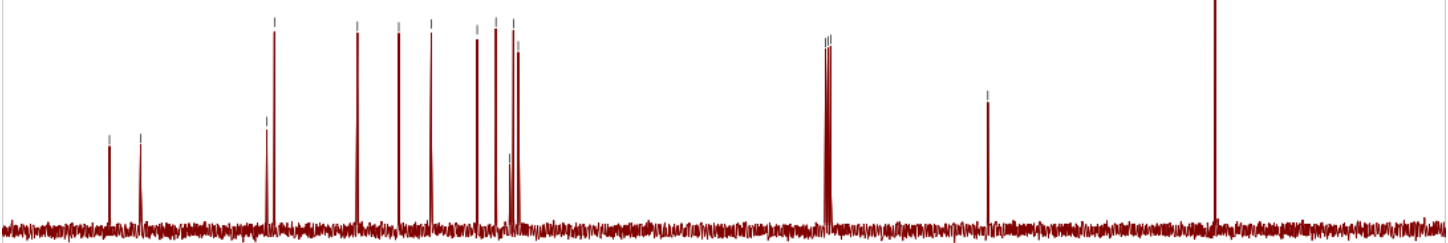

180

150

130 
${ }^{1} \mathrm{H}$ NMR Spectrum of $\mathbf{3 a}$

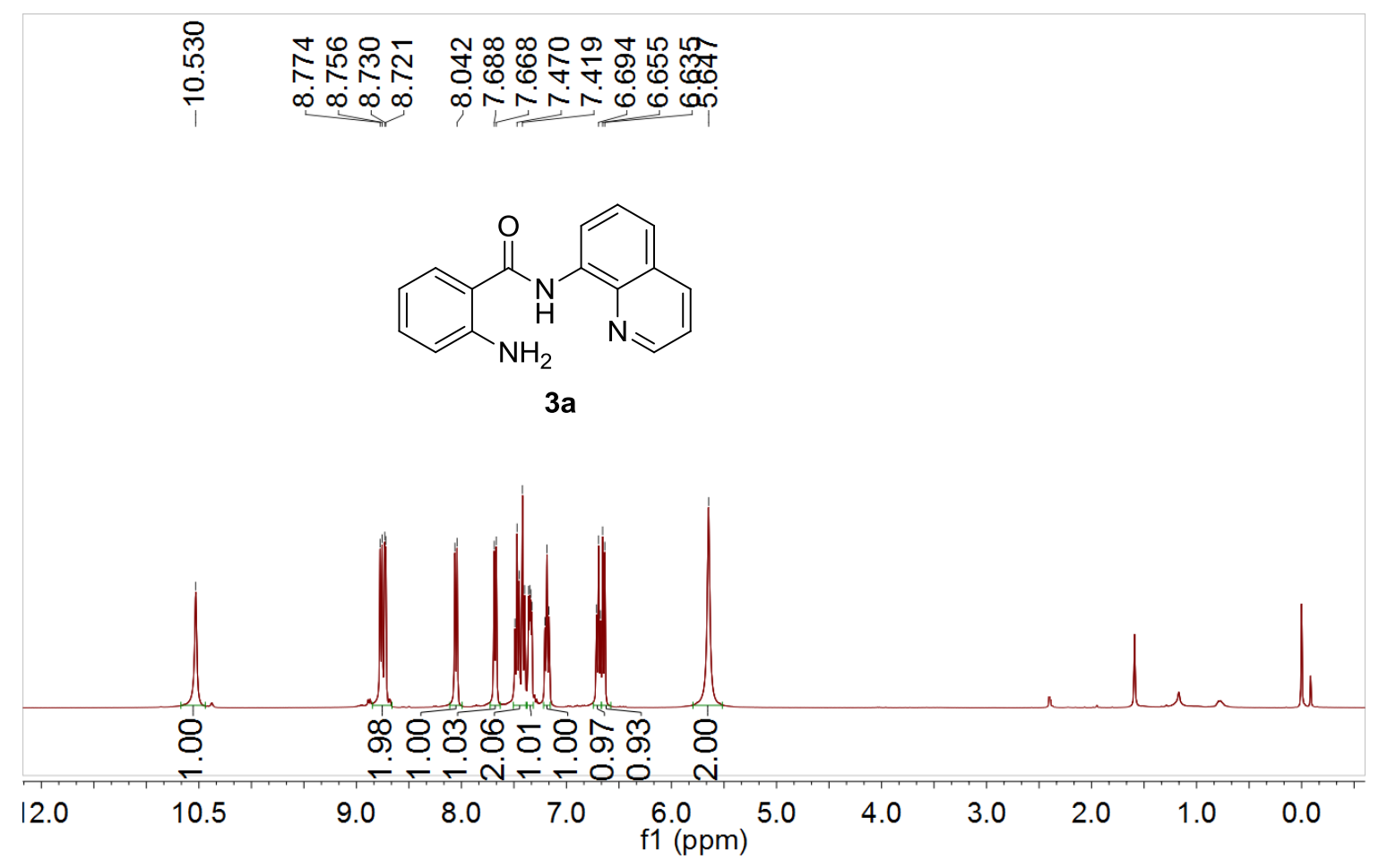

${ }^{13} \mathrm{C}$ NMR Spectrum of $\mathbf{3 a}$

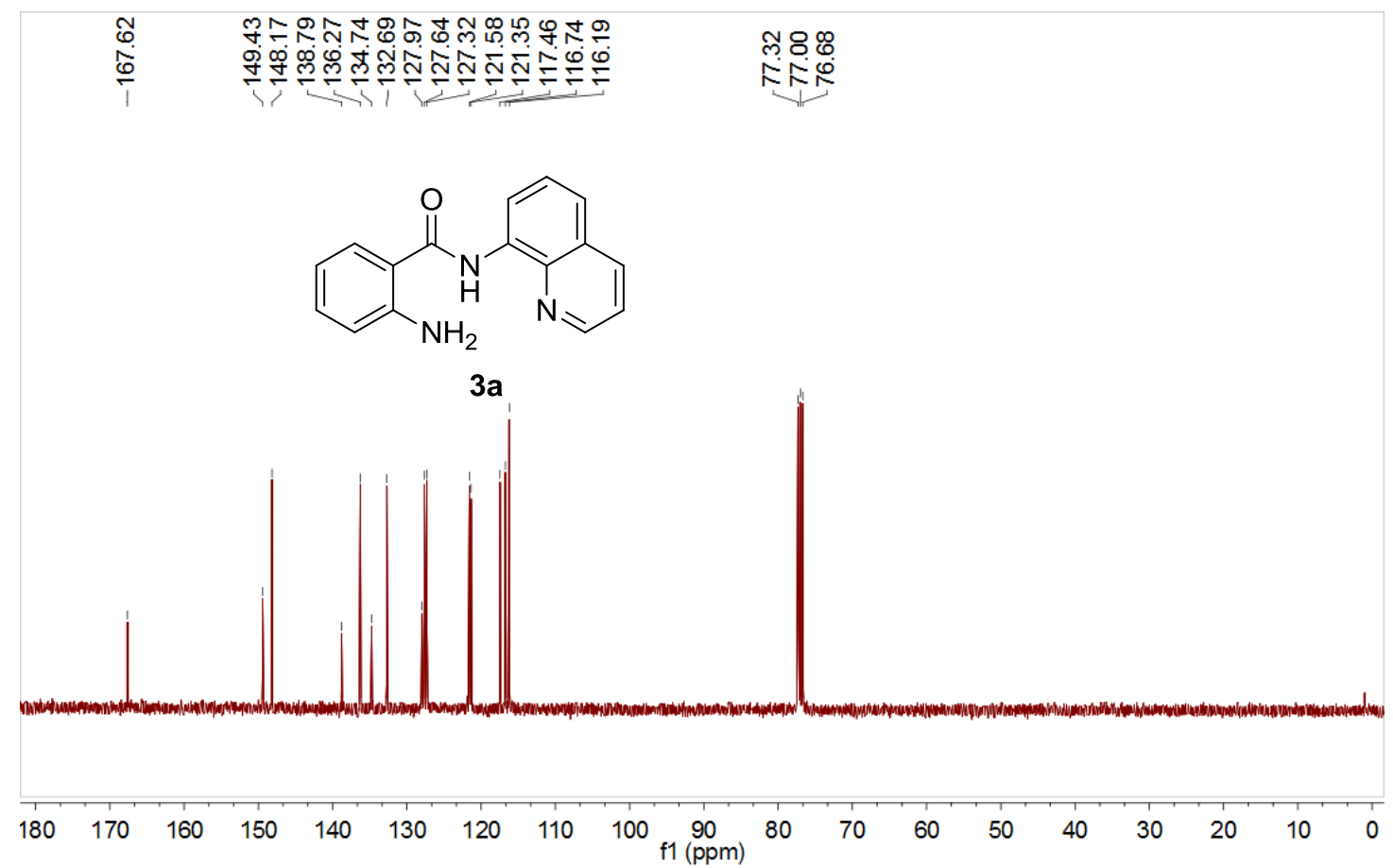


${ }^{1} \mathrm{H}$ NMR Spectrum of $\mathbf{3 b}$

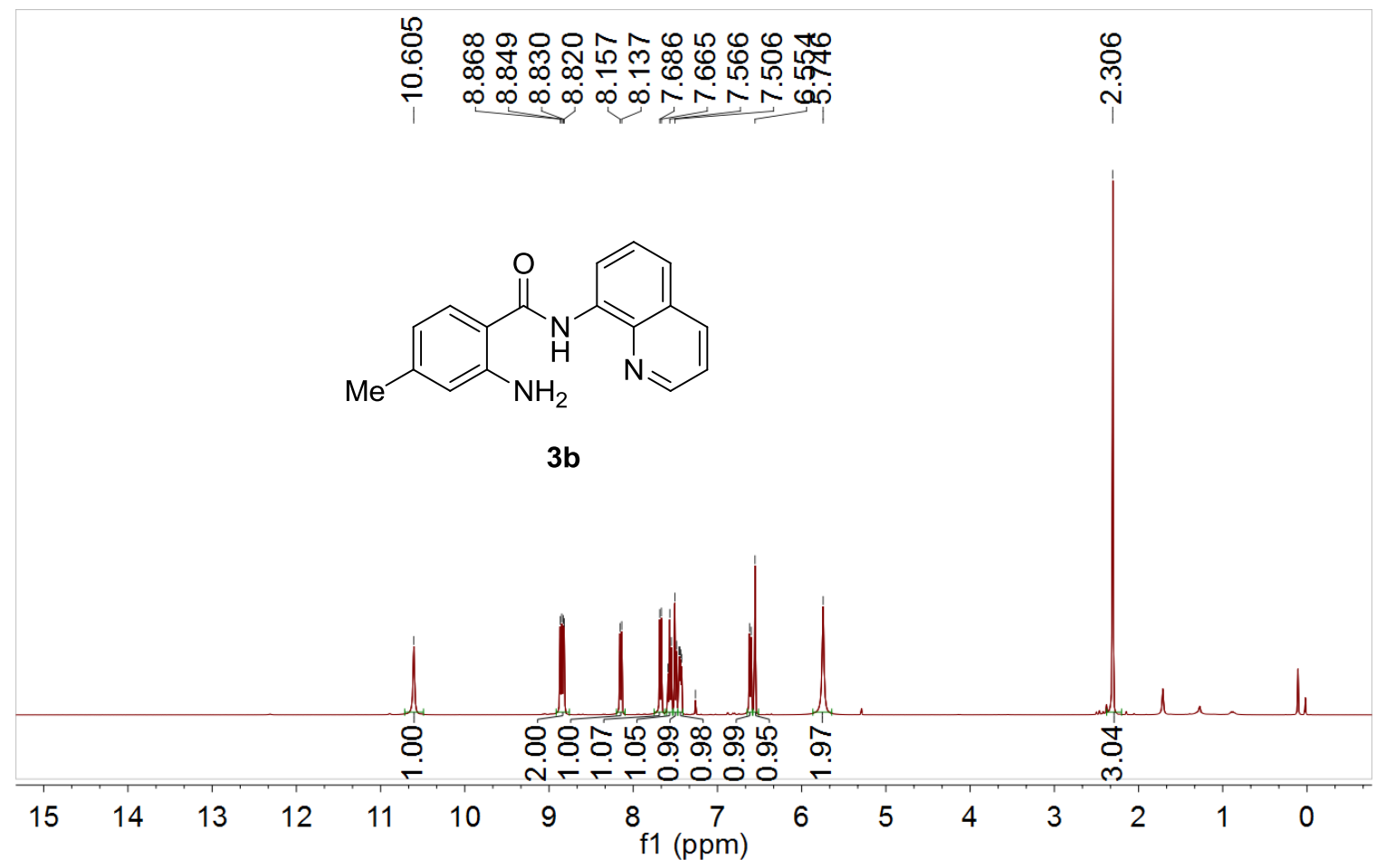

${ }^{13} \mathrm{C}$ NMR Spectrum of $\mathbf{3 b}$

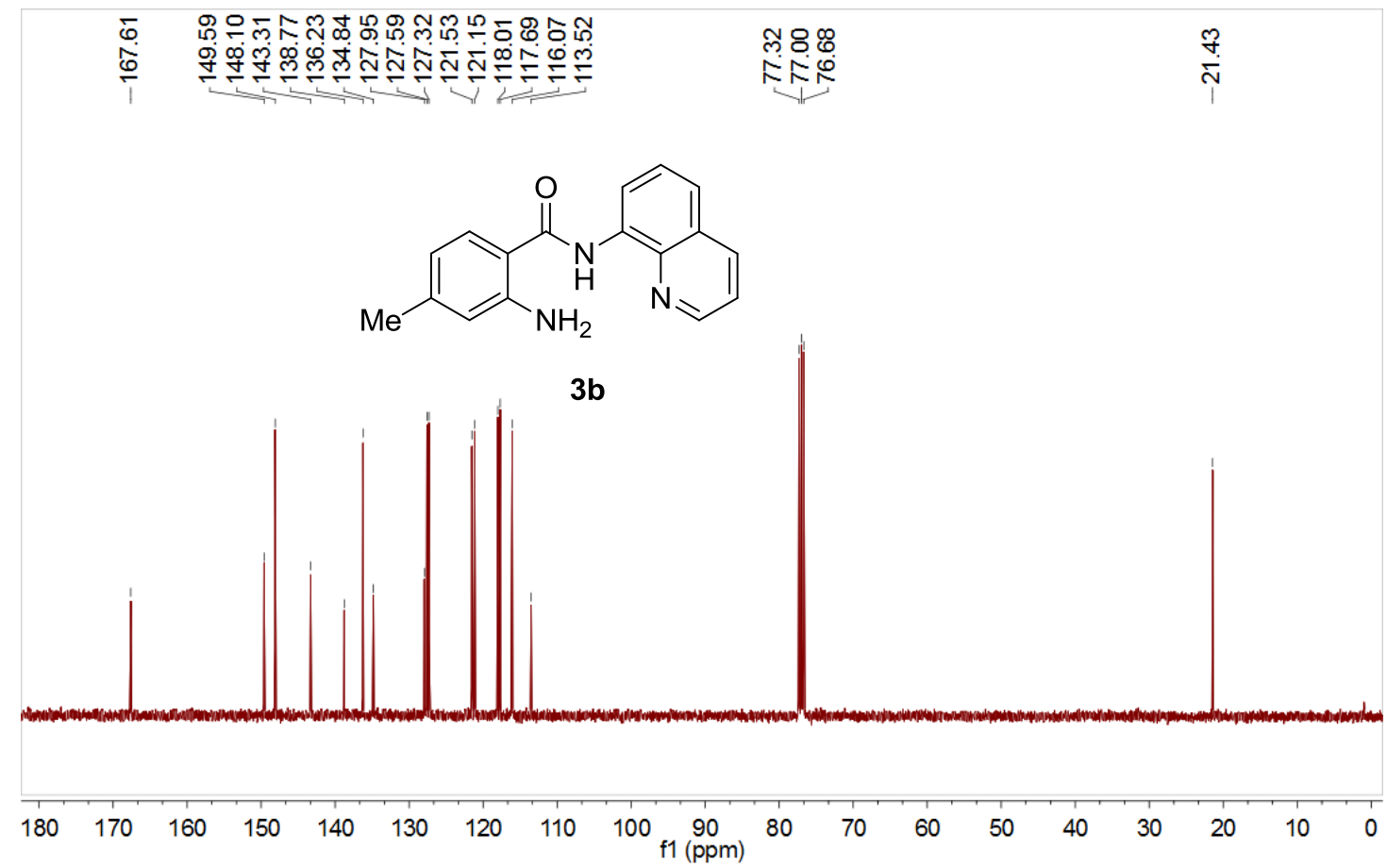


${ }^{1} \mathrm{H}$ NMR Spectrum of $\mathbf{3 c}$

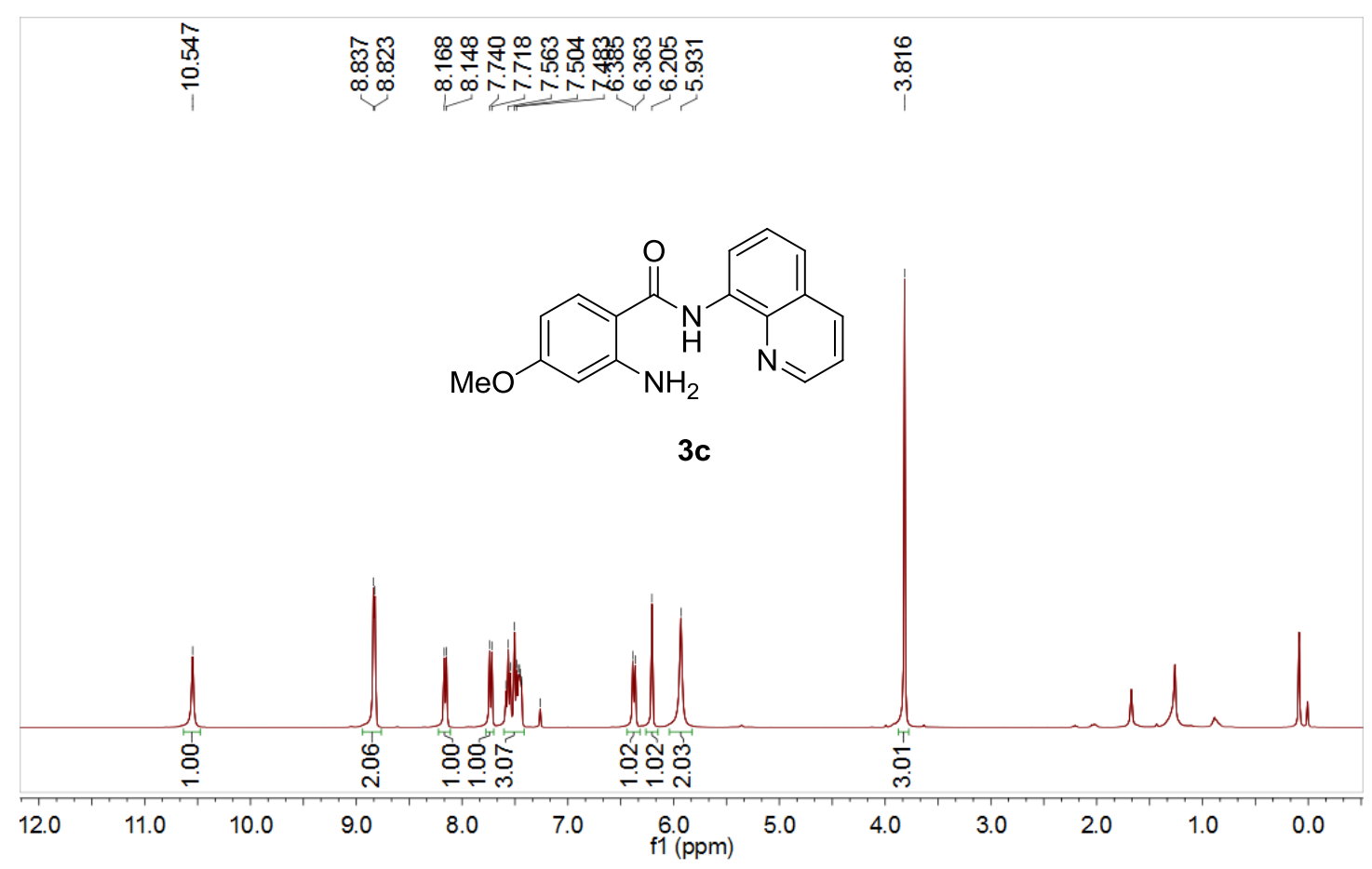

${ }^{13} \mathrm{C}$ NMR Spectrum of $\mathbf{3 c}$

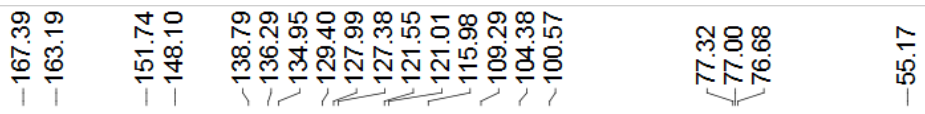

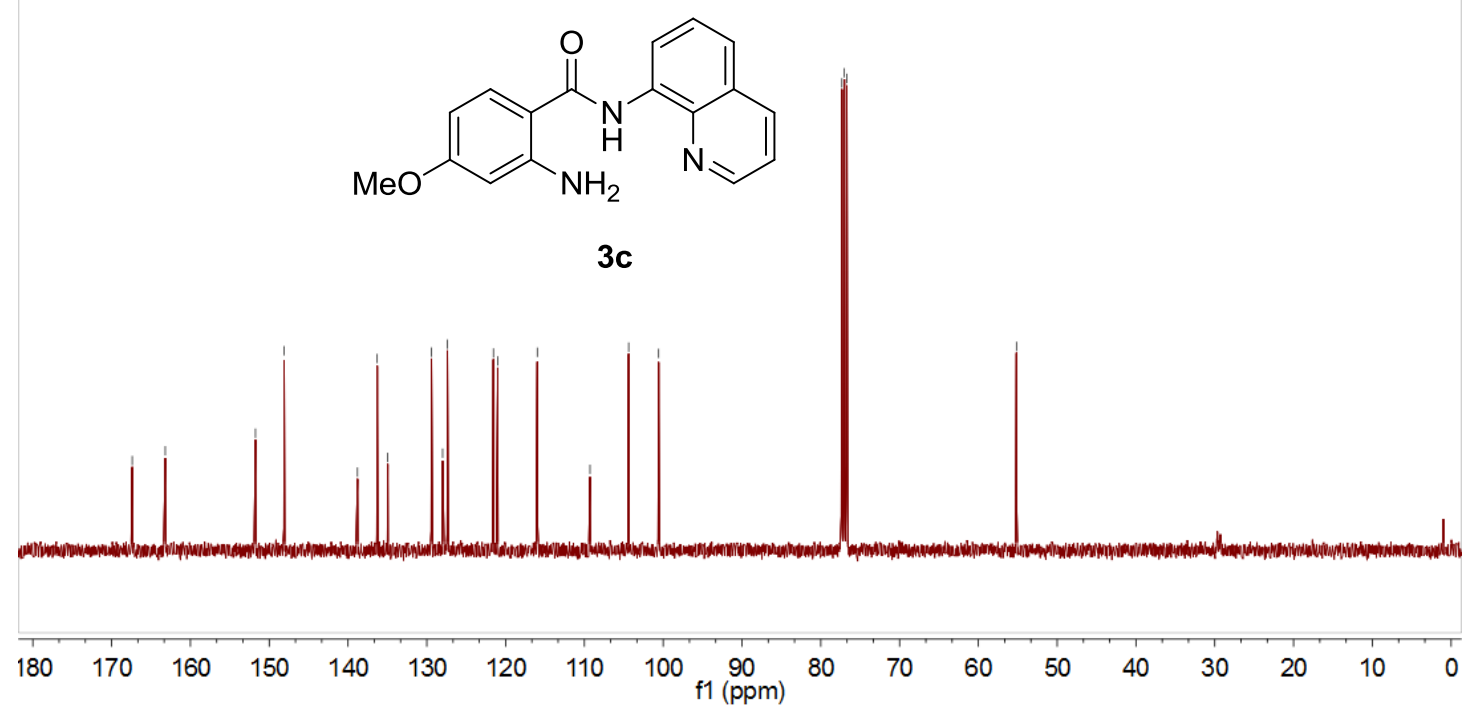


${ }^{1} \mathrm{H}$ NMR Spectrum of $\mathbf{3 d}$

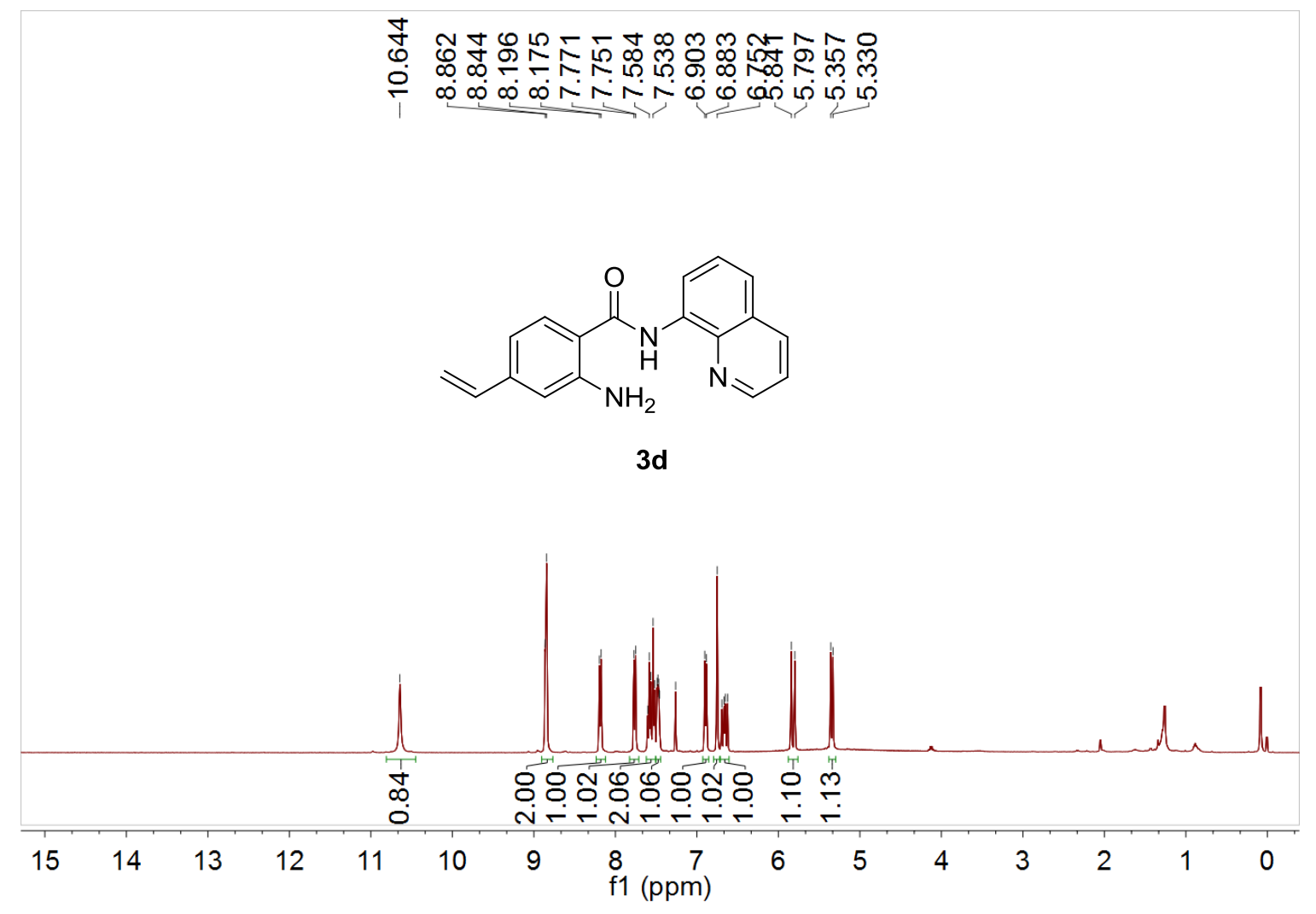

${ }^{13} \mathrm{C}$ NMR Spectrum of $\mathbf{3 d}$

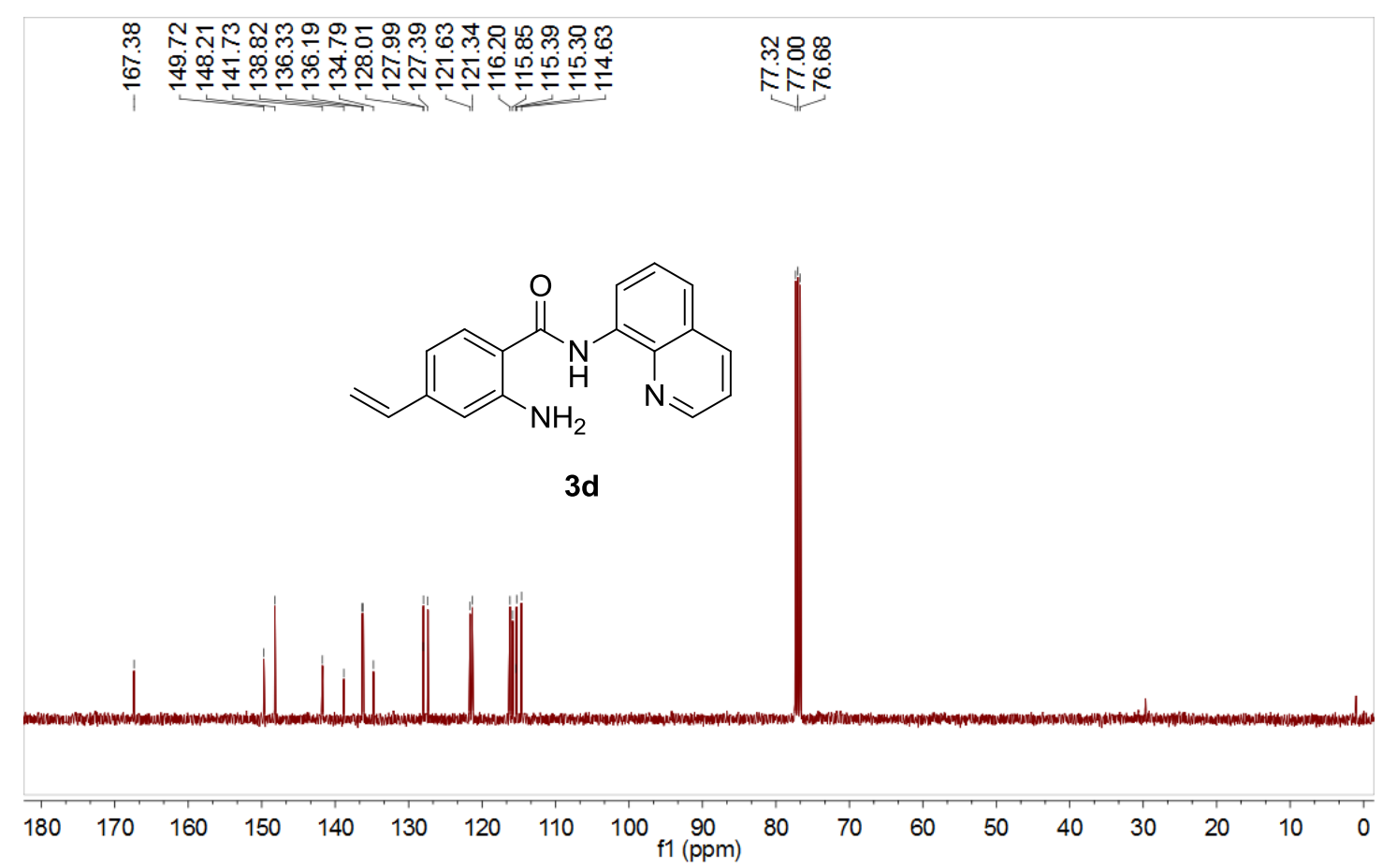


${ }^{1} \mathrm{H}$ NMR Spectrum of $\mathbf{3 e}$

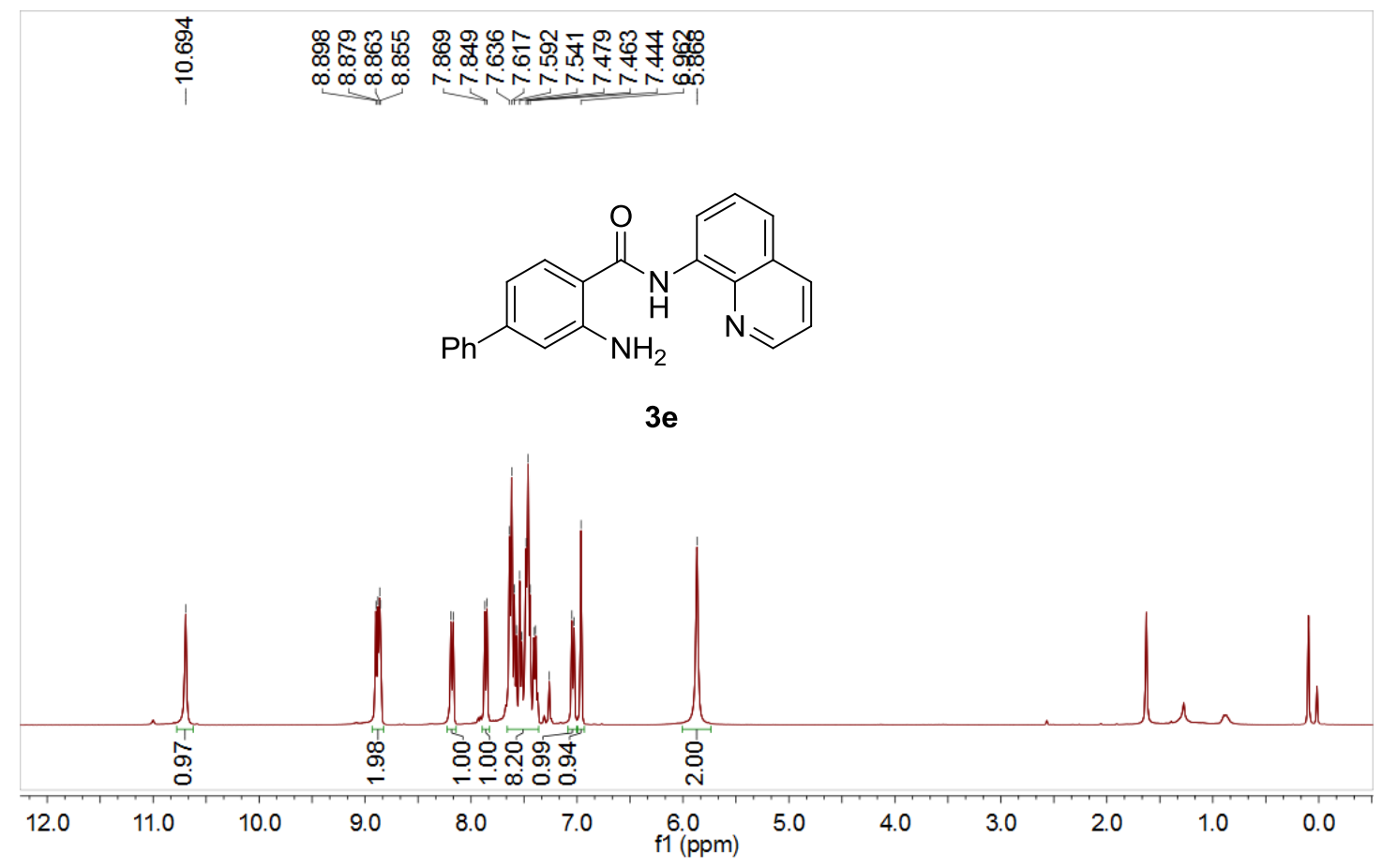

${ }^{13} \mathrm{C}$ NMR Spectrum of $\mathbf{3 e}$

ใ.

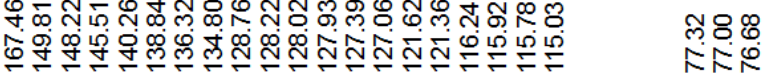

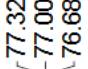

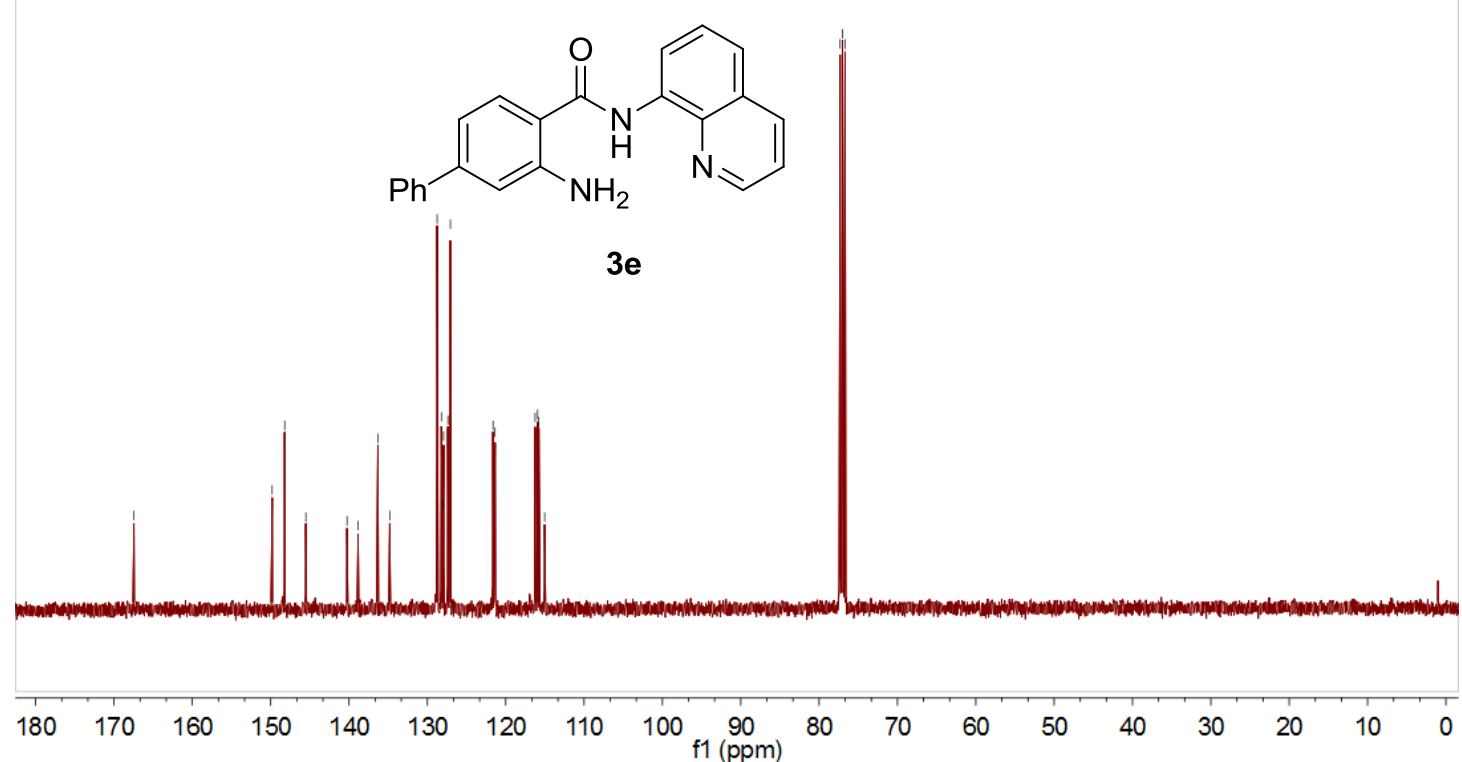


${ }^{1} \mathrm{H}$ NMR Spectrum of $\mathbf{3 f}$

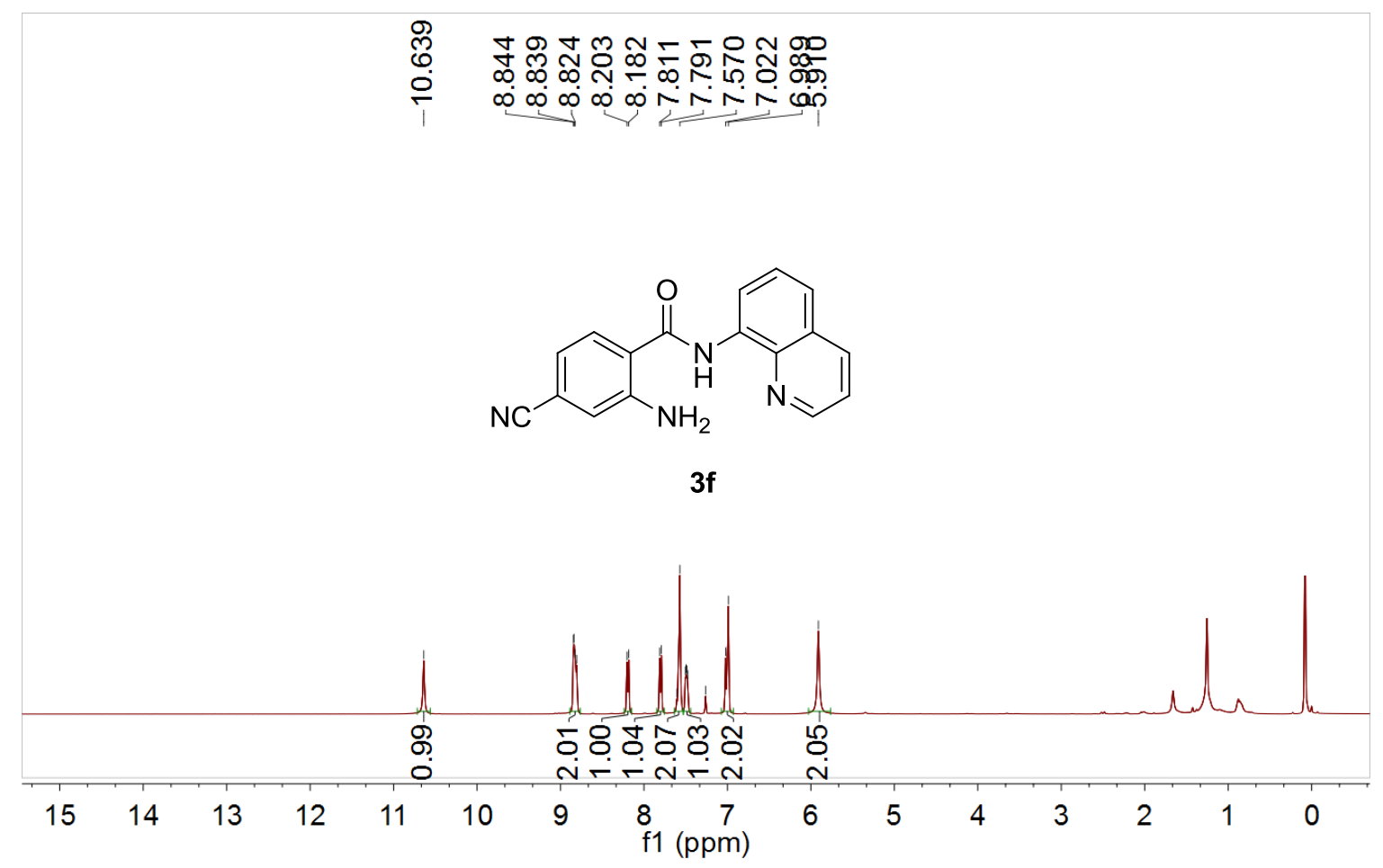

${ }^{13} \mathrm{C}$ NMR Spectrum of $\mathbf{3 f}$

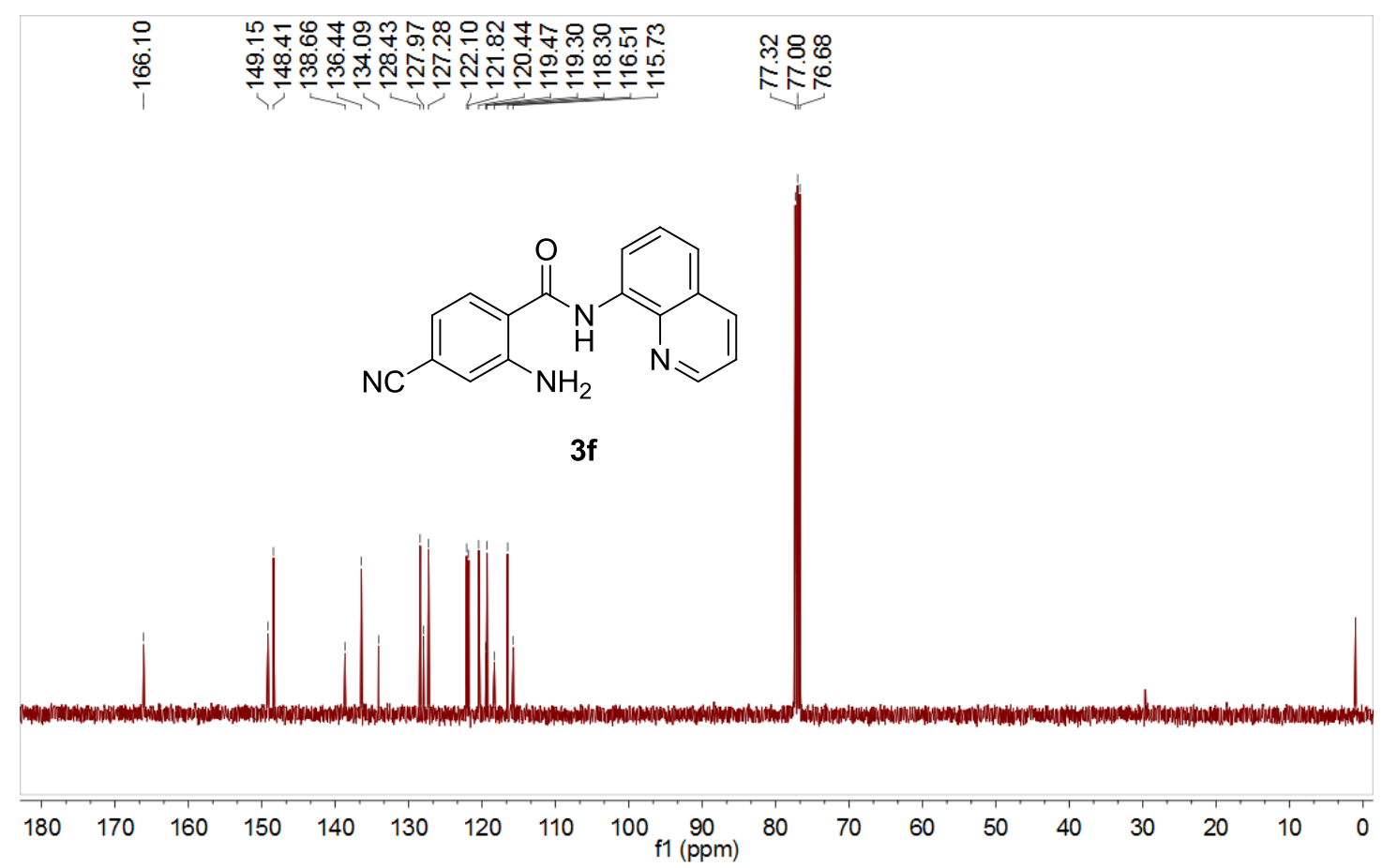


${ }^{1} \mathrm{H}$ NMR Spectrum of $\mathbf{3 g}$

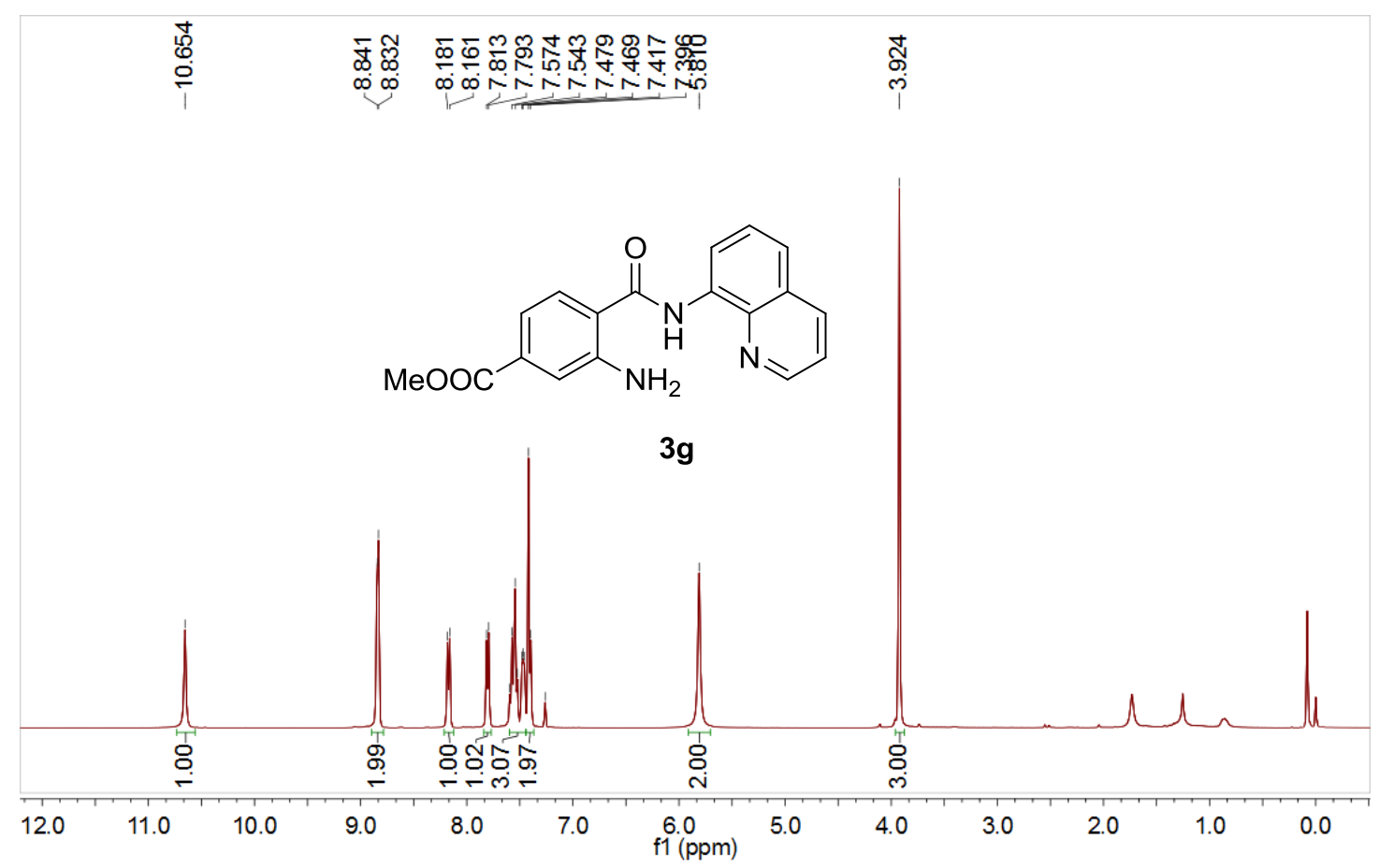

${ }^{13} \mathrm{C}$ NMR Spectrum of $\mathbf{3 g}$

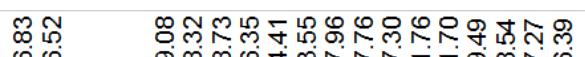

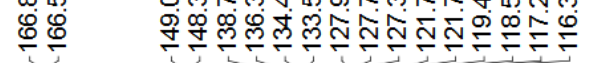

요ㅇㅛㅛ

స్ำ

MeOOC

$3 g$

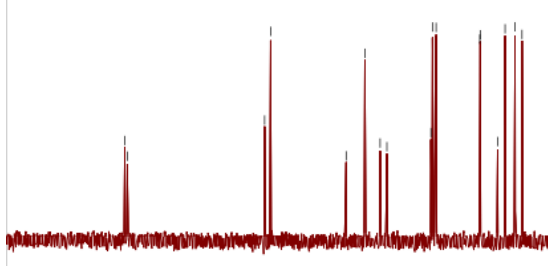

180

170160

150

140130

$120 \quad 110$

$100 \stackrel{90}{90}$

8070 
${ }^{1} \mathrm{H}$ NMR Spectrum of $\mathbf{3 h}$

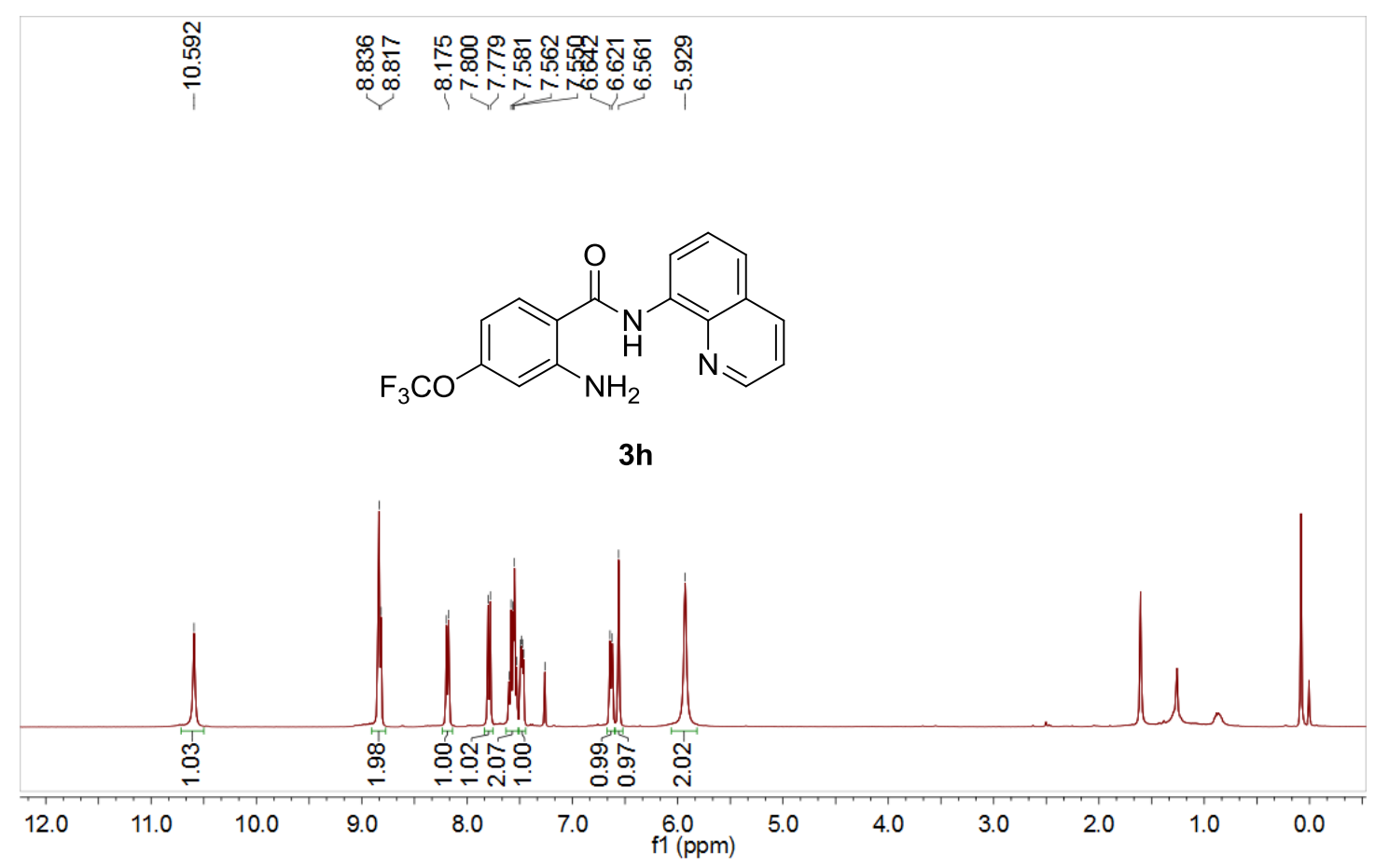

${ }^{13} \mathrm{C}$ NMR Spectrum of $\mathbf{3 h}$

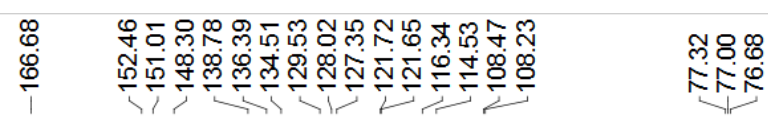

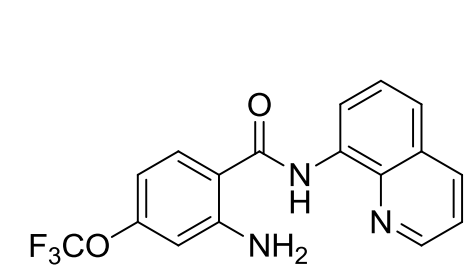

3h
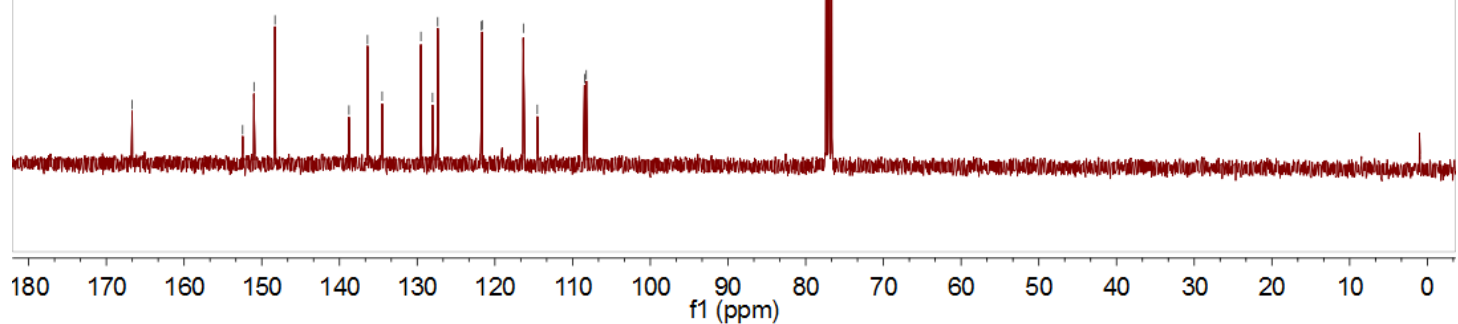
${ }^{19}$ F NMR Spectrum of $\mathbf{3 h}$

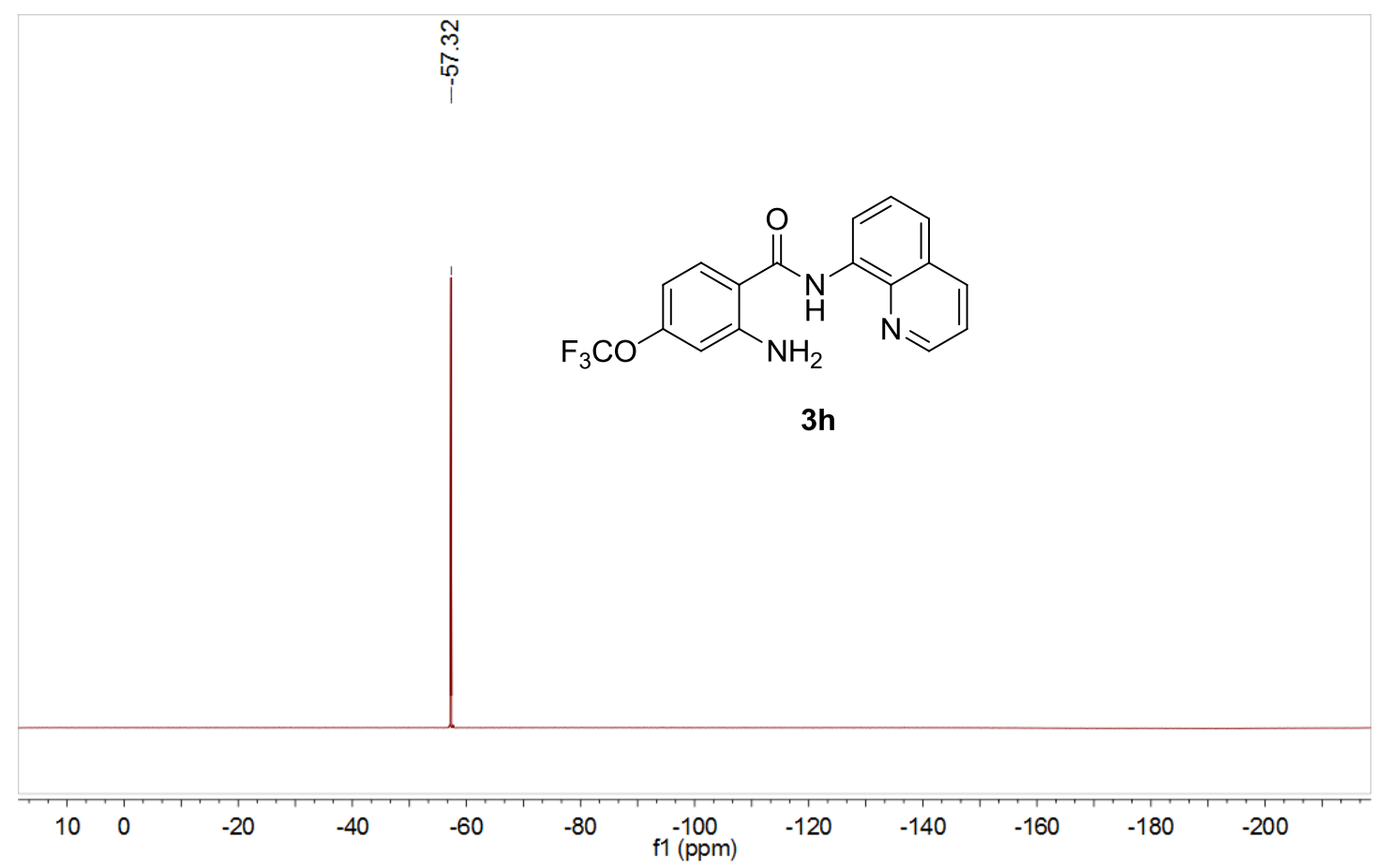

${ }^{1} \mathrm{H}$ NMR Spectrum of $\mathbf{3 i}$

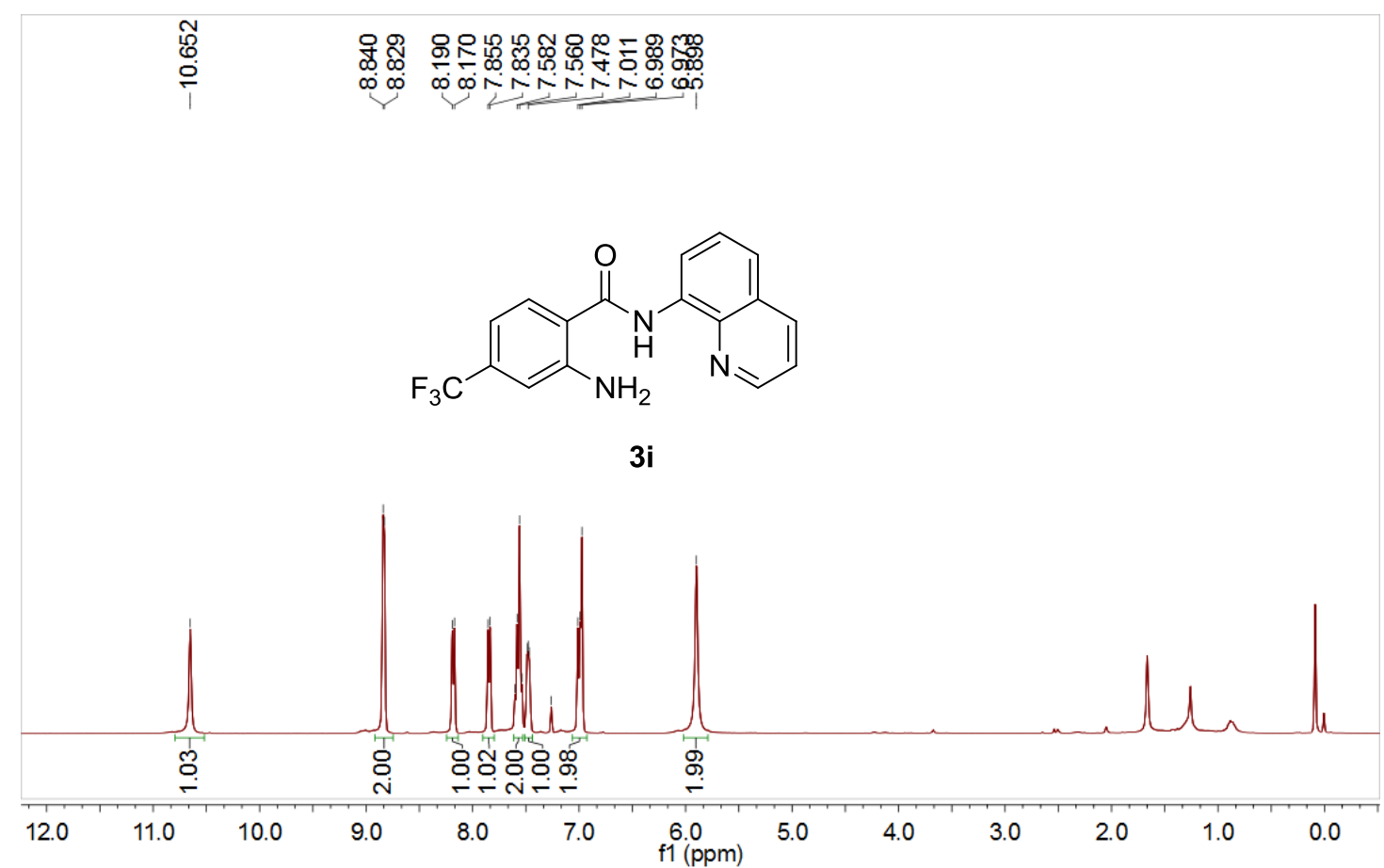


${ }^{13} \mathrm{C}$ NMR Spectrum of $\mathbf{3 i}$

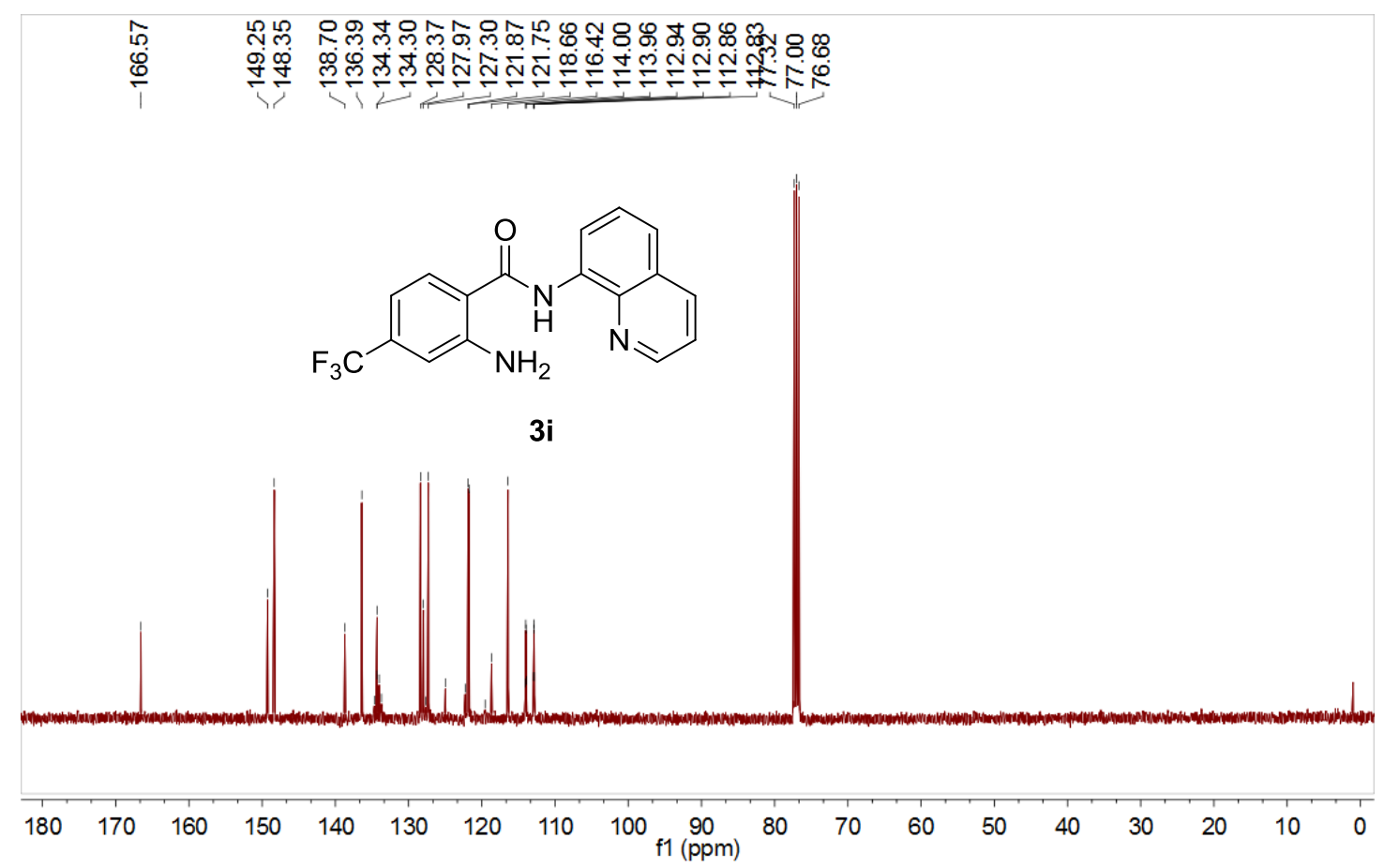

${ }^{19} \mathrm{~F}$ NMR Spectrum of $\mathbf{3 i}$

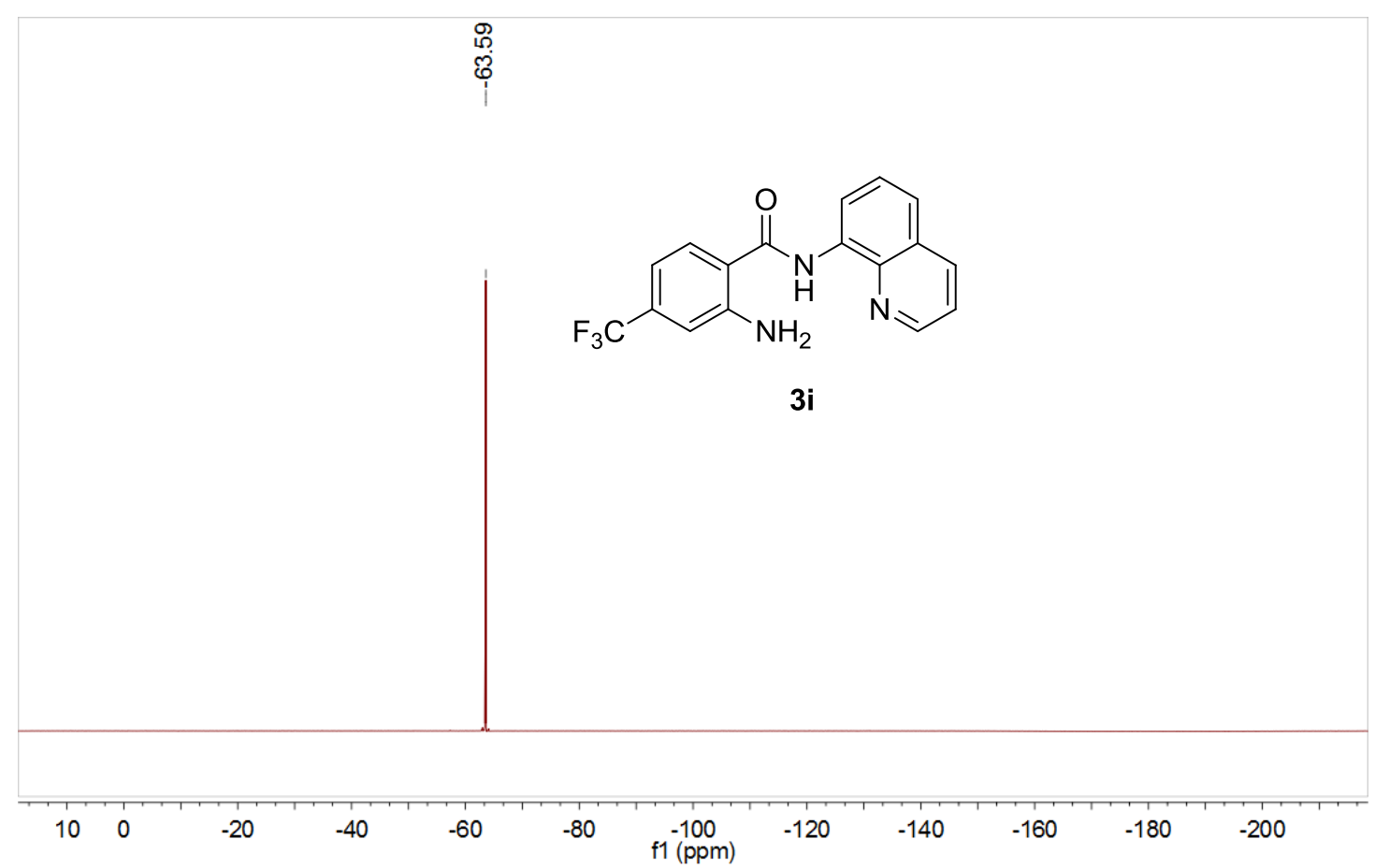


${ }^{1} \mathrm{H}$ NMR Spectrum of $\mathbf{3 j}$

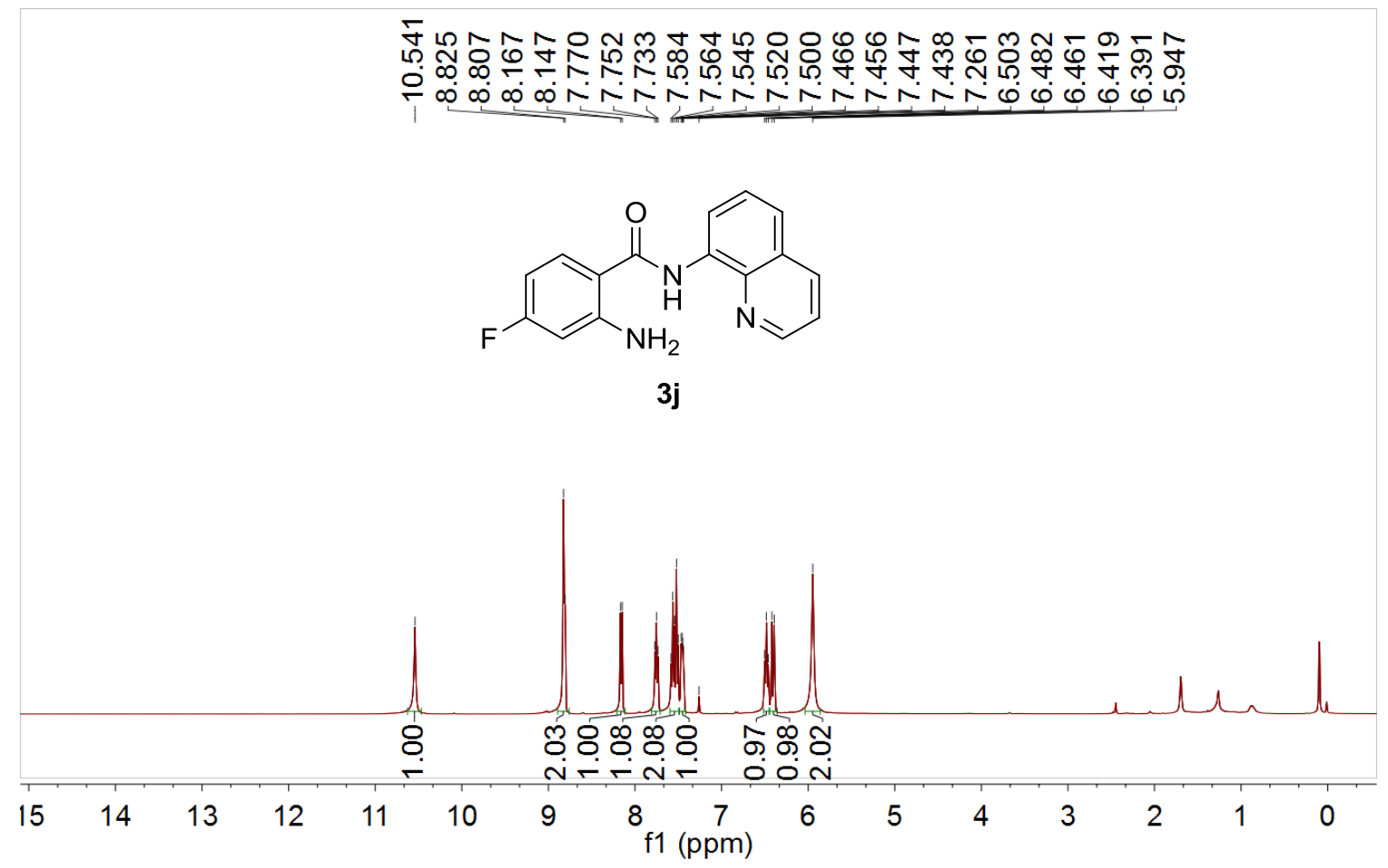

${ }^{13} \mathrm{C}$ NMR Spectrum of $\mathbf{3 j}$

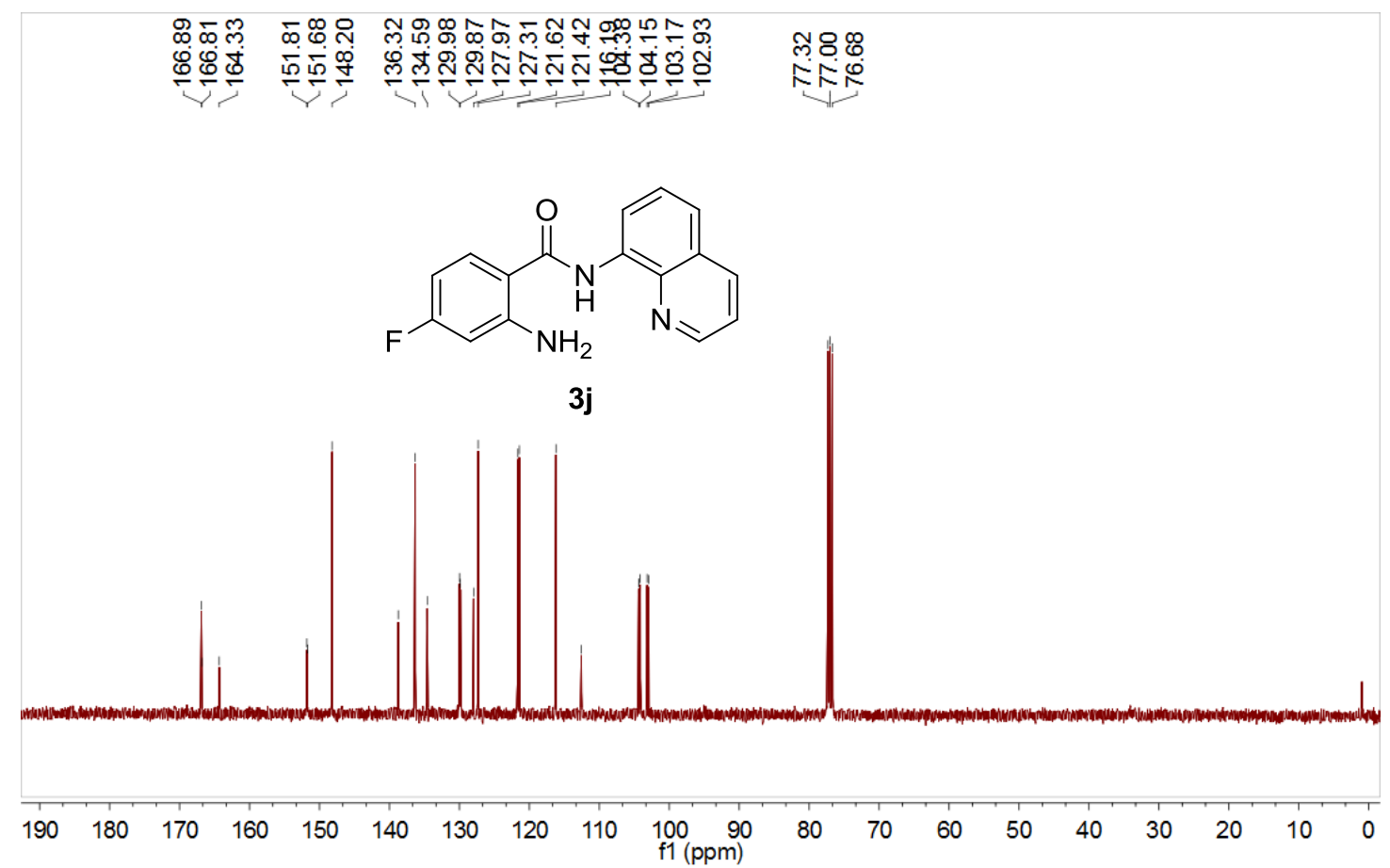




\section{${ }^{19} \mathrm{~F}$ NMR Spectrum of $\mathbf{3 j}$}

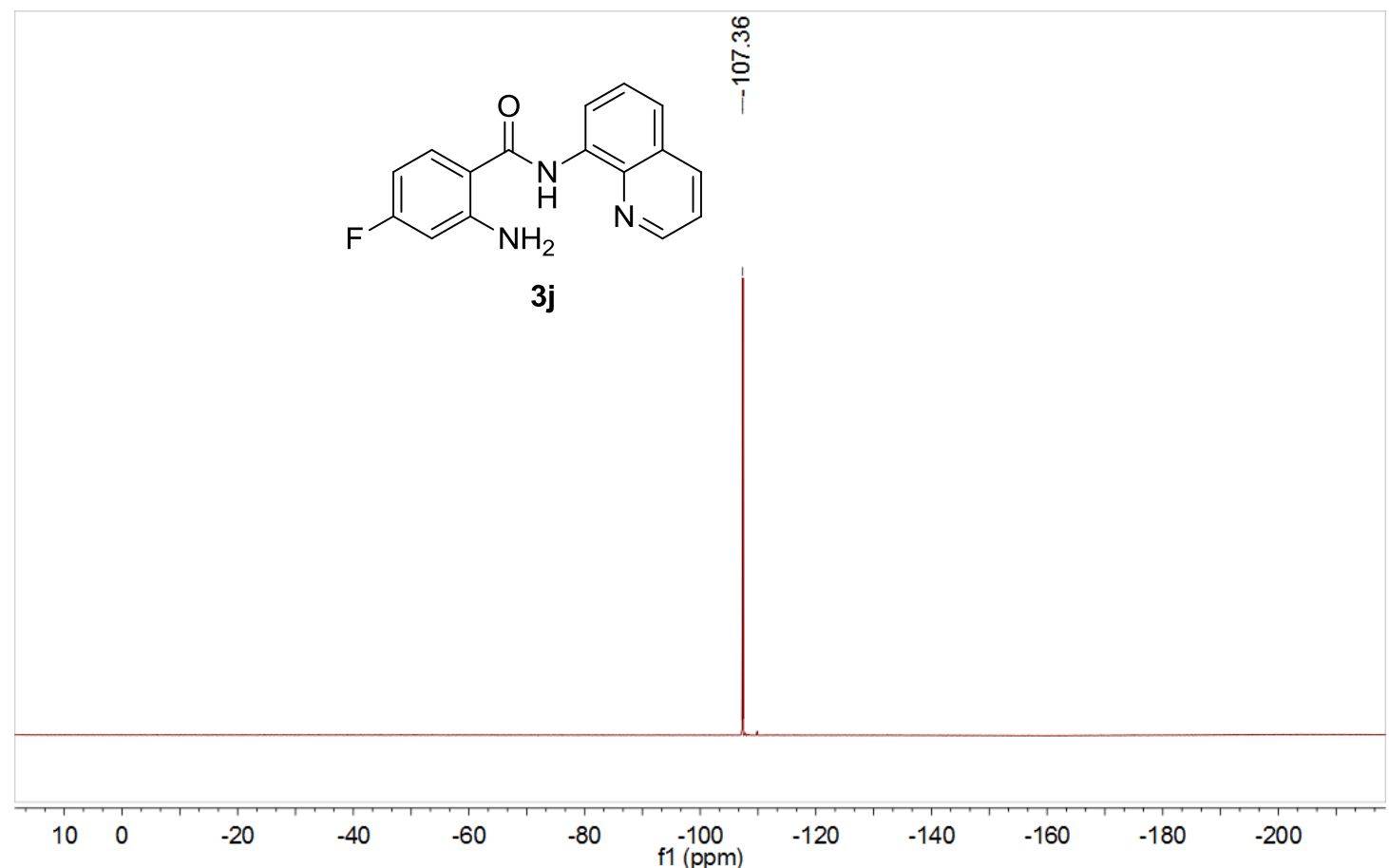

${ }^{1} \mathrm{H}$ NMR Spectrum of $\mathbf{3 k}$

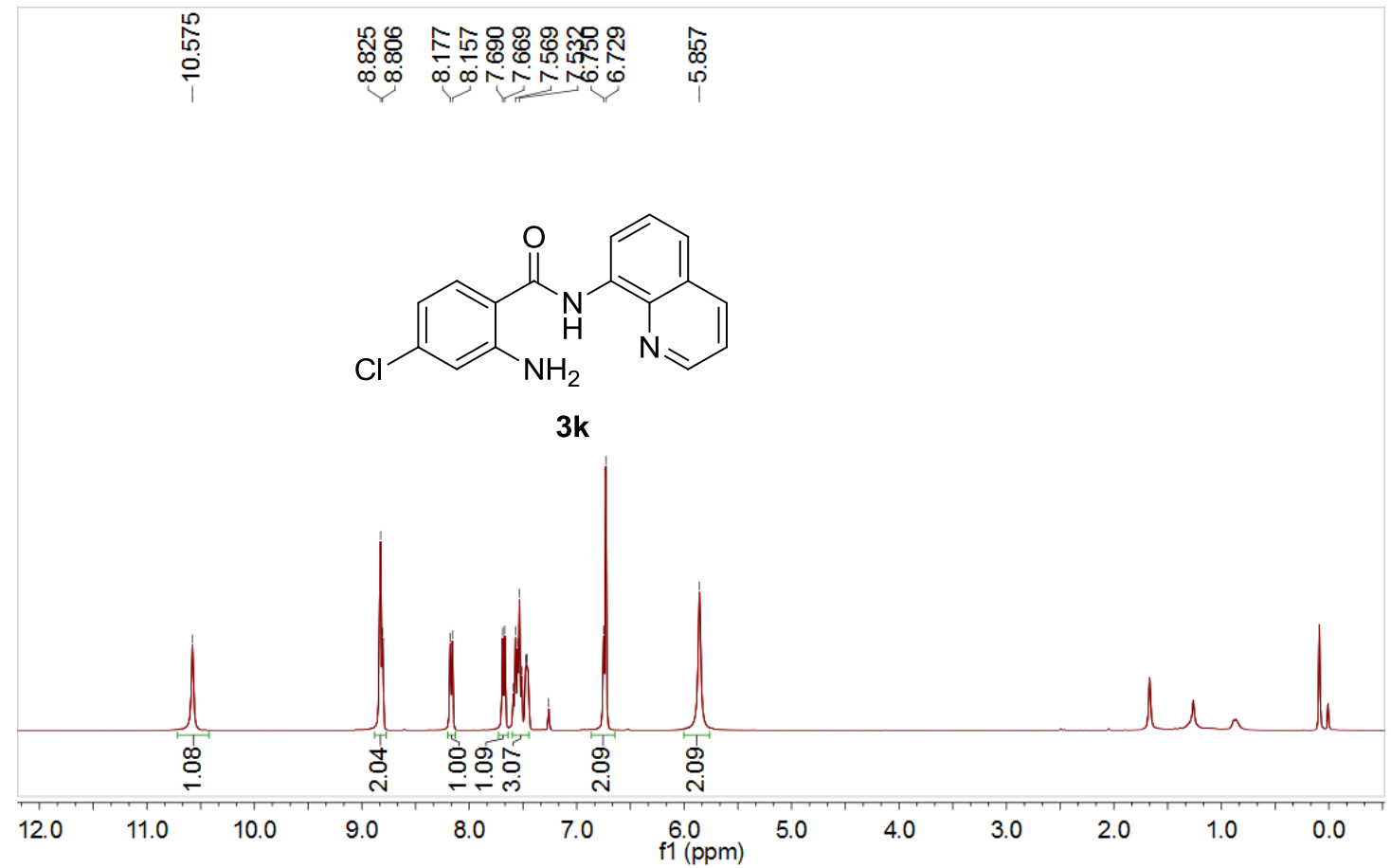


${ }^{13} \mathrm{C}$ NMR Spectrum of $\mathbf{3 k}$

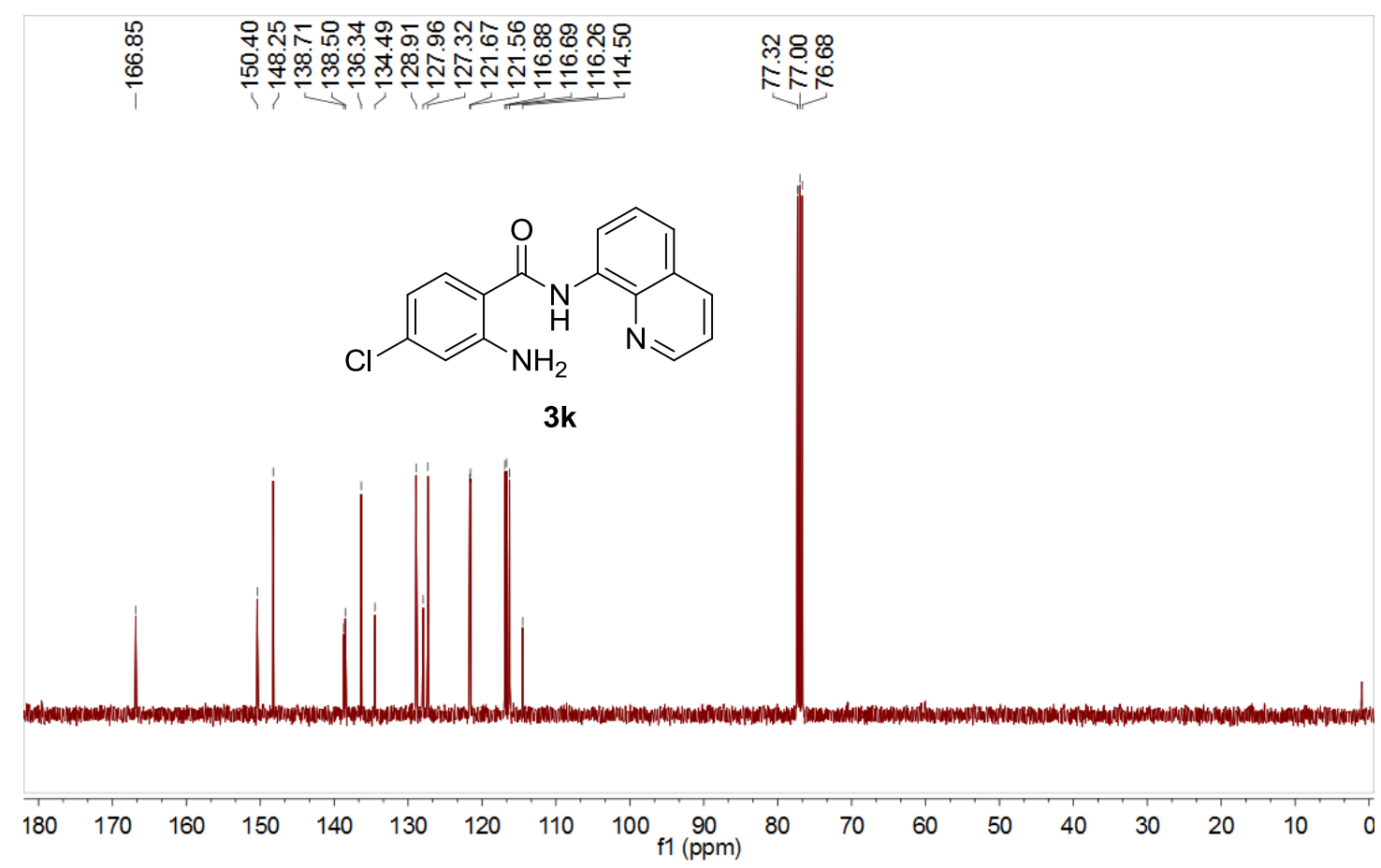

${ }^{1} \mathrm{H}$ NMR Spectrum of $\mathbf{3 l}$

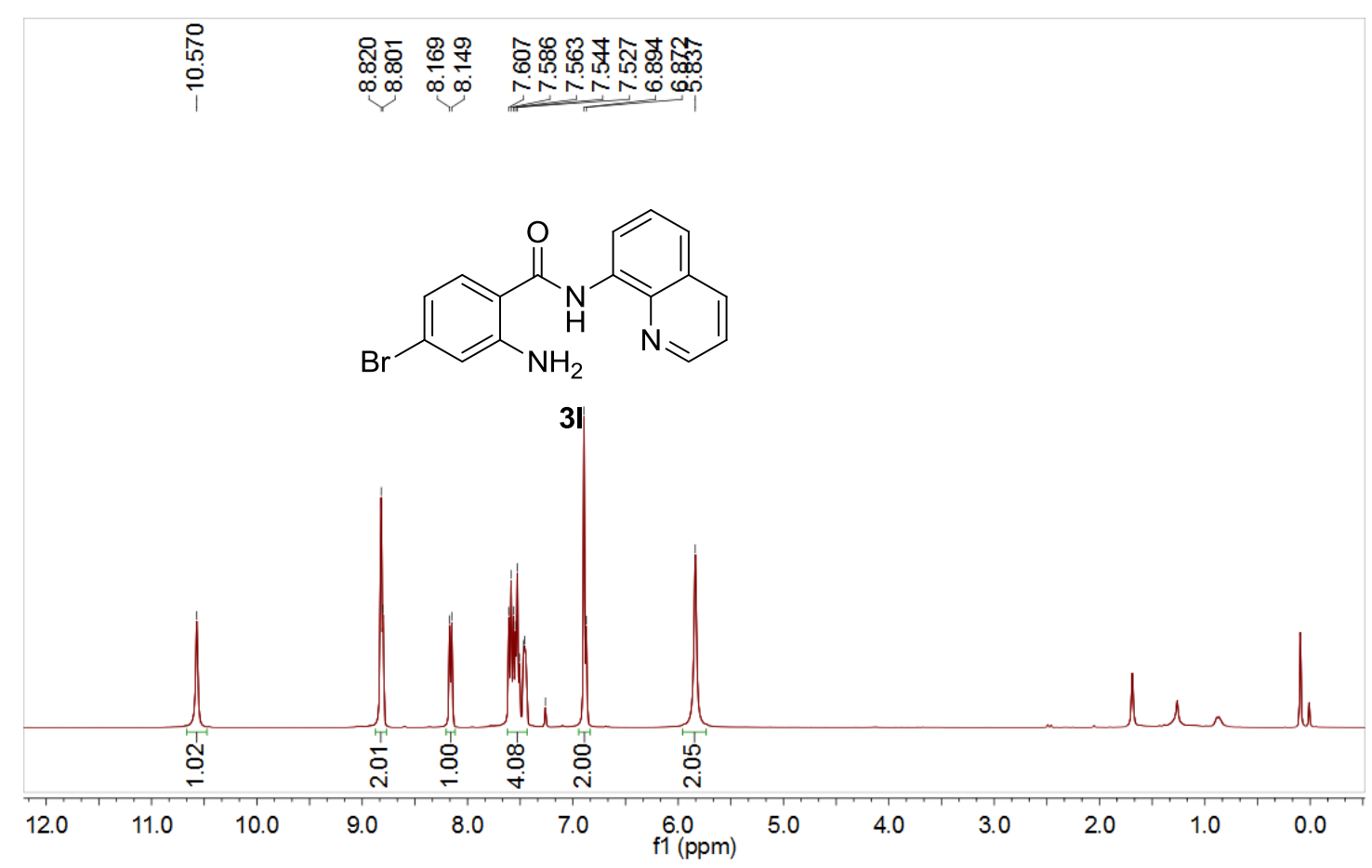


${ }^{13} \mathrm{C}$ NMR Spectrum of $\mathbf{3 I}$

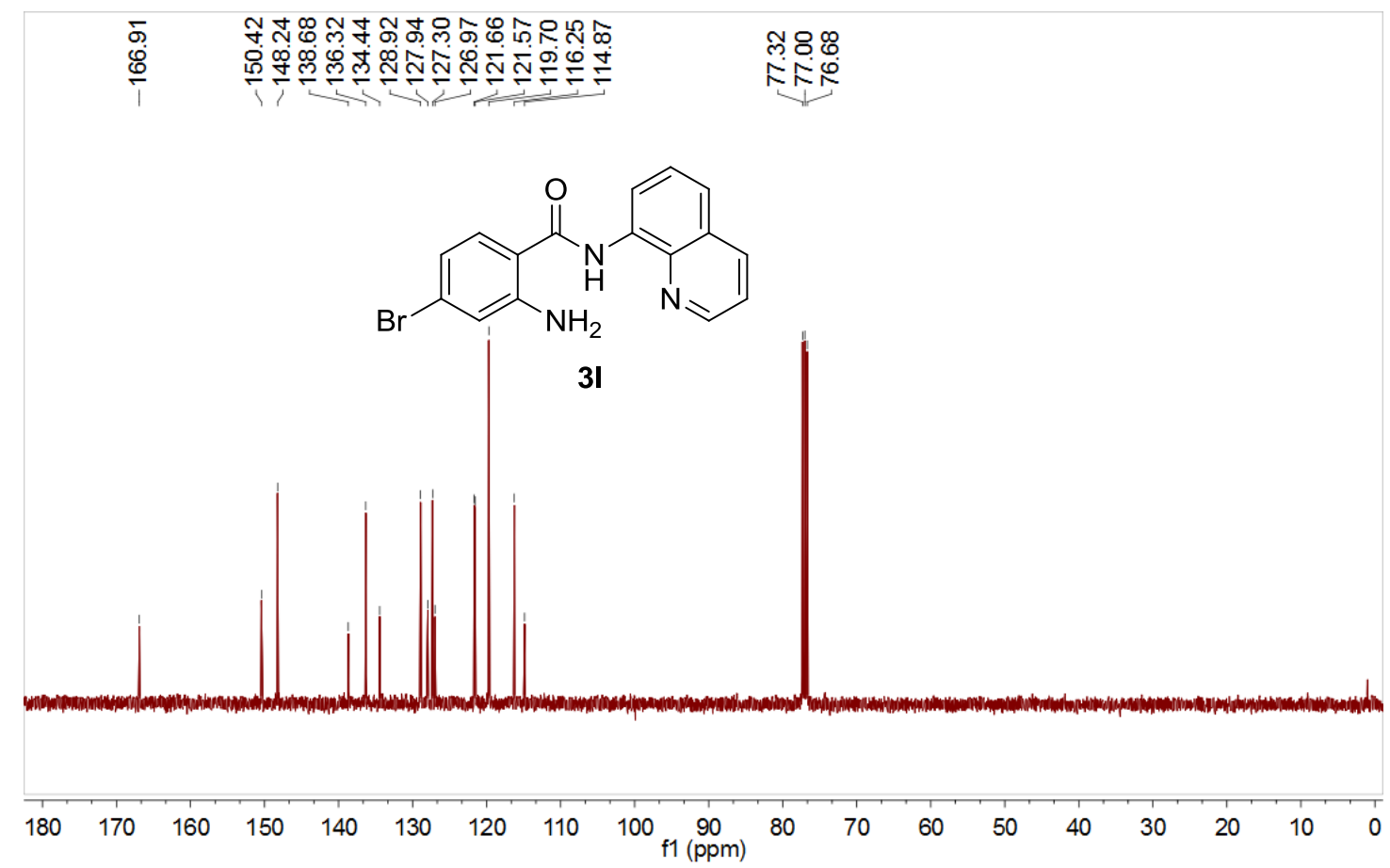

${ }^{1} \mathrm{H}$ NMR Spectrum of $\mathbf{3 m}$

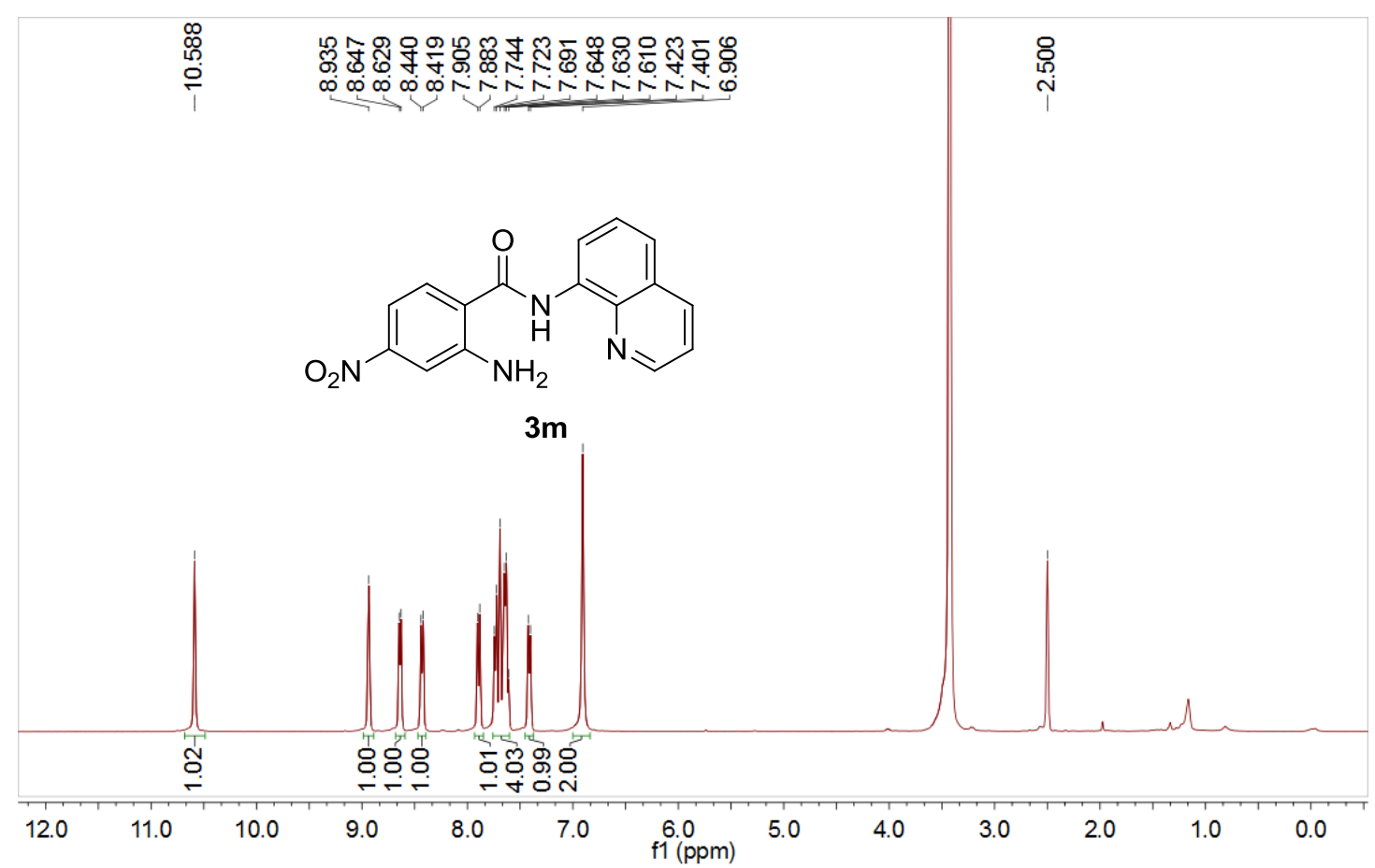


${ }^{13} \mathrm{C}$ NMR Spectrum of $\mathbf{3 m}$

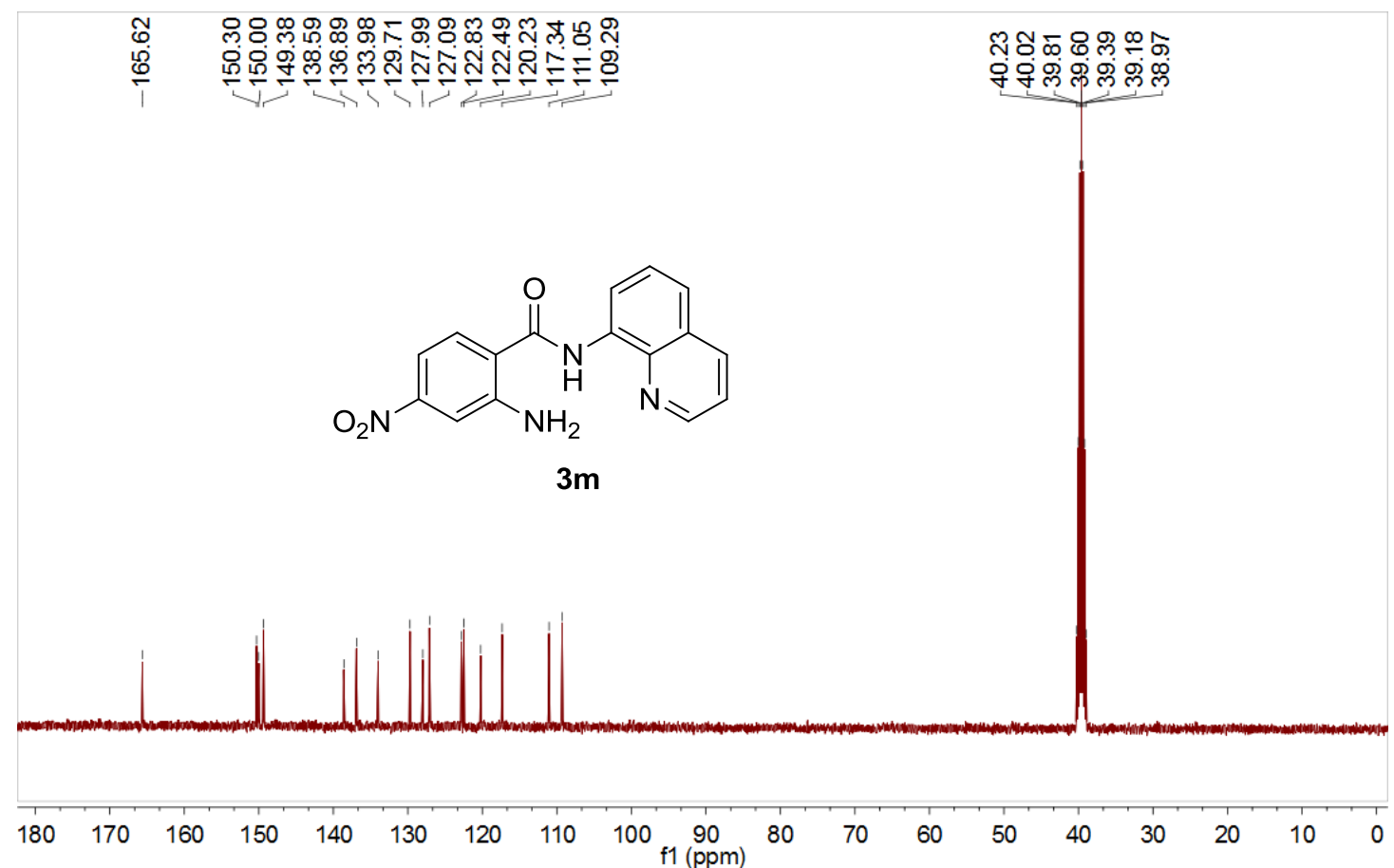

${ }^{1} \mathrm{H}$ NMR Spectrum of $\mathbf{3 n}$

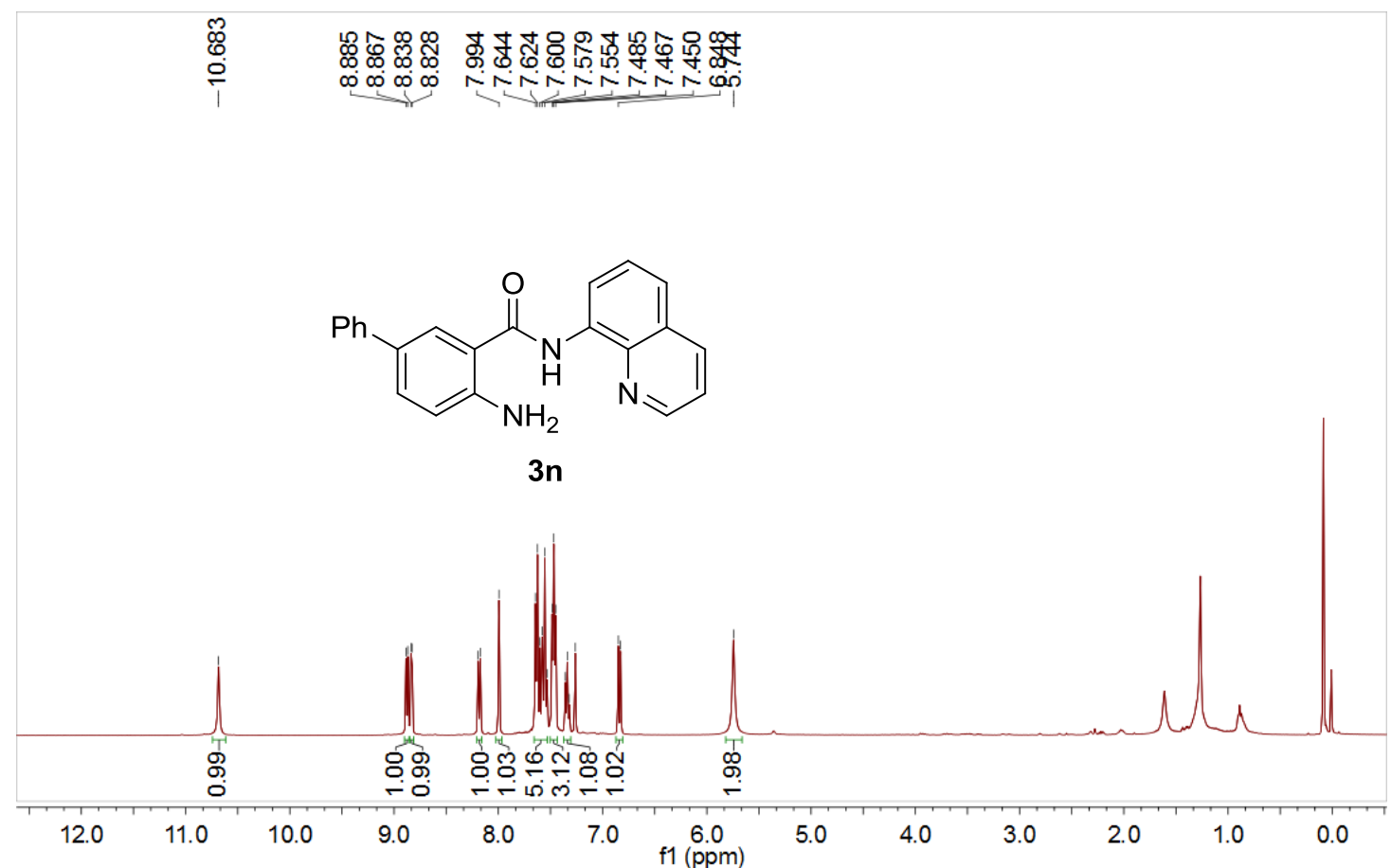


${ }^{13} \mathrm{C}$ NMR Spectrum of $\mathbf{3 n}$

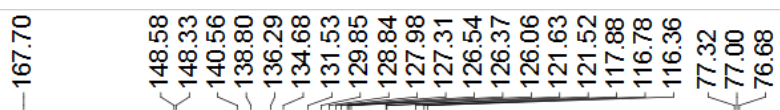

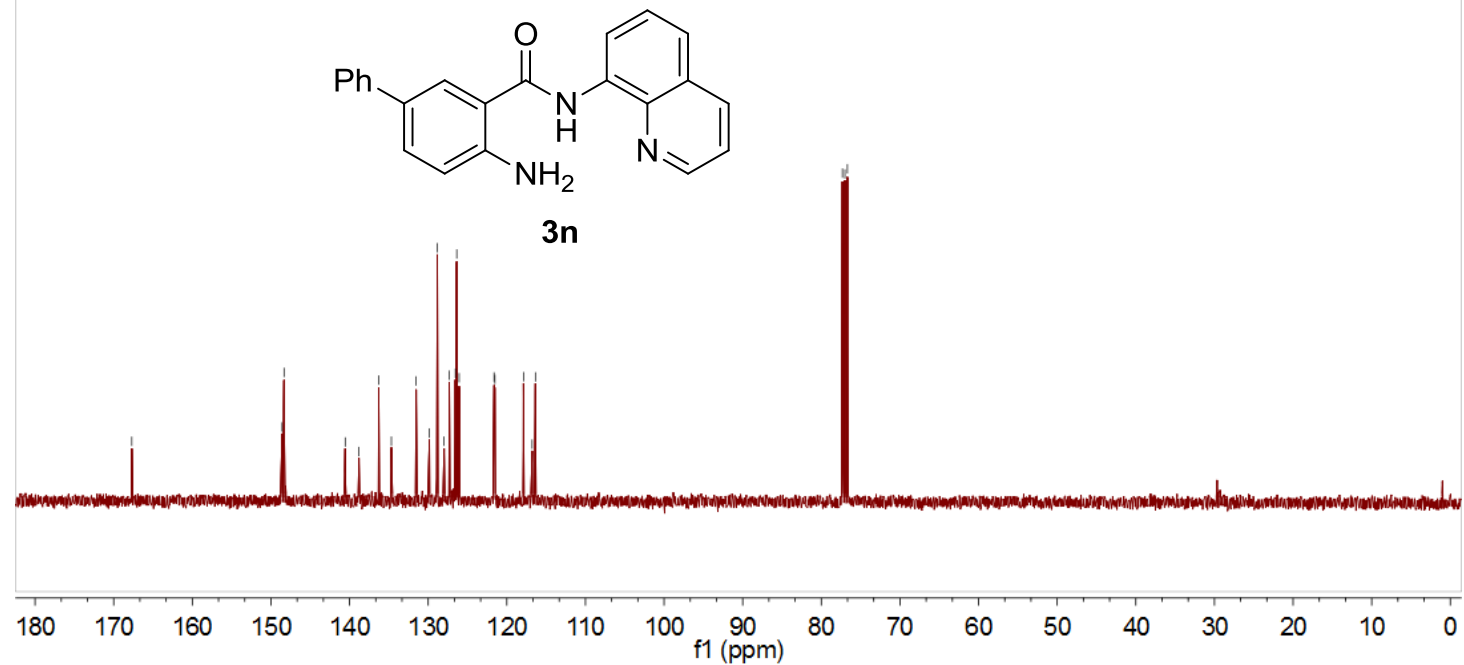

${ }^{1} \mathrm{H}$ NMR Spectrum of $\mathbf{3 o}$

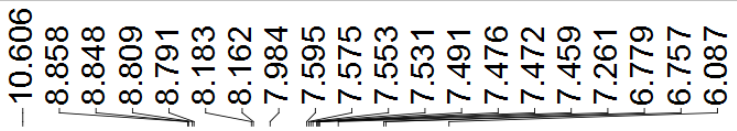

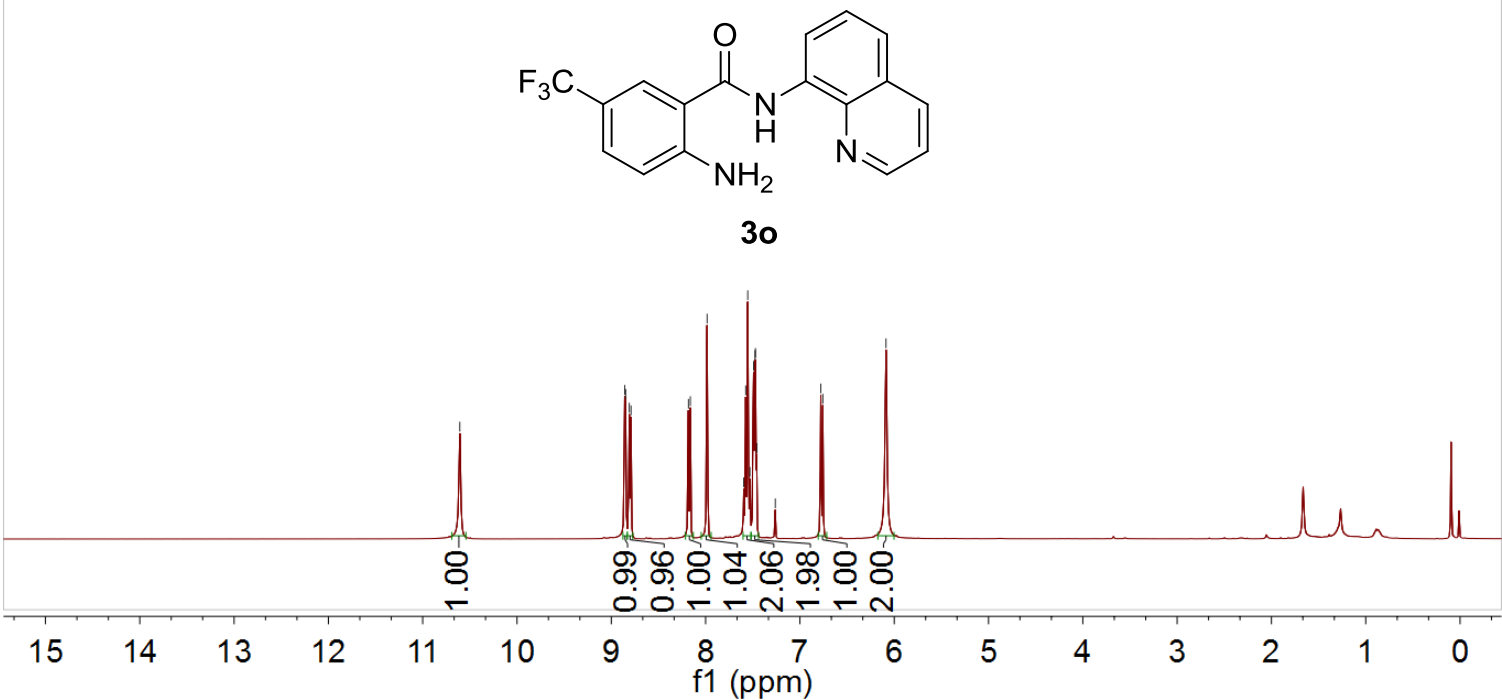


${ }^{13} \mathrm{C}$ NMR Spectrum of $\mathbf{3 o}$

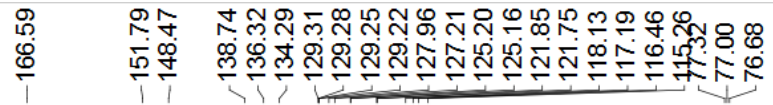<smiles>Nc1ccc(C(F)(F)F)cc1C(=O)Nc1cccc2cccnc12</smiles>

30

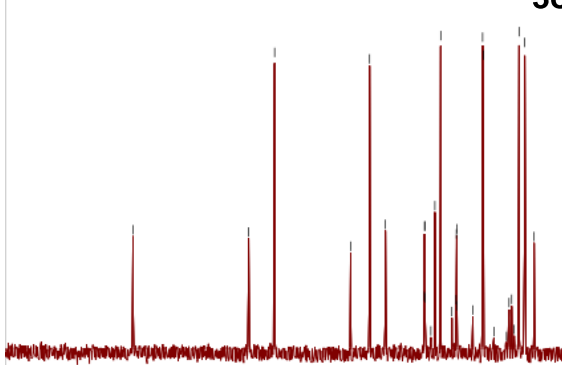

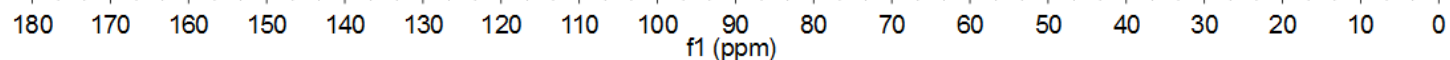

${ }^{19}$ F NMR Spectrum of 30

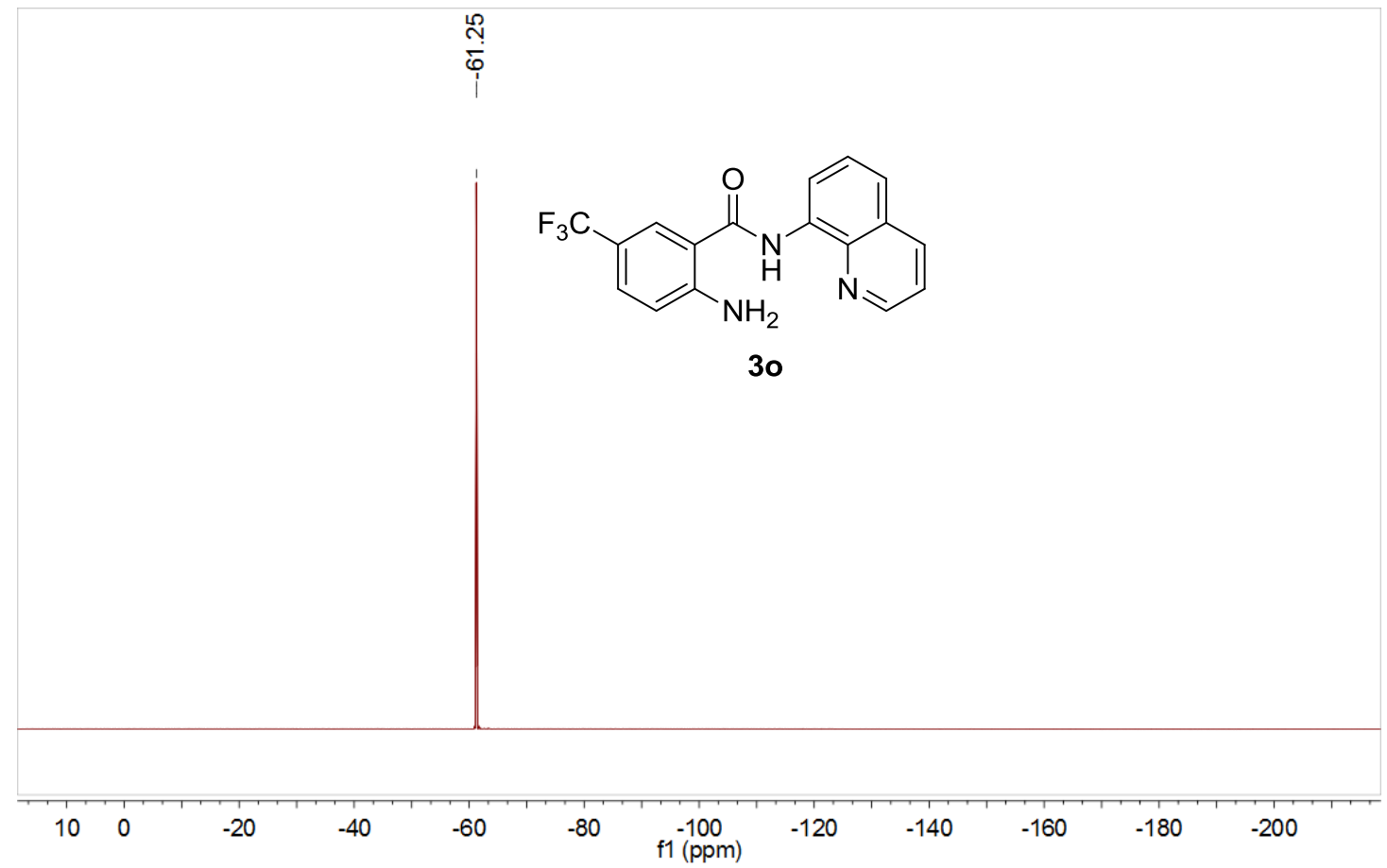


${ }^{1} \mathrm{H}$ NMR Spectrum of $\mathbf{3 p}$

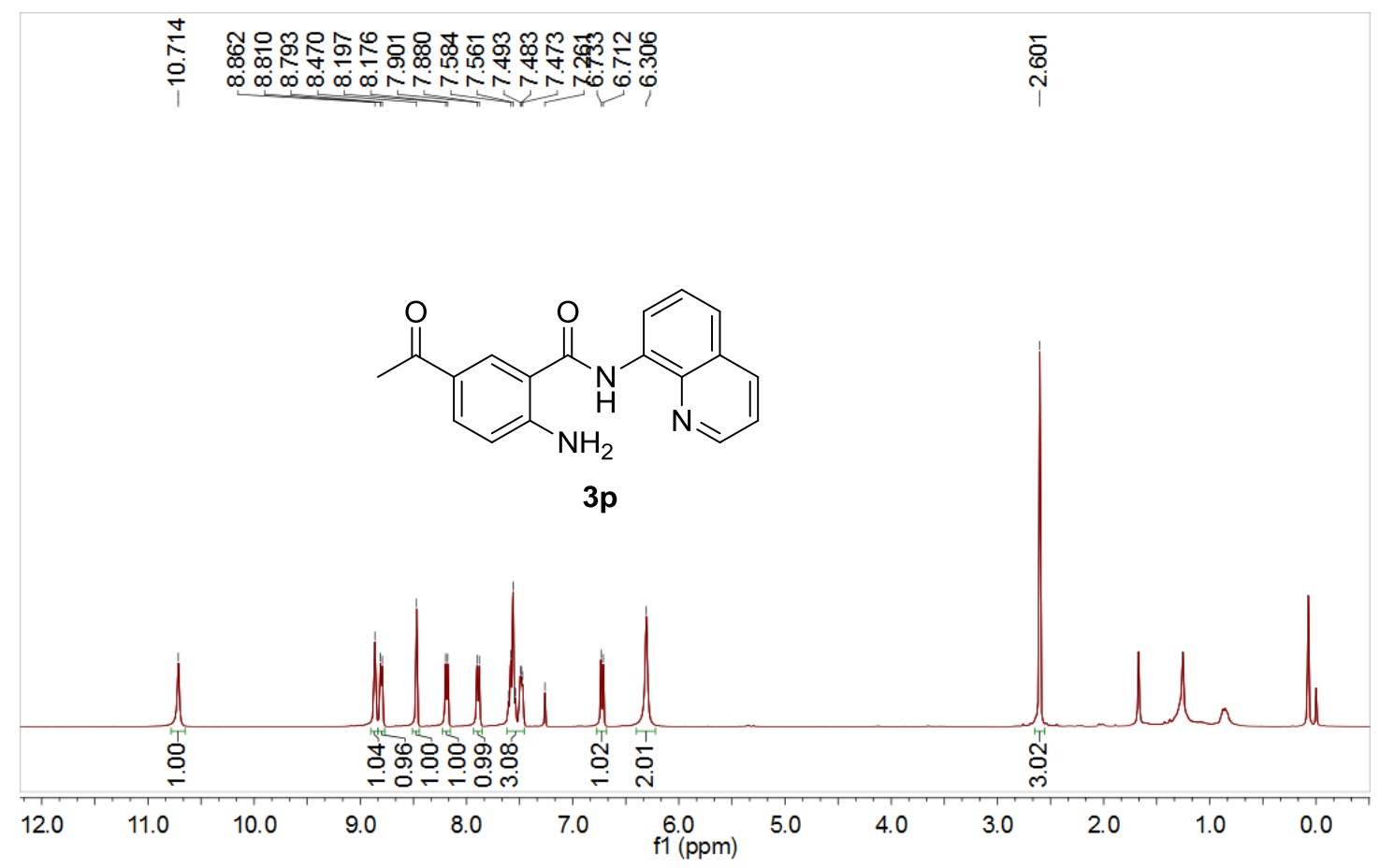

${ }^{13} \mathrm{C}$ NMR Spectrum of $\mathbf{3 p}$

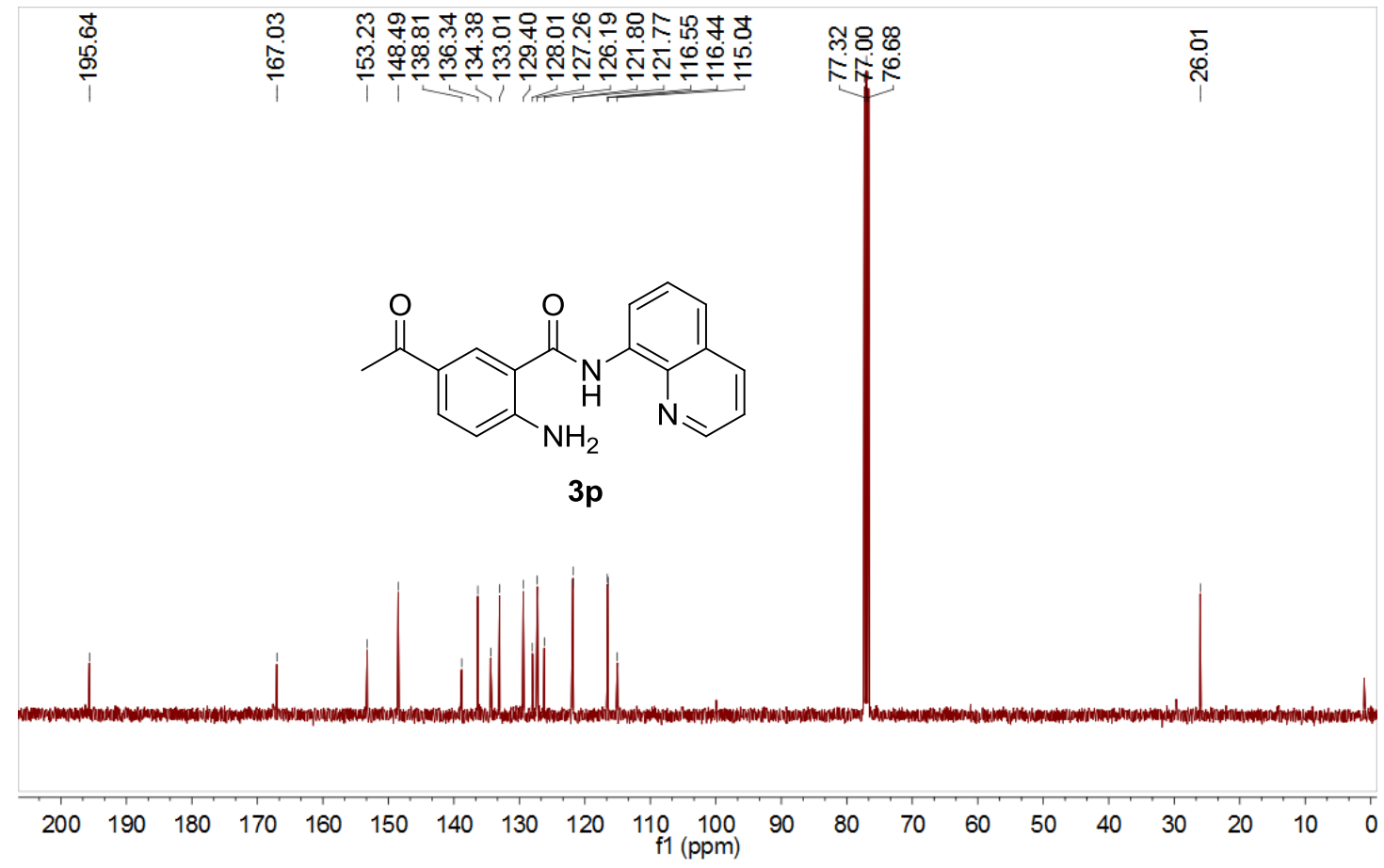


${ }^{1} \mathrm{H}$ NMR Spectrum of $\mathbf{3 q}$

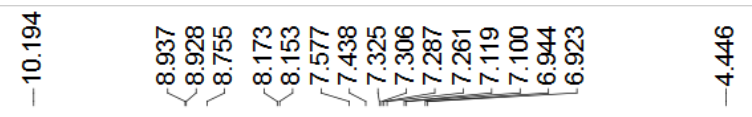<smiles>Nc1cccc(C(F)(F)F)c1C(=O)Nc1cccc2cccnc12</smiles>

$3 q$

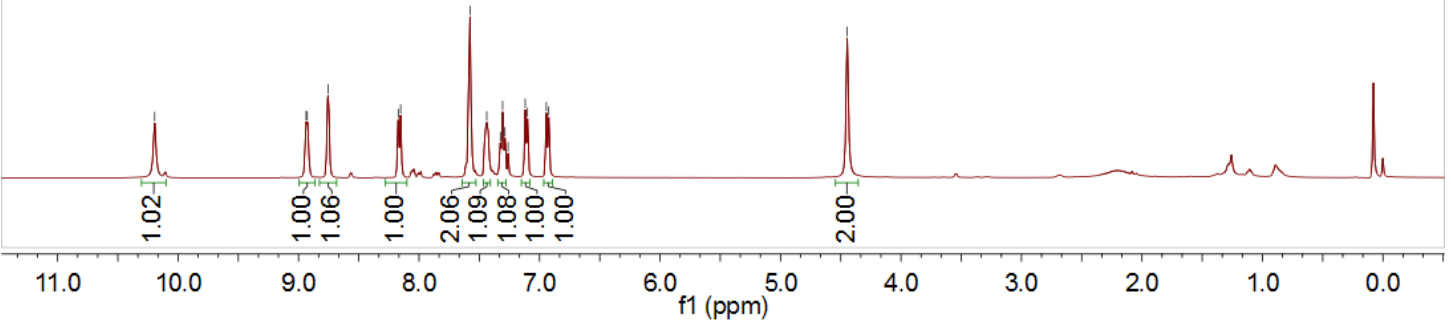

${ }^{13} \mathrm{C}$ NMR Spectrum of $\mathbf{3 q}$

$\begin{array}{ll}1 & 1 \\ 0 & 0\end{array}$<smiles>Nc1cccc(C(F)(F)F)c1C(=O)Nc1cccc2cccnc12</smiles>

$3 q$

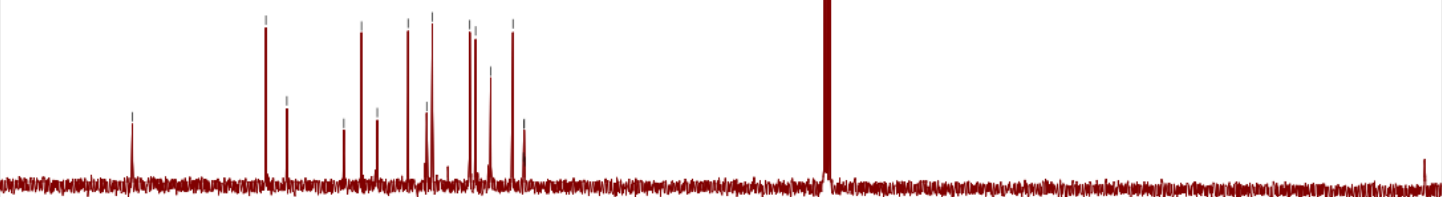




\section{${ }^{19}$ F NMR Spectrum of $\mathbf{3 q}$}

$\stackrel{\substack{\infty \\ \infty}}{\substack{p \\ i}}$<smiles>Nc1cccc(C(F)(F)F)c1C(=O)Nc1cccc2cccnc12</smiles>

$3 q$

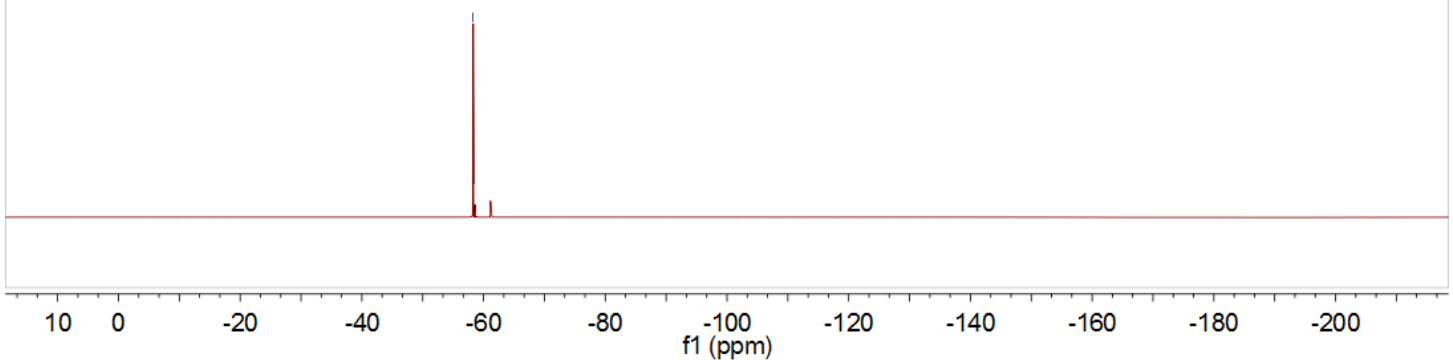

${ }^{1} \mathrm{H}$ NMR Spectrum of $\mathbf{3 r}$

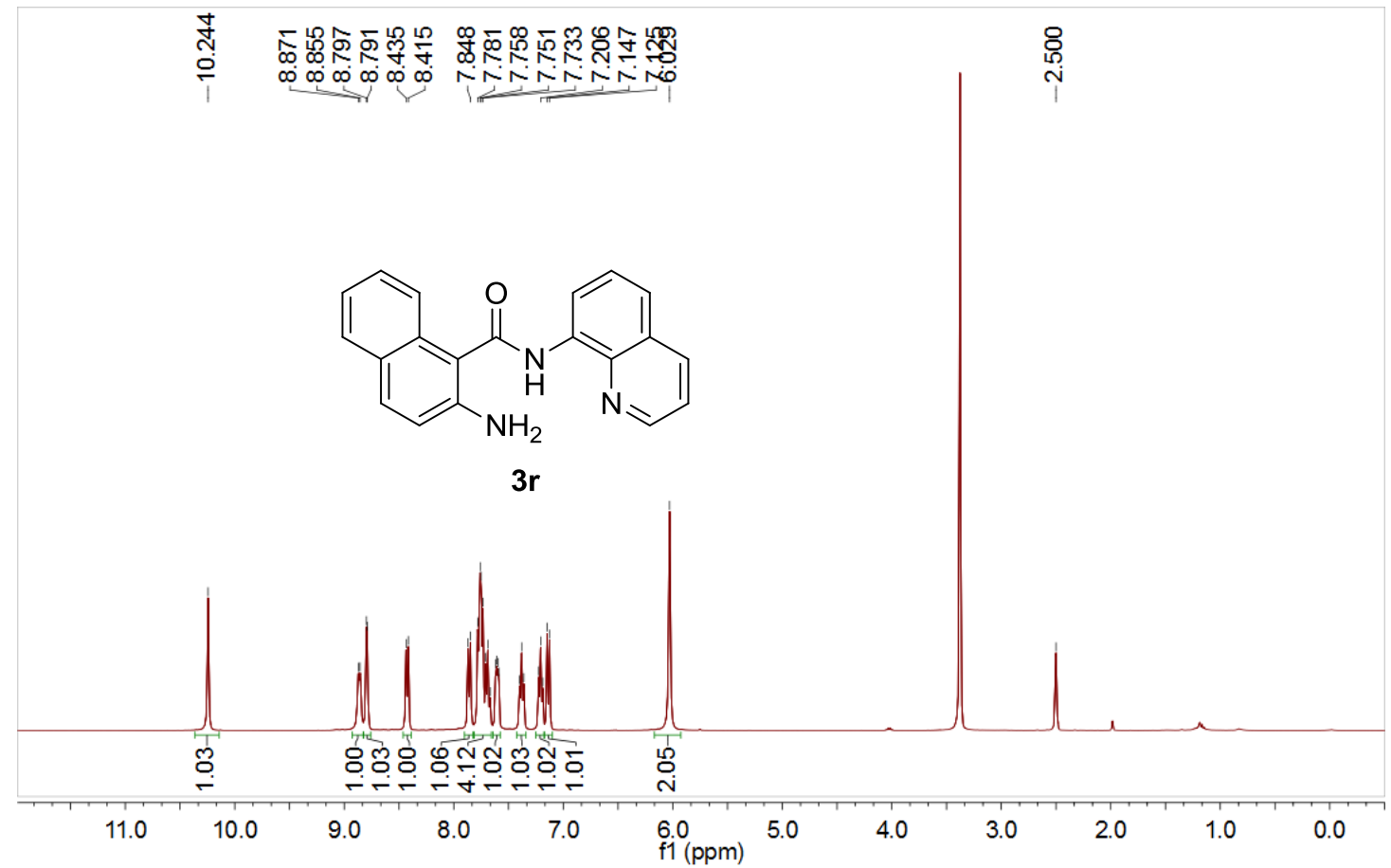


${ }^{13}$ C NMR Spectrum of $\mathbf{3 r}$

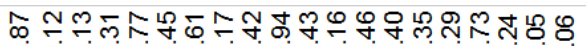

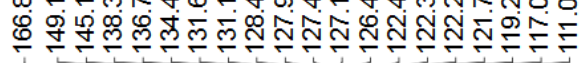

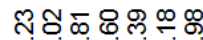

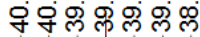

$\overbrace{\mathrm{NH}_{2}}^{(}$

$3 \mathbf{r}$

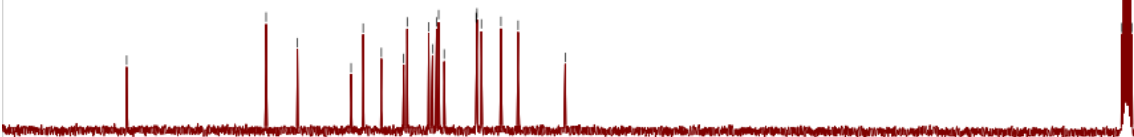

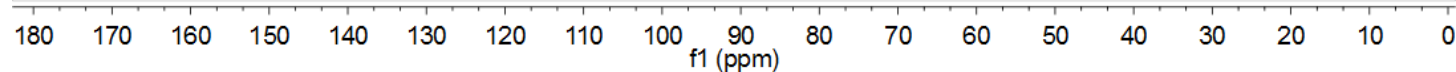

${ }^{1} \mathrm{H}$ NMR Spectrum of $\mathbf{3 s}$

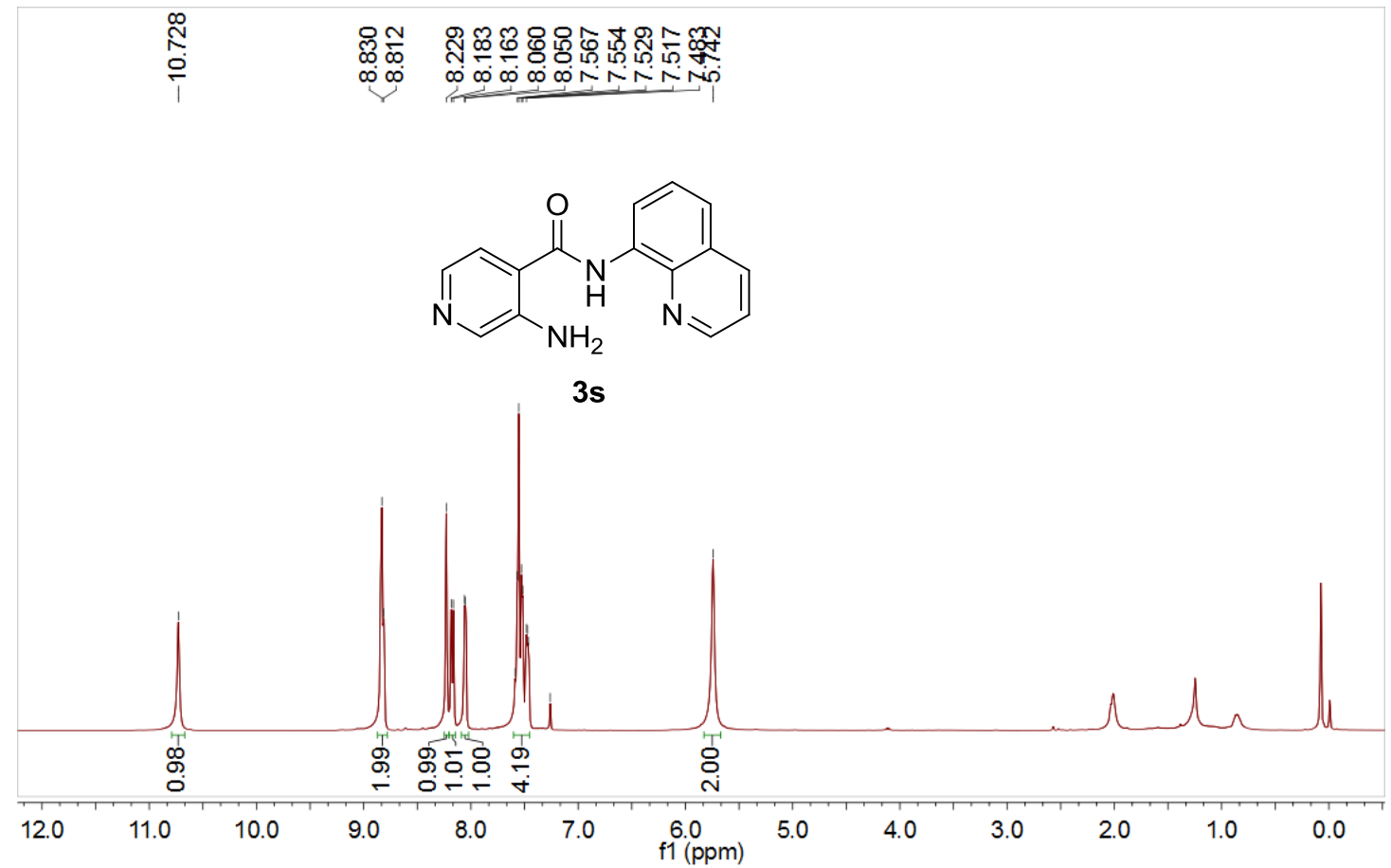


${ }^{13} \mathrm{C}$ NMR Spectrum of $3 \mathrm{~s}$

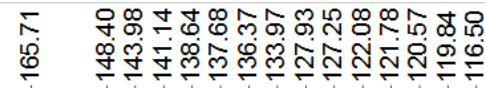

이요

쇼인<smiles>Nc1cnccc1C(=O)Nc1cccc2cccnc12</smiles>

3s

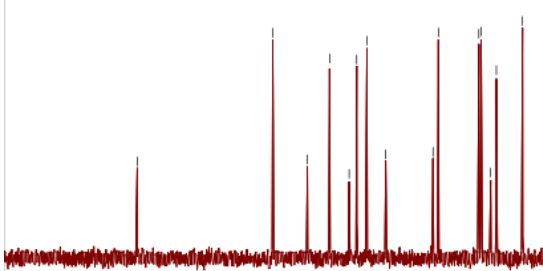

180

$\begin{array}{lllll}170 & 160 & 150 & 140 & 130\end{array}$

120110

$100 \underset{\mathrm{f} 1(\mathrm{ppm})}{90}$

$70 \quad 60 \quad 50$

$40 \quad 30$

$20 \quad 10 \quad 0$

${ }^{1} \mathrm{H}$ NMR Spectrum of $\mathbf{3 t}$

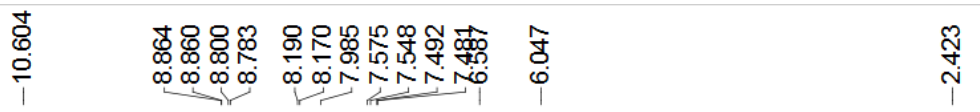

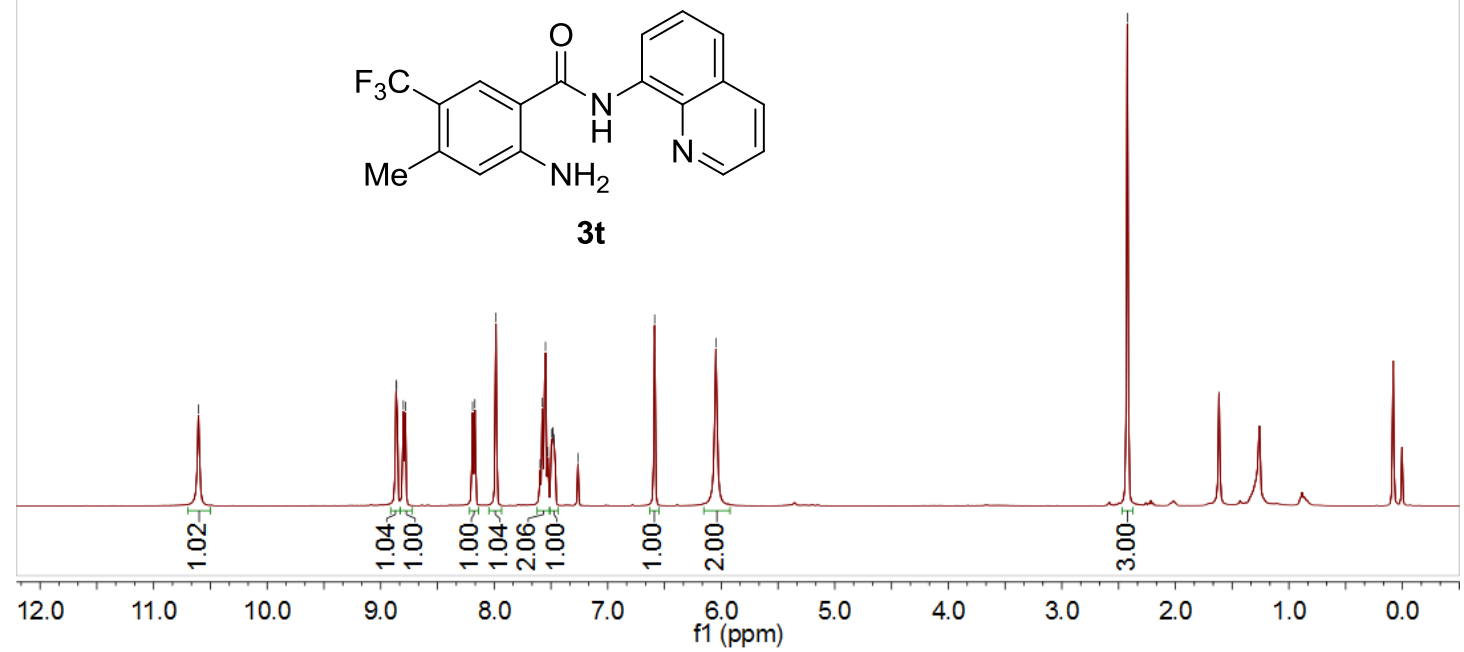

3t 
${ }^{13} \mathrm{C}$ NMR Spectrum of $\mathbf{3 t}$

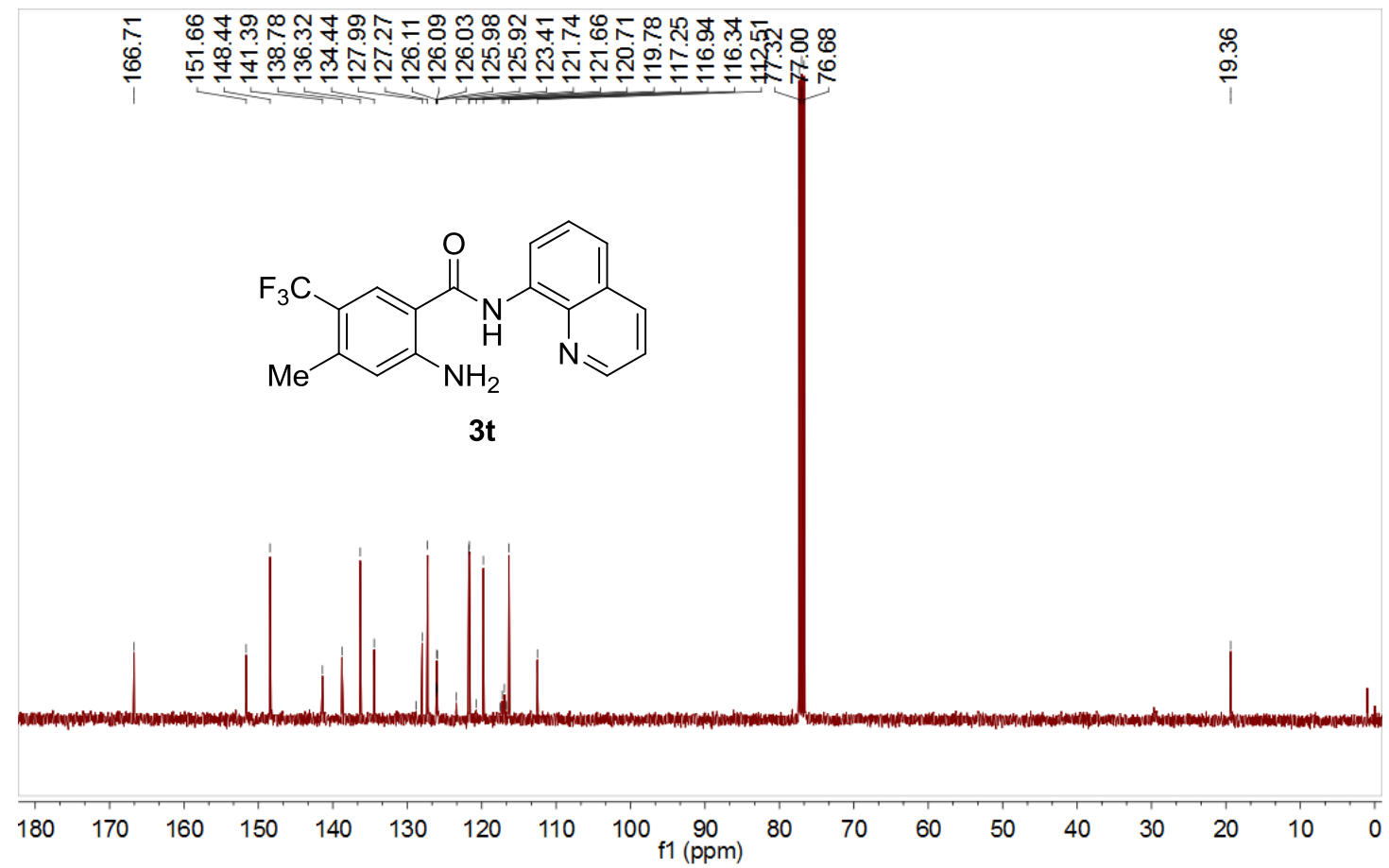

${ }^{19}$ F NMR Spectrum of $\mathbf{3 t}$

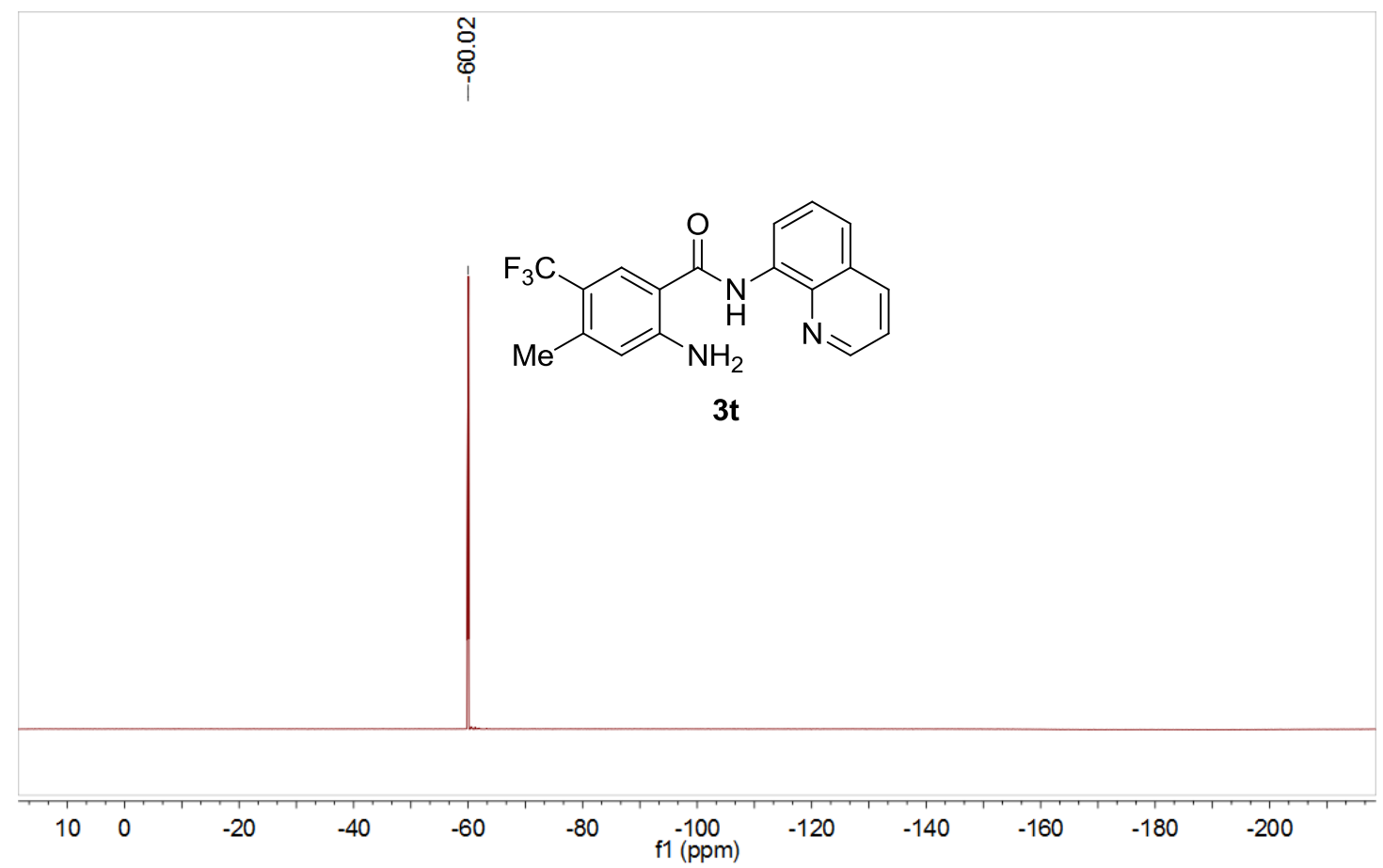


${ }^{1} \mathrm{H}$ NMR Spectrum of $\mathbf{3 u}$

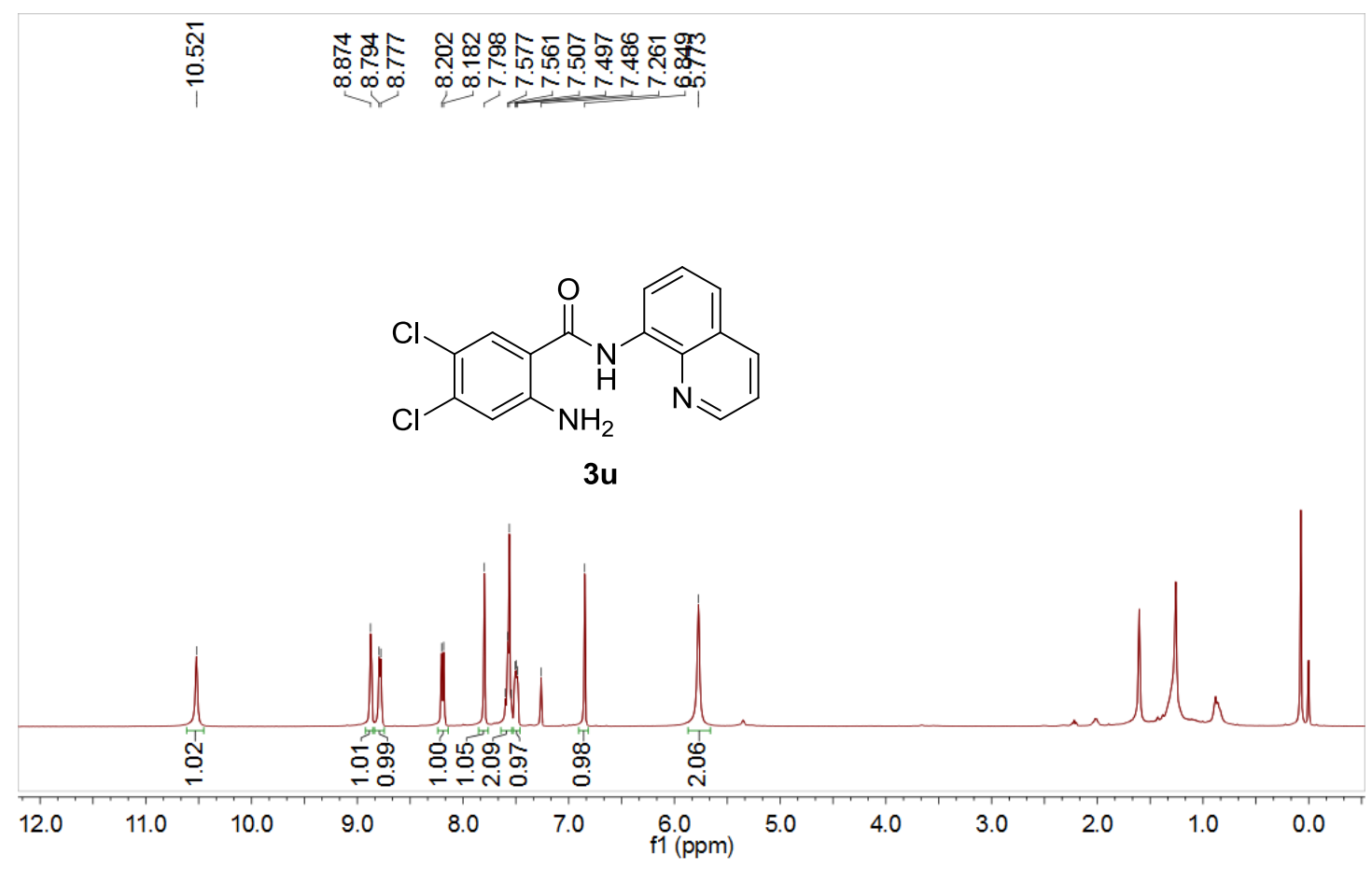

${ }^{13} \mathrm{C}$ NMR Spectrum of $\mathbf{3 u}$

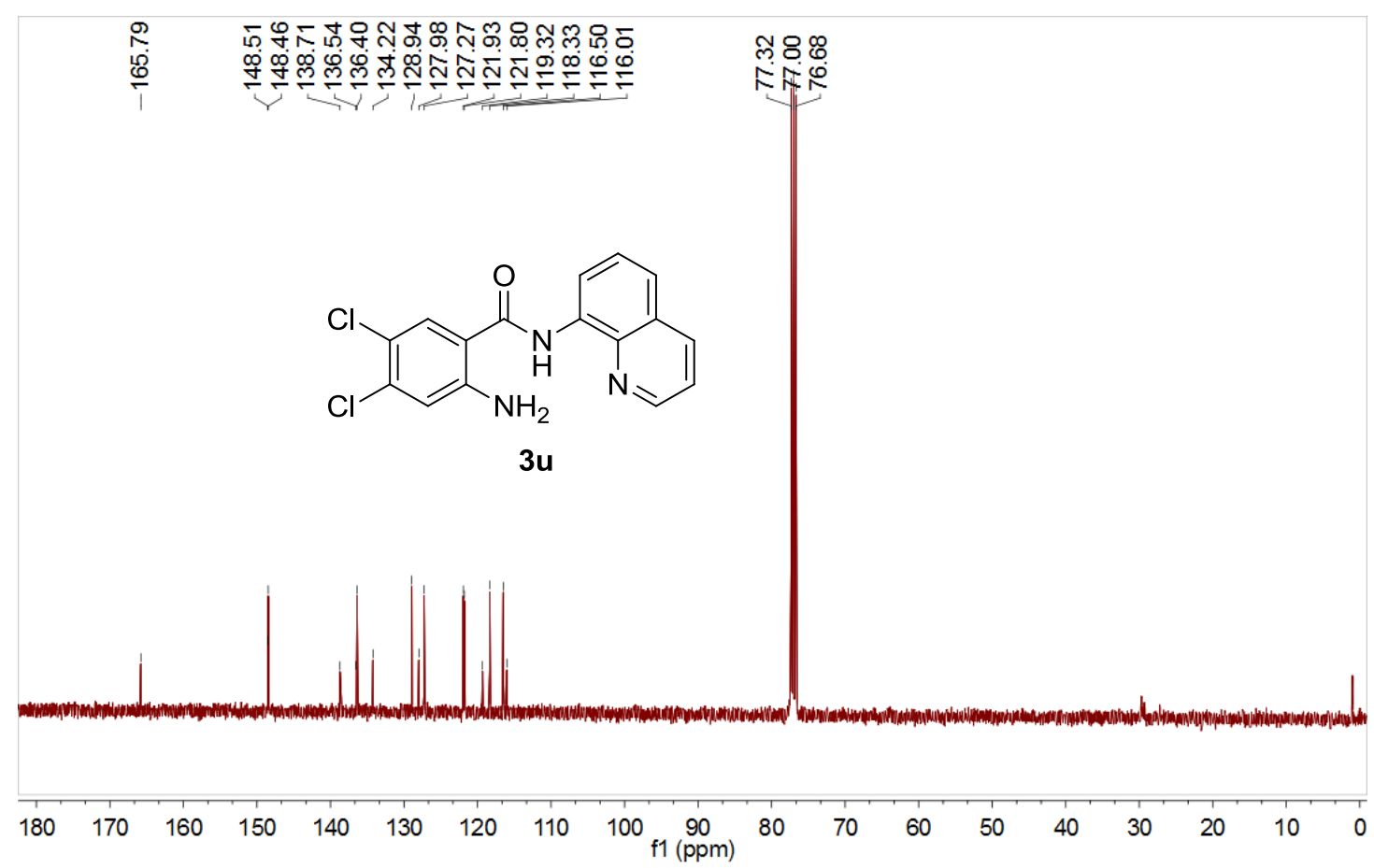


${ }^{1} \mathrm{H}$ NMR Spectrum of $\mathbf{3 w}$

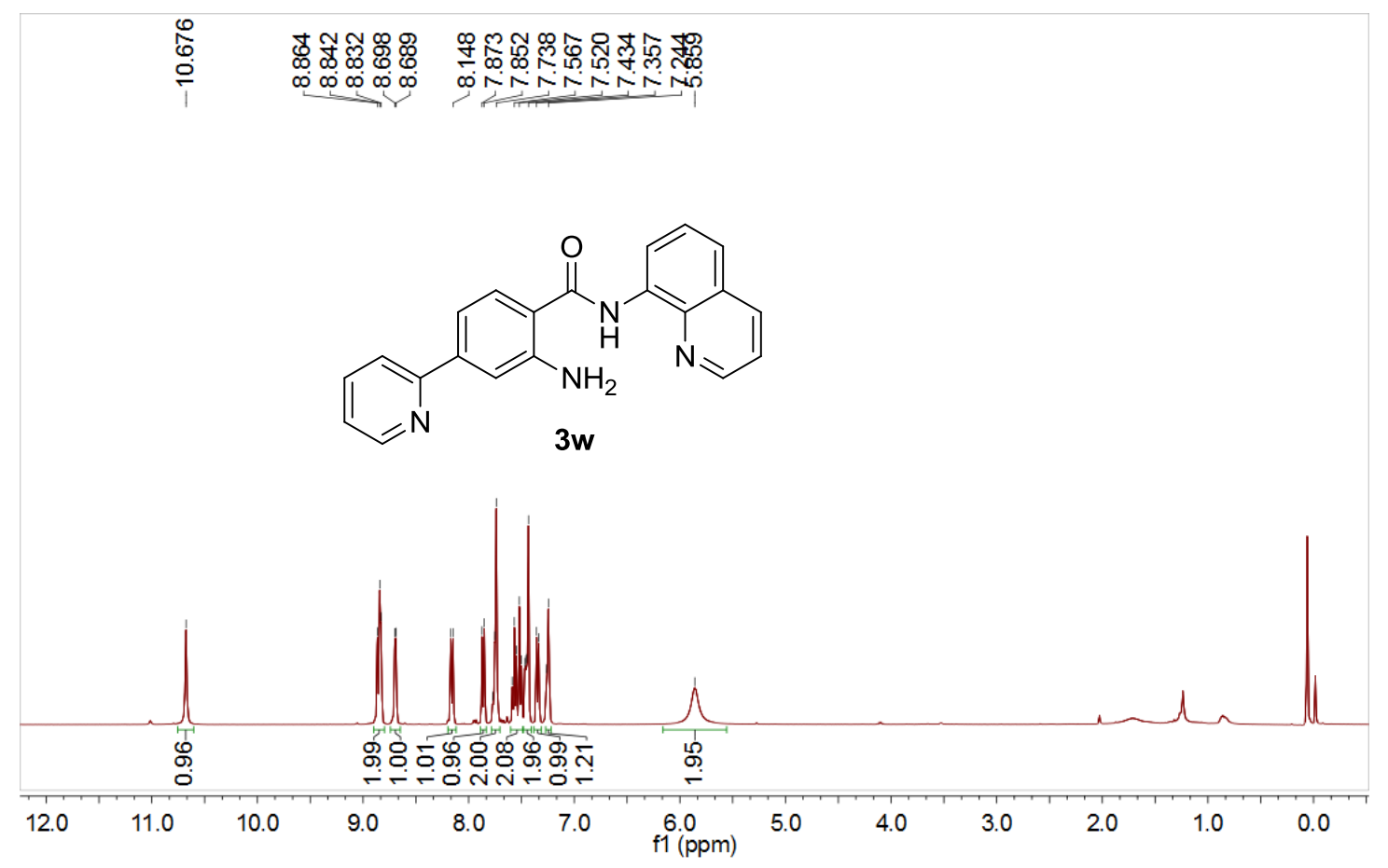

${ }^{13} \mathrm{C}$ NMR Spectrum of $\mathbf{3 w}$

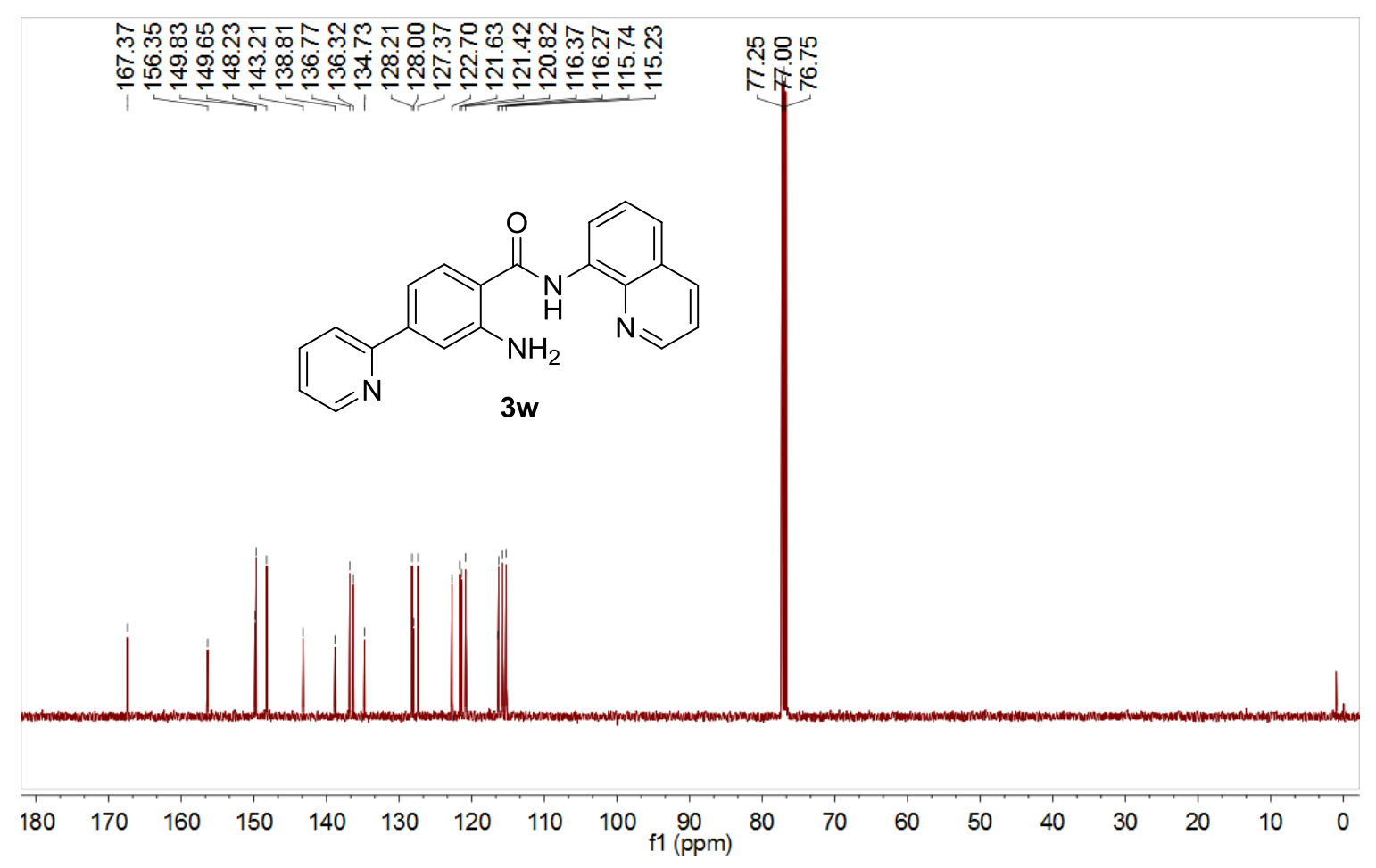


${ }^{1} \mathrm{H}$ NMR Spectrum of $\mathbf{3 x}$

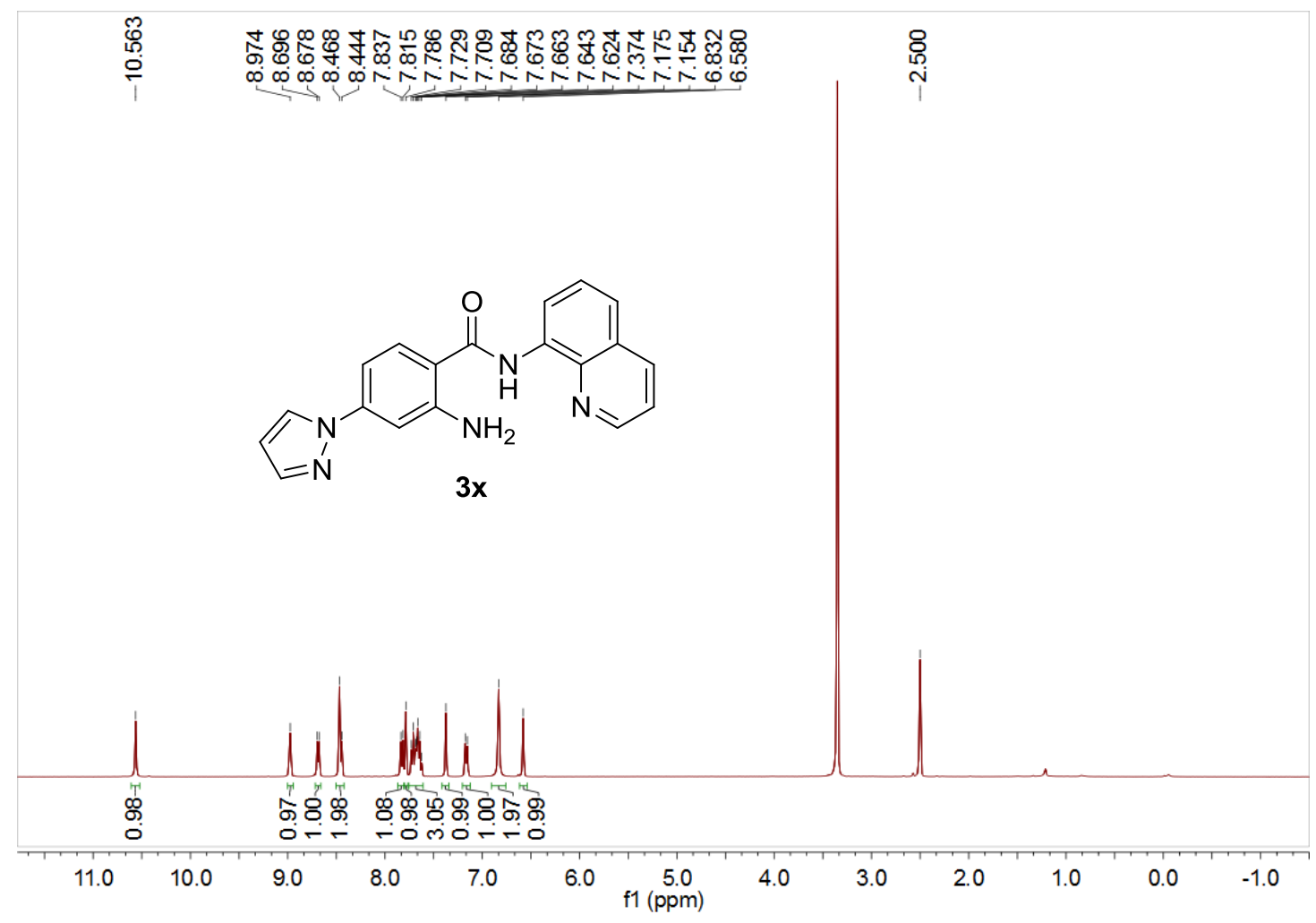

${ }^{13} \mathrm{C}$ NMR Spectrum of $\mathbf{3 x}$

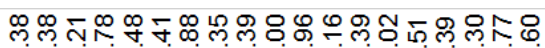

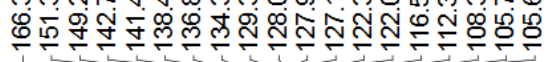

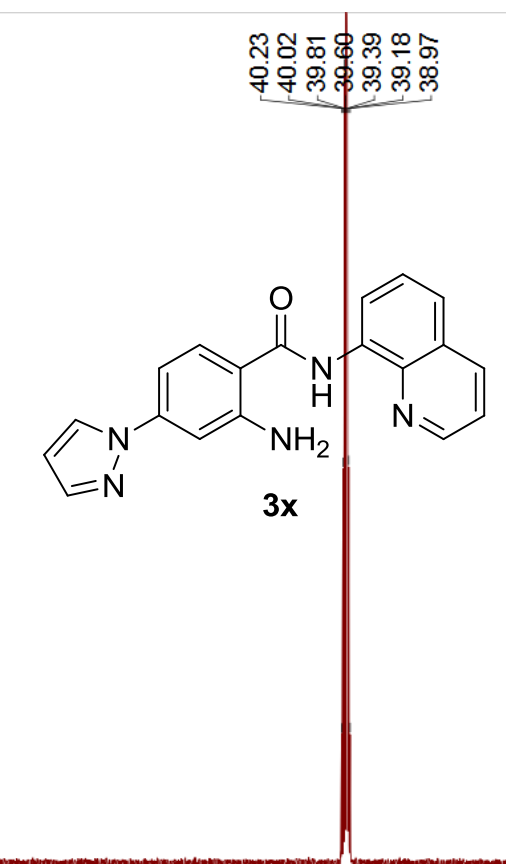

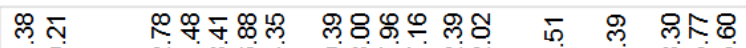

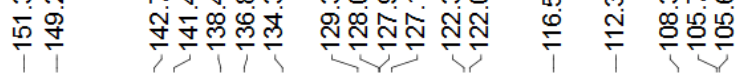

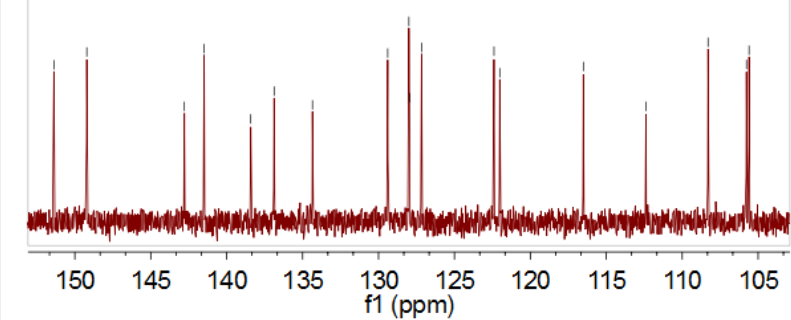

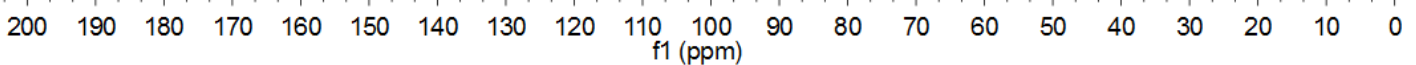

